\title{
TWO-DIMENSIONAL CORE-COLLAPSE SUPERNOVA SIMULATIONS WITH THE ISOTROPIC DIFFUSION SOURCE APPROXIMATION FOR NEUTRINO TRANSPORT
}

\author{
Kuo-Chuan Pan (潘國全), Matthias Liebendörfer, Matthias Hempel, and Friedrich-Karl ThielemanN \\ Physik Department, Universität Basel, Klingelbergstrasse 82, CH-4056 Basel, Switzerland; kuo-chuan.pan@unibas.ch \\ Received 2015 May 11; accepted 2015 November 29; published 2016 January 22
}

\section{ABSTRACT}

The neutrino mechanism of core-collapse supernova is investigated via non-relativistic, two-dimensional (2D), neutrino radiation-hydrodynamic simulations. For the transport of electron flavor neutrinos, we use the interaction rates defined by Bruenn and the isotropic diffusion source approximation (IDSA) scheme, which decomposes the transported particles into trapped-particle and streaming-particle components. Heavy neutrinos are described by a leakage scheme. Unlike the "ray-by-ray" approach in some other multidimensional supernova models, we use cylindrical coordinates and solve the trapped-particle component in multiple dimensions, improving the protoneutron star resolution and the neutrino transport in angular and temporal directions. We provide an IDSA verification by performing one-dimensional (1D) and 2D simulations with 15 and $20 M_{\odot}$ progenitors from Woosley et al. and discuss the difference between our IDSA results and those existing in the literature. Additionally, we perform Newtonian 1D and 2D simulations from prebounce core collapse to several hundred milliseconds postbounce with $11,15,21$, and $27 M_{\odot}$ progenitors from Woosley et al. with the HS(DD2) equation of state. General-relativistic effects are neglected. We obtain robust explosions with diagnostic energies $E_{\text {dia }}$ $\gtrsim 0.1-0.5 \mathrm{~B}\left(1 \mathrm{~B} \equiv 10^{51} \mathrm{erg}\right)$ for all considered $2 \mathrm{D}$ models within approximately $100-300 \mathrm{~ms}$ after bounce and find that explosions are mostly dominated by the neutrino-driven convection, although standing accretion shock instabilities are observed as well. We also find that the level of electron deleptonization during collapse dramatically affects the postbounce evolution, e.g., the neglect of neutrino-electron scattering during collapse will lead to a stronger explosion.

Key words: hydrodynamics - instabilities - neutrinos - supernovae: general

\section{INTRODUCTION}

Nearly five decades after the first attempt to obtain a neutrino-driven explosion by Colgate \& White (1966), the explosion mechanism of core-collapse supernovae (CCSNe) remains elusive. It is generally believed that neutrino transport and convection are important ingredients in achieving a successful explosion (see recent reviews in Janka 2012; Burrows 2013; Foglizzo et al. 2015). However, modeling CCSNe with Boltzmann transport in three dimensions is numerically expensive and too time-consuming with current computing resources, since the neutrino radiation hydrodynamics with Boltzmann transport is essentially a sevendimensional problem: three spatial coordinates, two angular degrees of freedom, neutrino energy, and time. In addition, there are three types of neutrino species and their antiparticles that would require a solution of the Boltzmann equation.

CCSN simulations with Boltzmann transport have been studied in one-dimensional (1D; Mezzacappa \& Bruenn 1993a; Liebendörfer et al. 2001b, 2004; Sumiyoshi et al. 2005), in two-dimensional (2D; Livne et al. 2004; Ott et al. 2008; Brandt et al. 2011), and recently in three-dimensional (3D) with low resolutions and fixed background profiles (Sumiyoshi et al. 2015). However, these 2D and 3D works have ignored the velocity-dependent terms and decoupled these from the energy groups for simplicity, and the spatial resolutions are not sufficient to achieve a correct turbulence cascade. Therefore, approximate methods for the neutrino transport in multidimensional simulations are still necessary at this moment.

Simple approximatation schemes include the light-bulb scheme (Murphy \& Burrows 2008; Hanke et al. 2012; Couch 2013), neutrino leakage (Janka \& Mueller 1996;
Rosswog \& Liebendörfer 2003; O'Connor \& Ott 2011; Couch \& O'Connor 2014), and gray transport (Fryer et al. 1999; Scheck et al. 2006). In these schemes, neutrino transport is relatively cheap, and therefore it is possible to perform highresolution simulations in three dimensions (with effective angular resolutions $\lesssim 1^{\circ}$ ), better describing the turbulence and convection behind the shock and the standing accretion shock instabilities (SASI, Blondin et al. 2003). However the neutrino transport in these schemes is possibly oversimplified because it does not really follow the transport of the neutrino distributions and therefore cannot describe the neutrino heating selfconsistently.

A more sophisticated but still approximated scheme for neutrino transport is called the Moment scheme: the multigroup flux-limited diffusion scheme (Bruenn et al. 2013; Dolence et al. 2015) takes the zeroth angular moment of neutrino moment expansions (i.e., energy density). The M1 moment scheme additionally evolves the momentum density and considers the higher moment closure with an analytic form (Pons et al. 2000; Kuroda et al. 2012; O'Connor \& Ott 2013; Obergaulinger et al. 2014; O'Connor 2015; Kuroda et al. 2015) or by a variable Eddington tensor (Burrows et al. 2000; Rampp \& Janka 2002; Thompson et al. 2003; Buras et al. 2006; Müller et al. 2012b; Melson et al. 2015b). However, as reported by Kuroda et al. (2015), the M1 scheme in 3D general relativity (GR) is still very expensive and difficult to run in long-term postbounce simulations with high resolution (an effective angular resolution $\lesssim 4^{\circ}$ ).

Another approximated transport scheme is the isotropic diffusion source approximation (IDSA, Liebendörfer et al. 2009). In the IDSA, the distribution function is separated into an opaque trapped-particle component and a transparent 
streaming-particle component. The two components are linked by a source term. Therefore, the transport equation becomes a diffusion problem in the opaque region and a ray-tracing problem in the transparent region (Liebendörfer et al. 2009). Multi-dimensional simulations with the IDSA have been performed by Suwa et al. $(2011,2014)$ in 2D and Takiwaki et al. $(2014,2012)$ in 3D. However, most of these multidimensional studies with the IDSA or with other transport schemes do not really solve the neutrino transport in multiple dimensions. Instead, they consider the angular distribution to be several 1D problems, i.e., apply the ray-by-ray ( $\mathrm{RbR})$ approach. Sumiyoshi et al. (2015) and Dolence et al. (2015) have pointed out that the $\mathrm{RbR}$ approach may artificially exaggerate the neutrino flux variations in the angular and temporal components, since the temporal variation of the neutrino fluxes in convective regions is mosttly ignored in the $\mathrm{RbR}$ approach. Additionally, the RbR approximation overestimates the heating of accretion luminosity on its own ray and underestimates the heating in neighboring rays.

In this paper, we present two-dimensional CCSN simulations with the IDSA for neutrino transport in cylindrical coordinates, which are in principle easy to extend to 3D and have better resolution and boundary conditions for the description of the proto-neutron star (PNS) than simulations in spherical coordinates. We solve the trapped-particle component in multiple dimensions to improve the neutrino transport in angular and temporal directions. Furthermore, we study the effects of neutrino transport during collapse by comparing our IDSA solver with a parameterized deleptonization scheme from Liebendörfer (2005). We find that the postbounce explosion dynamics is sensitive to the detailed neutrino interactions before core bounce, such as neutrino-electron scattering (NES) and electron-capture rates. Additional effects such as GR and magnetic fields are also crucial factors in studying the CCSN explosion mechanism, in particular for fast-rotating and more massive progenitors, but we leave these parts for future work. The numerical methods and the IDSA implementations are described in the following section. A verification of our IDSA implementation and a comparison with other neutrino transport schemes are shown in Section 3. In Section 4, we apply our IDSA implementation to multiple progenitors, using a new SN equation of state (EOS). In Section 5, we summarize our results and conclude. A discussion of the different EOSs is presented in the Appendix.

\section{NUMERICAL METHODS AND MODELS}

We describe the numerical code and the corresponding setup of our simulations in Section 2.1. The method and implementation of the IDSA for neutrino transport are demonstrated in Section 2.2. We present the investigated EOS and supernova progenitors in Sections 2.3 and 2.4.

\subsection{Numerical Code and Initial Setup}

We use FLASH ${ }^{1}$ version 4 (Fryxell et al. 2000; Dubey et al. 2008; Lee 2013) to solve the Eulerian equations of hydrodynamics,

$$
\frac{\partial \rho}{\partial t}+\nabla \cdot(\rho v)=0
$$

\footnotetext{
http://flash.uchicago.edu
}

Table 1

Neutrino Interactions Included in the Simulations

\begin{tabular}{lc}
\hline \hline Neutrino Interaction & Reference \\
\hline 1. $\nu_{e}+n \rightleftharpoons e^{-}+p$ & Bruenn (1985) \\
2. $\bar{\nu}_{e}+p \rightleftharpoons e^{+}+n$ & Bruenn (1985) \\
3. $\nu_{e}+A \rightleftharpoons e^{-}+A^{\prime}$ & Bruenn (1985) \\
4. $\nu+\alpha / A / N \rightleftharpoons \nu+\alpha / A / N$ & Bruenn (1985) \\
5. $\nu+e^{ \pm} \rightleftharpoons \nu+e^{ \pm}$ & Mezzacappa \& Bruenn (1993b), Lie- \\
& bendörfer (2005) \\
\hline
\end{tabular}

Notes. $n=$ free neutrons, $p=$ free protons, $N=$ free neutrons or protons, $A=$ nuclei besides $\alpha$ particles, $\alpha=$ alpha particles, $\nu_{e}=$ electron-type neutrinos, $\bar{\nu}_{e}=$ electron-type antineutrinos, $\nu=$ all types of neutrinos, $e^{-}=$electron, and $e^{+}=$positron.

${ }^{\mathrm{a}}$ In this paper, we include the neutrino-electron scattering by using the parameterized deleptonization in Liebendörfer (2005) in the prebounce phase, since this reaction is only important during core collapse.

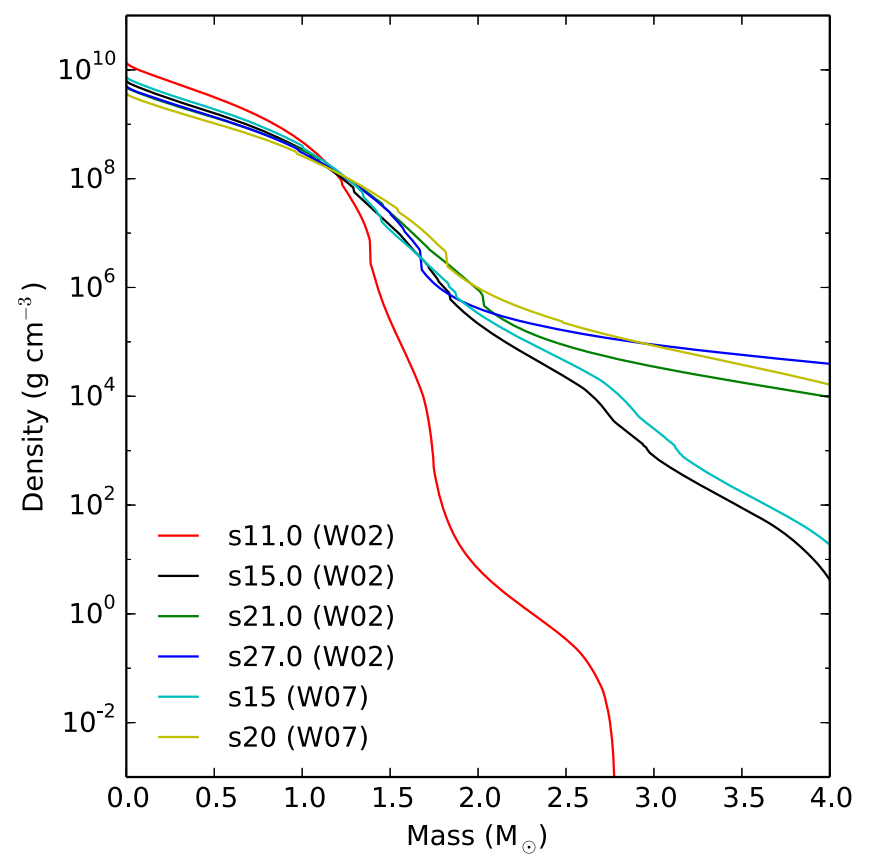

Figure 1. Density as a function of enclosed mass for the four investigated progenitor models from Woosley et al. (2002) and Woosley \& Heger (2007).

$$
\begin{gathered}
\frac{\partial \rho \boldsymbol{v}}{\partial t}+\nabla \cdot(\rho \boldsymbol{v} \boldsymbol{v})+\nabla P=-\rho \nabla \Phi \\
\frac{\partial \rho E}{\partial t}+\nabla \cdot[(\rho E+P) \boldsymbol{v}]=-\rho \boldsymbol{v} \cdot \nabla \Phi \\
\frac{\partial \rho Y_{e}}{\partial t}+\nabla \cdot\left(\rho \boldsymbol{v} Y_{e}\right)=0 \\
\frac{\partial \rho Y_{l}^{t}}{\partial t}+\nabla \cdot\left(\rho \boldsymbol{v} Y_{l}^{t}\right)=0 \\
\frac{\partial\left(\rho Z_{l}^{t}\right)^{3 / 4}}{\partial t}+\nabla \cdot\left(\boldsymbol{v}\left(\rho Z_{l}^{t}\right)^{3 / 4}\right)=0,
\end{gathered}
$$

where $\rho$ is the gas density, $v$ is the velocity vector, $P$ is the gas pressure, $E$ is the total specific energy, $\Phi$ is the gravitational potential, $Y_{e}$ is the electron fraction, $Y^{t}$ is the particle number fraction, and $Z$ is the particle mean specific energy. The index $l$ labels different species of trapped particles (i.e., $\nu_{e}$ and $\overline{\nu_{e}}$ ). A 
Table 2

Parameters for the Parameterized Deleptonization

\begin{tabular}{llllll}
\hline \hline Progenitor & $\mathrm{s} 11.0(\mathrm{~W} 02)$ & $\mathrm{s} 15.0(\mathrm{~W} 02)$ & $\mathrm{s} 21.0(\mathrm{~W} 02)$ & $\mathrm{s} 27.0(\mathrm{~W} 02)$ & $\mathrm{s} 15(\mathrm{~W} 07)$ \\
\hline$\rho_{1}\left(\mathrm{~g} \mathrm{~cm}^{-3}\right)$ & $1.5 \times 10^{8}$ & $9.0 \times 10^{7}$ & $2.5 \times 10^{8}$ & $2.5 \times 10^{8}$ & $2.2 \times 10^{8}$ \\
$\rho_{2}\left(\mathrm{~g} \mathrm{~cm}^{-3}\right)$ & $1.2 \times 10^{13}$ & $9.0 \times 10^{12}$ & $1.0 \times 10^{13}$ & $1.0 \times 10^{13}$ & $3.5 \times 10^{12}$ \\
$Y_{1}$ & 0.5 & 0.5 & 0.5 & 0.5 & 0.5 \\
$Y_{2}$ & 0.287 & 0.282 & 0.279 & 0.279 & $0.0 \times 10^{8}$ \\
$Y_{c}$ & 0.02 & 0.03 & 0.017 & 0.017 & 0.279 \\
\hline
\end{tabular}

Note. Parameters used in the fitting formula from Liebendörfer (2005).
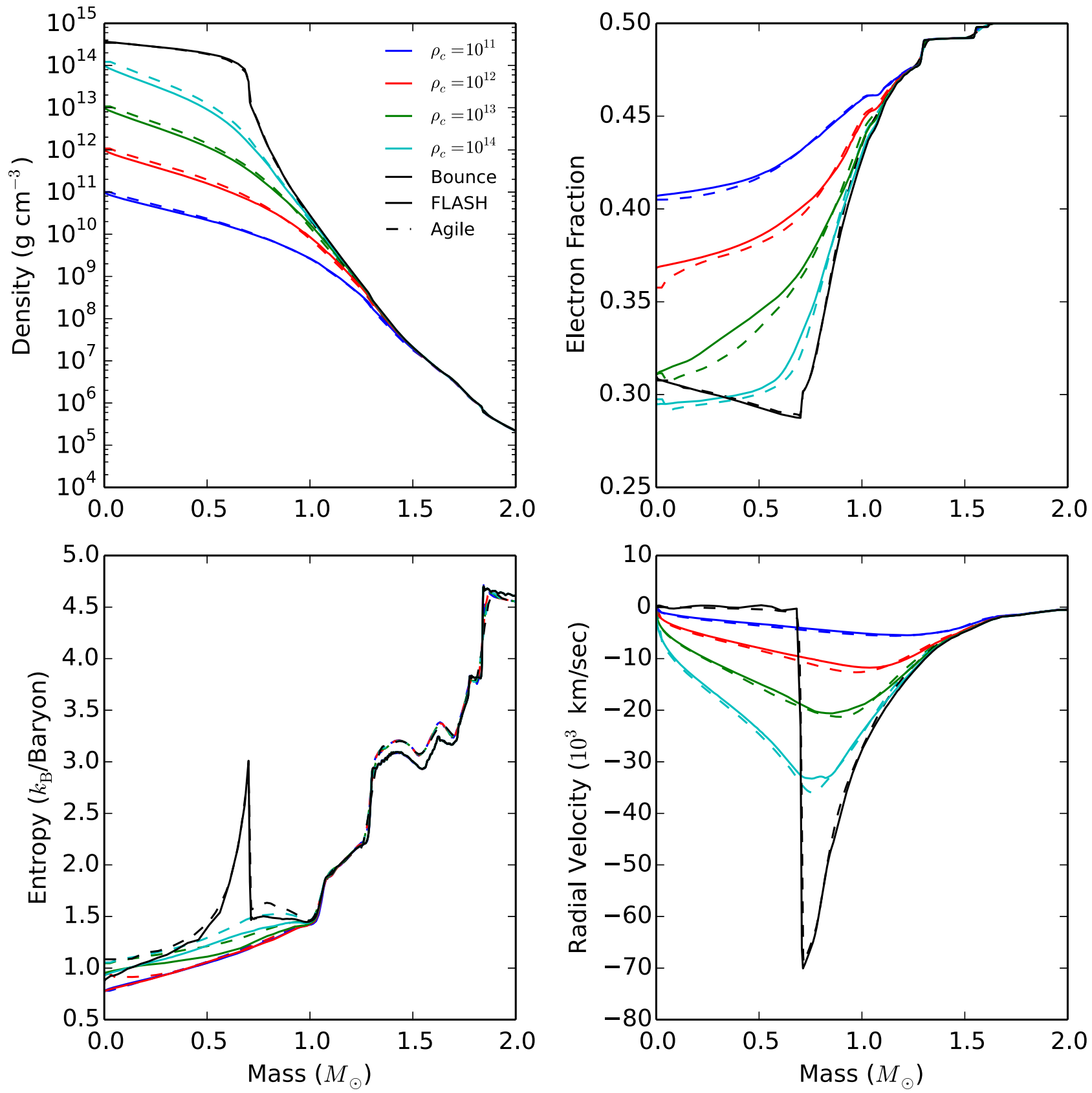

Figure 2. 1D radial profiles in the mass coordinate during the collapse of the s15.0 progenitor with LS220 EOS. Different colors represent different times when the central density reaches $\rho_{c}=10^{11}, 10^{12}, 10^{13}$, and $10^{14} \mathrm{~g} \mathrm{~cm}^{-3}$ and at bounce. Solid lines show simulation results from FLASH and dashed lines show simulation results from AGILE-IDSA. Note that there are some slight time offsets between the results from FLASH and AGILE-IDSA because their output files do not exactly match the given central densities. 


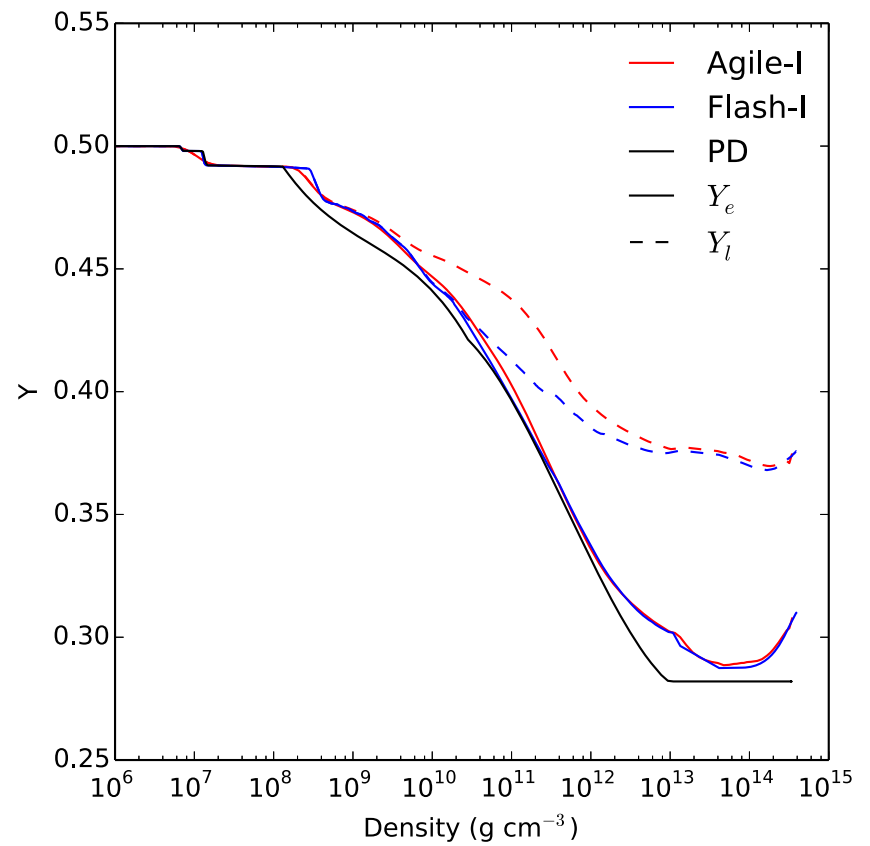

Figure 3. Profiles of electron fraction (solid lines) and lepton fraction (dashed lines) at core bounce in model s15.0. Red lines show simulation data from Agile-IDSA and blue lines represent data from FLASH. The black line indicates the electron fraction from the fitted PD scheme (Table 2). Both Agile-IDSA and FLASH are 1D simulations with LS220 EOS and without NES.

detailed description of the neutrino transport method will be presented in Section 2.2.

FLASH is a parallel, multidimensional hydrodynamics code based on block-structured adaptive mesh refinement (AMR). Our simulation setup is essentially similar to what has been implemented in Couch (2013) and Couch \& O'Connor (2014), but replaces their radiation transfer solver by an IDSA solver (Liebendörfer et al. 2009). We use the third-order piecewise parabolic method (PPM, Colella \& Woodward 1984) for spatial reconstruction, the HLLC Riemann solver and the Hybrid slope limiter for shock-capture. The "hybrid" Riemann solver is widely used to avoid an odd-even numerical instability when the shock is aligned with the grid (Quirk 1991). However, we do not see this instability in our CCSN simulations by comparing simulation results with the hybrid Riemann solver. The HLLC Riemann solver shows a better turbulent cascade based on the implicit large-eddy simulations (Radice et al. 2015). Effects from general and special relativity and from magnetic fields are ignored.

Simulations are performed in 1D spherical and 2D cylindrical coordinates. The center of a progenitor star is located at the origin of the simulation box. The simulation box includes the inner $10^{4} \mathrm{~km}$ in radius of a progenitor star in $1 \mathrm{D}$ and the full $180^{\circ}$ in cylindrical coordinates with $r_{\text {max }}=z_{\max }=10^{4} \mathrm{~km}$ and $z_{\min }=-10^{4} \mathrm{~km}$ in $2 \mathrm{D}$. The central $r \lesssim 100 \mathrm{~km}$ sphere has the smallest zone size of $0.488 \mathrm{~km}$ and the AMR mesh is dynamically adjusted based on the magnitude of the second derivatives of gas density, pressure, and entropy. To save computation time, we apply additional AMR decrements based on the distance to the origin. For example, the first AMR decrement is enforced at $r \sim 100 \mathrm{~km}$ and the second at $r \sim 200 \mathrm{~km}$, the next at $r \sim 400 \mathrm{~km}$, and so on. The maximum zone size is $62.5 \mathrm{~km}$. We employ the "outflow" boundary condition at the outer boundaries and the "reflect" boundary condition at the inner boundaries. The gravitational potential is solved by the new improved multipole solver in FLASH (Couch et al. 2013) with a maximum Legendre order, $l_{\max }=16$.

It should be noted that the "outflow" boundary condition, as reported by Couch (2013), causes a zero-gradient mass accretion at the boundary which will overestimate the inflow and suppress the explosion at late time. We therefore extend our simulation domain to $10,000 \mathrm{~km}$ to minimize the effect of boundary conditions.

\subsection{Neutrino Transport}

In the IDSA (Liebendörfer et al. 2009; Berninger et al. 2013), we decompose the distribution function $f$ of transported particle species and the neutrino transport operator $D$ into a trapped-particle component and a streaming-particle component. We assume that these two components evolve separately. With this assumption, we rewrite the transport equation, $D\left(f=f^{t}+f^{s}\right)=C$, where $C=C^{t}+C^{s}$ is a collision integral, by two equations,

$$
\begin{gathered}
D\left(f^{t}\right)=C^{t}-\Sigma \\
D\left(f^{s}\right)=C^{s}+\Sigma,
\end{gathered}
$$

where $\Sigma$ is the diffusion source term, which converts trapped particles $(t)$ into streaming particles $(s)$ and vice versa.

By using the diffusion limit, the diffusion source term $\Sigma$ in the trapped transport equation could be expressed as (see Liebendörfer et al. 2009 for a more detailed discussion)

$$
\Sigma=\min \left\{\max \left[\alpha+(j+\chi) \frac{1}{2} \int f^{s} d \mu, 0\right], j\right\},
$$

where

$$
\alpha=\nabla \cdot\left(\frac{-1}{3(j+\chi+\phi)} \nabla f^{t}\right)
$$

is a non-local diffusion scalar, $j$ is the emissivity, $\chi$ is the absorptivity, $\phi$ is the opacity, and $\mu$ is the angle cosine. The distribution functions $f^{t}$ and $f^{s}$ can be solved by using Equations (8) and (10) in Liebendörfer et al. (2009). Once the distribution functions are known, the net interaction rates of transported particles can be calculated and further updates of the fluid quantities, such as $\dot{v}, \dot{Y}_{e}, \dot{E}, \dot{Y}_{l}^{t}$, and $\dot{Z}_{l}^{t}$, can be derived.

We solve the streaming component in $1 \mathrm{D}$ with the original IDSA solver, but solve the trapped component and $\alpha$ in multiple dimensions. In each time step, we take the angleaveraged radial bins of fluid and neutrino quantities, such as $\rho$, $s, T, Y_{e}, Y_{l}^{t}$, and $Z_{l}^{t}$, as $1 \mathrm{D}$ inputs for the streaming component. The radial bins contain 600 zones sampled from the center of the PNS, defined by the location of maximum density, up to $r=5000 \mathrm{~km}$. The radial bins are uniformly spaced $(\Delta r=2 \mathrm{~km})$ up to a radius of $r=400 \mathrm{~km}$. Beyond $400 \mathrm{~km}$, the zone spacing increases logarithmically. The streaming component carries only to the location of neutrino spheres $R_{\nu}(E)$ and the angular integration of the spectral neutrino flux $F_{s}(E)$. The local heating from streaming neutrinos is then determined based on the local neutrino interaction rates, which in turn are based on the multidimensional hydrodynamic quantities. 

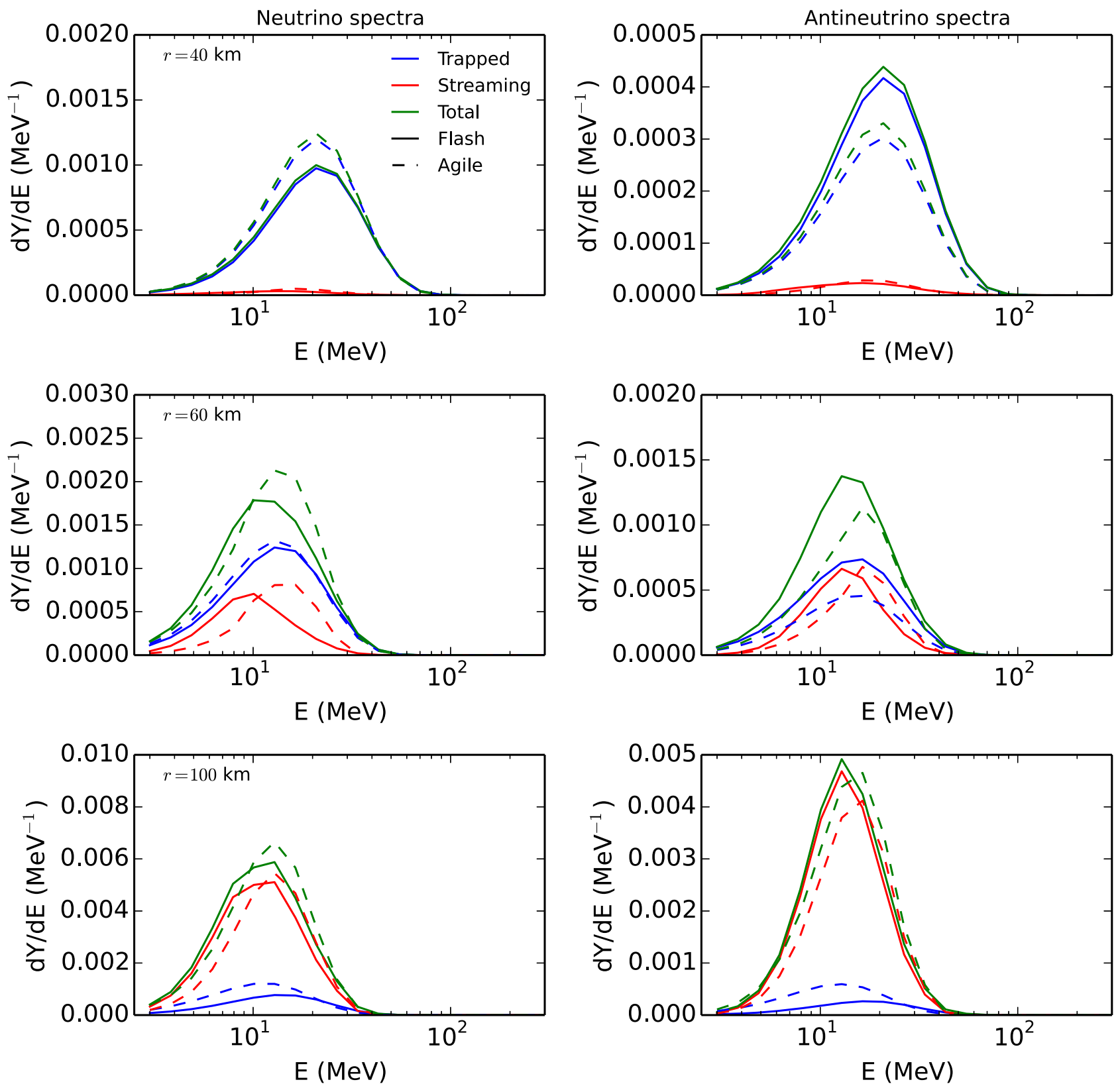

Figure 4. Particle spectra at $150 \mathrm{~ms}$ postbounce for the trapped-particle component (blue lines), streaming-particle component (red lines), and their sum (green lines). Solid lines show spectra from FLASH and dashed lines represent spectra from AGILE-IDSA. Left panels show neutrino spectra and right panels show antineutrino spectra. Each row indicates spectra at a different radius.

It should be noted that the assumption of spherical symmetry in the streaming component may calculate the neutrino flux and heating incorrectly when shocks become highly asymmetric, especially in 2D simulations after the SASI have developed. On the other hand, an RbR approach may artificially enhance the asymmetry and lead to incorrect results as well (Dolence et al. 2015; Sumiyoshi et al. 2015). It is important to have multidimensional simulations with both approximations because they are likely to bracket the actual solution: the RbR focuses all heating from the accretion luminosity on its own ray, while the angular integration distributes it over all directions. In reality, a solution in between these extremes is expected.

For the trapped component, we explicitly solve the diffusion source $\Sigma$ and $\alpha$ in multiple dimensions and update $f^{t}$ locally. Together with the streaming component, the new multidimensional interaction rates can be updated. The new neutrino sources are re-evaluated based on multidimensional neutrino sources and production rates, and then used for the next streaming step. Since we solve the diffusion part explicitly, a stable solution requires a small neutrino time step $t_{\nu} \sim \Delta x^{2} /(2 \lambda c) \sim \Delta x / 2 c$. Therefore, in our 1D and 2D simulations, we use a fixed hydrodynamic time step with $t_{\text {hydro }}=10^{-6} \mathrm{~s}$ and do sub-cycling for the neutrino transport. Since the IDSA solver in the streaming component allows a larger time step, we adopt two types of sub-cycles: one for the streaming component with $t_{\nu}^{s}=5 \times 10^{-7} \mathrm{~s}$ and the other for the trapped component with $t_{\nu}^{t}=10^{-7} \mathrm{~s}$. Additionally, the neutrino pressure contributes extra momentum and can be 

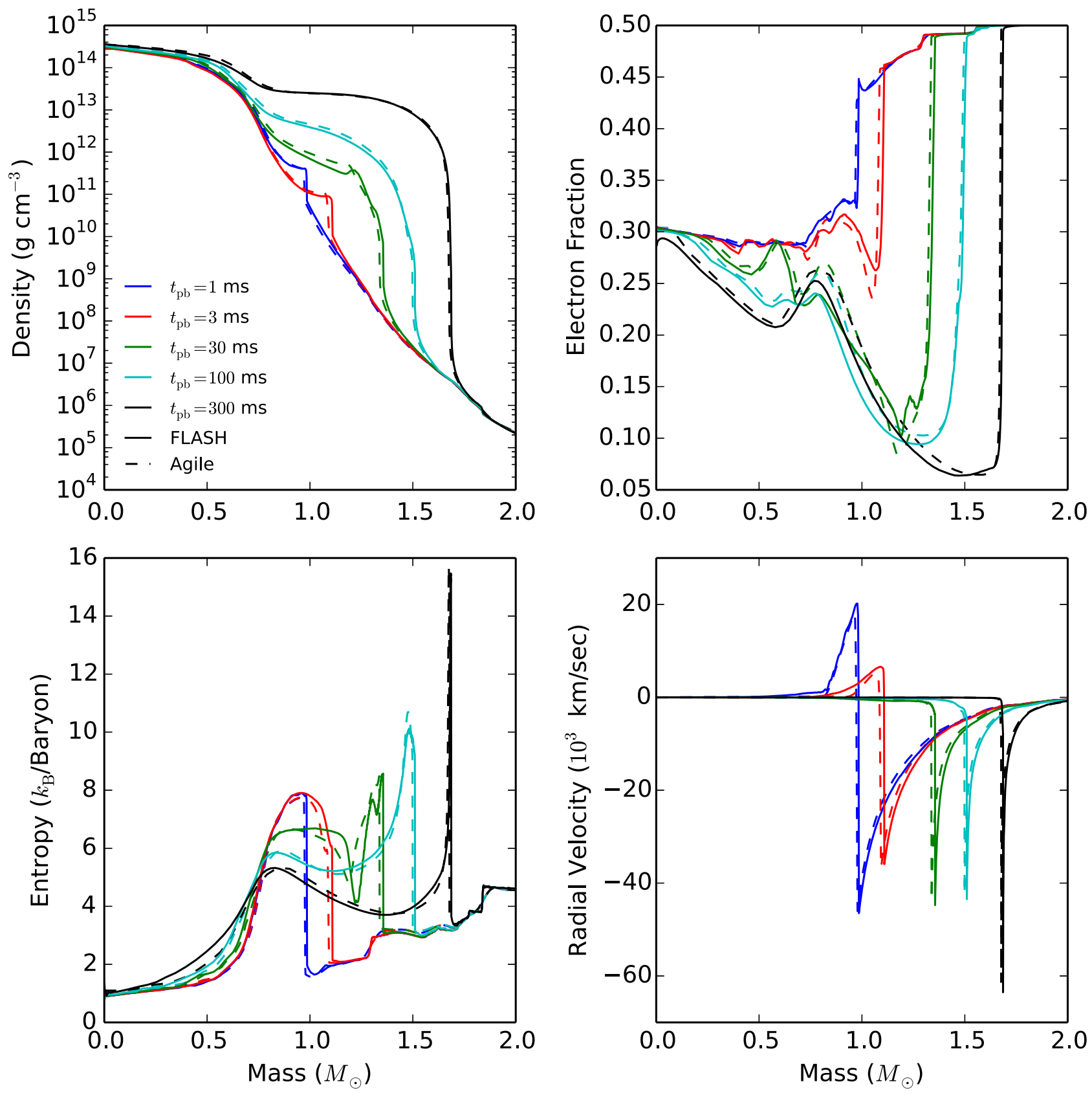

Figure 5. Similar to Figure 2, but for the postbounce evolution.

expressed by

$$
\frac{\partial \boldsymbol{v}}{\partial t}=-\frac{1}{\rho} \nabla\left(\frac{\rho Z_{l}^{t}}{3 m_{\mathrm{b}}}\right) .
$$

We note that Equations (6) and (11) include all thermodynamically important $O(v / c)$ terms of the Boltzmann transport equation. These $O(v / c)$ terms have been considered as crucial for CCSN modeling (Mezzacappa \& Bruenn 1993a; Liebendörfer et al. 2009; Lentz et al. 2012b).

Our IDSA solver only includes electron flavor neutrinos. We use 20 energy bins that are spaced logarithmically from 3 to $300 \mathrm{MeV}$. For heavy neutrinos, the energy release is treated by a leakage scheme that is based on the local diffusion and the local production rates (Hannestad \& Raffelt 1998; Rosswog \& Liebendörfer 2003; Perego et al. 2014).

Table 1 summarizes all weak interactions that are included in this work. All weak interactions in the IDSA solver use the Bruenn description (Bruenn 1985). Note that the IDSA solver dose not include NES (interaction 5 in Table 1). NES is important during the collapse phase but provides minor contributions in the postbounce phase (Bruenn 1989a; see Section 3.2 for a demonstration). Liebendörfer (2005) found a simple relation between the electron deleptonization and the gas density in the collapse phase, where electron fraction and entropy can be parameterized by density and chemical potentials. This parameterized deleptonization (PD) scheme can effectively take interactions into account that are only implemented in the Boltzmann solver that is used to determine 


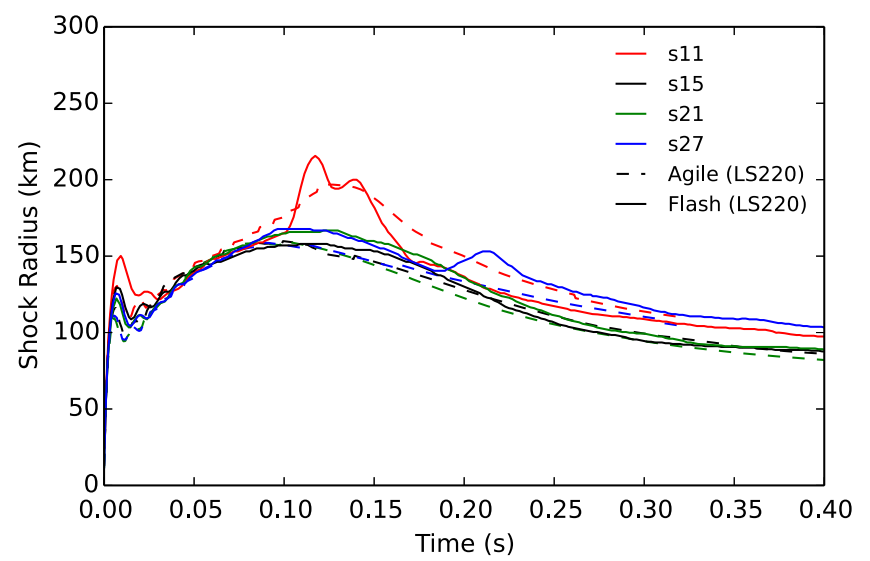

Figure 6. Time evolution of the shock radius for the four investigated progenitor models in both FLASH (solid lines) and AGILE-IDSA (dashed lines). Different colors represent different progenitor models. All results are 1D with LS220 EOS and without NES. The differences in the models s11.0 and s27.0 after $0.1 \mathrm{~s}$ are due to a different handling of shell interfaces and a large diffusivity of the implicit adaptive mesh in AGILE-IDSA.

the parameters for the PD scheme. We have recalibrated the fitting parameters with different progenitors for the PD scheme by running AGILE-BOLTZTRAN (Liebendörfer et al. 2004), since the original parameters in Liebendörfer (2005) are calibrated for the progenitors G15 (s15s7b2, Woosley \& Weaver 1995) and N13 (Nomoto \& Hashimoto 1988). Table 2 summarizes the fitting parameters we use during collapse. In this paper we include the effect of NES by using the PD scheme in the collapse phase. Since the scheme is independent of the neutrino fractions, we update the neutrino fractions and energy through the IDSA solver during collapse. After bounce, we turn off the PD and switch to the IDSA solver. For simulations without NES, we use the IDSA solver from the beginning of core collapse to postbounce.

To verify our IDSA implementation in FLASH, we provide a code comparison of FLASH-IDSA with AGILE-IDSA and with existing results from the literature in Section 3.

\subsection{Equation of State}

We use the nuclear EOS unit in FLASH, which incorporates the finite-temperature EOS routines from O'Connor \& Ott (2010) and Couch (2013). ${ }^{2}$ The Lattimer \& Swesty EOS (with incompressibility, $K=220 \mathrm{MeV}$; Lattimer \& Swesty 1991) and Hempel \& Schaffner-Bielich (HS) DD2 EOS are used in this work. The HS(DD2) EOS employs the density-dependent relativistic mean-field interactions of Typel et al. (2010). The description of nuclei in supernova matter is based on Hempel \& Schaffner-Bielich (2010). This EoS was first applied in corecollapse supernova simulations by Fischer et al. (2014), where further details can be found. LS220 is one of the most common and well-studied EOSs in the supernova community. However, it has some deficiencies, for example it is based on the singlenucleus approximation for heavy nuclei, and considers only the alpha particle as a degree of freedom of all possible light nuclei. See Hempel et al. (2015) for a comparison of predictions for cluster formation for the HS(DD2) and the LS220 EOSs with experiments, where good agreement was found. Furthermore, it was shown by Krüger et al. (2013) and

\footnotetext{
http://www.stellarcollapse.org
}

Fischer et al. (2014) that the neutron-matter EOS of LS220 is in disagreement with constraints from chiral effective field theory. Furthermore, its low-density symmetry energy deviates from constraints obtained from finite nuclei, see Figure 9 of Hempel (2015). No multidimensional simulations have been performed with HS(DD2) at this moment. We use LS220 for a code verification test in Section 3 and then use DD2 for our main simulations in Section 4. A low-density extension for the EOS is included in the routines from O'Connor \& Ott (2010). In the Appendix we provide a brief discussion of the differences between LS220 and DD2.

\subsection{Supernova Progenitors}

In this paper, we consider four different non-rotating, solarmetallicity progenitors, s11.0, s15.0, s21.0, and s27.0 from Woosley et al. $(2002)^{3}$, for our multiple progenitor study. We also consider two non-rotating, solar-metallicity progenitors, s15 and s20 from Woosley \& Heger (2007), for a comparison study. Figure 1 shows the initial density distribution of these six progenitors. The s11.0 progenitor has the highest core density but the most dilute envelope. The s21.0, s27.0, and s20 progenitors have a similar density distribution and the most massive envelope among the models, but have different masses of the iron core and $\mathrm{Si} / \mathrm{O}$ shell. The locations of regions with a high density gradient correspond to the $\mathrm{Si} / \mathrm{O}$ interface. For the same progenitor mass, $\mathrm{s} 15$ has a denser core and more massive envelope than s15.0. Another common progenitor model used in the literature is the $\mathrm{s} 15 \mathrm{~s} 7 \mathrm{~b} 2$ progenitor from Woosley \& Weaver (1995). It has the same progenitor mass as s15.0 and s15 but the $\mathrm{Si} / \mathrm{O}$ interface is located much further inside. This difference may make significant changes to the postbounce evolution of the shock radius when the shock reaches the interface because of a different mass-accretion history (Suwa et al. 2014).

To adopt the progenitor models in FLASH, we map the onedimensional density, temperature, electron fraction, and radial velocity profiles from Woosley et al. (2002) into our 1D/2D grids in FLASH. Other thermodynamic quantities are recalculated using the EOS solver in FLASH. Neutrino fractions are set to zero at the beginning.

\section{IDSA VERIFICATION}

To verify the IDSA implementation in FLASH, we first compare our 1D FLASH simulations with simulations with AGILE-IDSA (Liebendörfer et al. 2009) in Section 3.1. AGILE-IDSA is a 1D spherically symmetric Lagrangian code that is publicly available online. ${ }^{4}$ In Liebendörfer et al. (2009), a nice agreement of AGILE-IDSA with the GR Boltzmann code AGILE-BOLTZTRAN (Liebendörfer et al. 2004) was shown. Since we want to compare our results with the same neutrino transport scheme (IDSA), we run additional simulations in AGILE-IDSA but turn off the GR correction for the gravitational potential. We also turn off the PD during collapse in both codes to test the IDSA solver for neutrino transport before and after bounce.

In Section 3.2, we show 1D and 2D FLASH simulations with the two widely used progenitors, s15 and s20 from Woosley \& Heger (2007), and discuss the differences compared to other

\footnotetext{
http://2sn.org/stellarevolution/

4 https://physik.unibas.ch/ liebend
} 

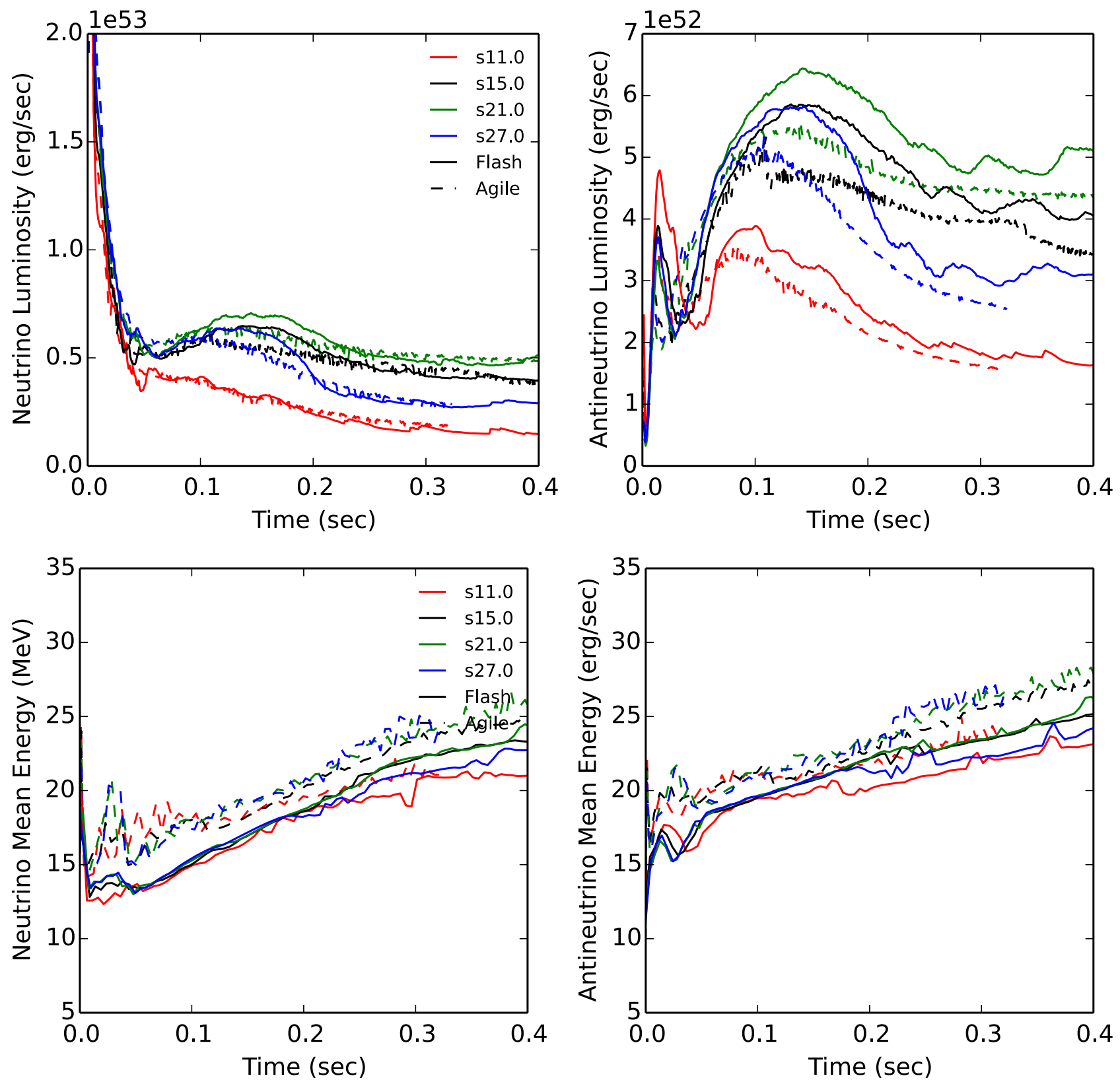

Figure 7. Electron neutrino (left) and electron antineutrino (right) luminosities and mean energies as functions of time. The quantities are averaged over a 5 ms time interval. Different colors represent the different progenitor models. Solid lines represent the FLASH simulations and dashed lines indicate the AGILE-IDSA simulations.

transport schemes. Furthermore, we run AGILE-BOLTZTRAN simulations to demonstrate the importance of NES during core collapse and discuss the influence of the utilized neutrino opacities and electron capture rates on the shock evolution and neutrino signal.

\subsection{Code Comparison with AGILE-IDSA}

We perform 1D CCSN simulations of the four investigated progenitors (s11.0, s15.0, s21.0, and s27.0) with the LS220 EOS in both FLASH and AGILE-IDSA. Figure 2 shows the radial profiles of density, electron fraction, entropy, and radial velocity of the progenitor s15.0 during collapse. It is clear to see that the bounce profiles (black lines in Figure 2) are nearly identical in the two codes. Small differences at the center could originate from the fact that AGILE-IDSA is a Lagrangian code with a moving mesh. At the beginning of a simulation, the innermost region of the progenitor star is much less resolved than with the Eulerian grid of FLASH. Therefore, the radial profiles in AGILE-IDSA evolve slightly more slowly than those in FLASH. Furthermore, the bounce time in FLASH is about 0.1-12 ms later than in AGILE-IDSA, depending on the progenitor star.

Figure 3 shows the electron and lepton fractions of the progenitor s15.0 at core bounce. It is found that the electron fraction is consistent in both codes, but the lepton fraction shows a mismatch at $10^{10} \lesssim \rho \lesssim 10^{12} \mathrm{~g} \mathrm{~cm}^{-3}$, suggesting a lower electron neutrino distribution at low densities in FLASH. However, this difference does not alter the postbounce 

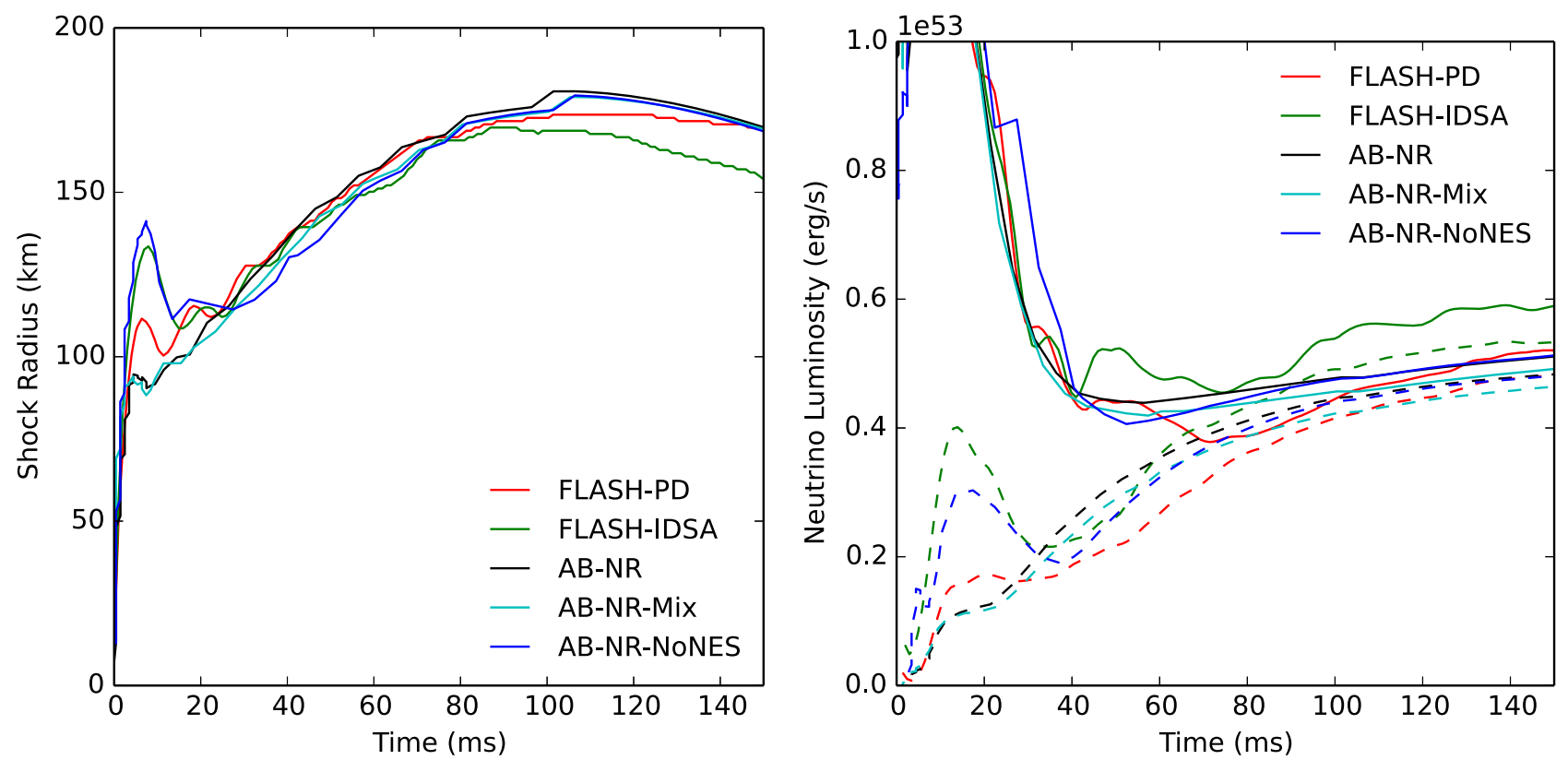

Figure 8. Time evolution of shock radius (left) and electron-type neutrino luminosity (right). Different colors represent different models from FLASH and AGILEBOLTZTRAN with different neutrino physics. See Section 3.2 for a detailed description.
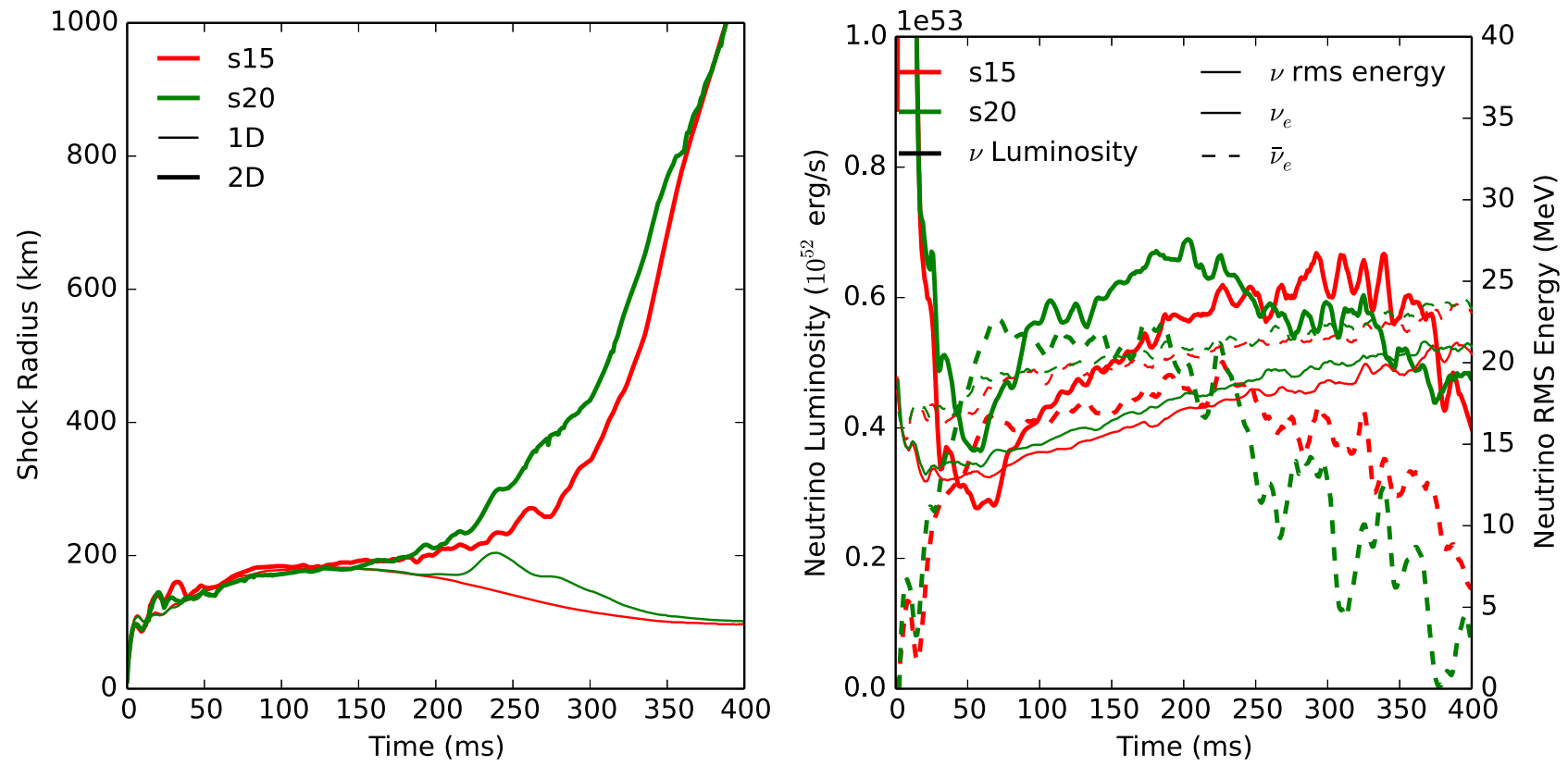

Figure 9. Time evolution of shock radius (left) and electron-type neutrino signals (right). Different colors represent different progenitor models. In the left panel, thick lines show the evolution of 2D simulations and thin lines represent 1D simulations. In the right panel, thick lines indicate electron-type neutrino luminosities and thin lines show the rms neutrino mean energies. Solid lines represent electron neutrino signals and dashed lines represent electron antineutrino signals.

evolution much since the neutrinos are simply free-streaming in the low-density region. We note that in our multidimensional IDSA solver, we have employed a limiter for the diffusion scalar $\alpha$. The limiter enforces the trapped component to have a small value, when $r>1.25 \times R_{\nu}(E)$, to prevent unphysical oscillation in $\alpha$, when the trapped neutrino density goes to zero in the free-streaming regime. This limiter leads to small differences of the neutrino luminosity and mean energy, but does not affect the hydrodynamic quantities. Figure 4 shows the particle spectra of the trapped and streaming components at
$150 \mathrm{~ms}$ postbounce of the progenitor s15.0. We show three different regions where the trapped-particle component dominates (at $r=40 \mathrm{~km}$ ), where trapped- and streaming-particle components are comparable (at $r=60 \mathrm{~km}$ ), and where the streaming-particle component dominates $(r=100 \mathrm{~km})$. There are small differences in the particle spectra, but we do not expect exactly identical spectra in the two codes, since the hydrodynamic parts are different.

A comparison of radial profiles of the density, electron fraction, entropy, and radial velocity of the progenitor s15.0 in 
Table 3

1D Simulation Results During Core Collapse

\begin{tabular}{|c|c|c|c|c|c|c|c|c|}
\hline \multirow[t]{2}{*}{ Property } & \multicolumn{8}{|c|}{ Models } \\
\hline & \multicolumn{2}{|c|}{$\mathrm{s} 11.0$} & \multicolumn{2}{|c|}{$\mathrm{s} 15.0$} & \multicolumn{2}{|c|}{$\mathrm{s} 21.0$} & \multicolumn{2}{|c|}{$\mathrm{s} 27.0$} \\
\hline $\begin{array}{l}\text { Progenitor mass }\left(M_{\odot}\right) \\
\text { Progenitor compactness }\left(\xi_{1.75}\right) \\
\text { Progenitor compactness }\left(\xi_{2.5}\right)\end{array}$ & & & & & & & & \\
\hline Abbreviation (1D-) & $\begin{array}{l}\text { w PD } \\
\text { DP11 }\end{array}$ & $\begin{array}{l}\text { wo PD } \\
\text { DA11 }\end{array}$ & $\begin{array}{l}\text { w PD } \\
\text { DP15 }\end{array}$ & $\begin{array}{l}\text { wo PD } \\
\text { DA15 }\end{array}$ & $\begin{array}{l}\text { w PD } \\
\text { DP21 }\end{array}$ & $\begin{array}{c}\text { wo PD } \\
\text { DA21 }\end{array}$ & $\begin{array}{l}\text { w PD } \\
\text { DP27 }\end{array}$ & $\begin{array}{l}\text { wo PD } \\
\text { DA27 }\end{array}$ \\
\hline Time at $\rho_{c}=10^{11} \mathrm{~g} \mathrm{~cm}^{-3}(\mathrm{~ms})$ & 176.3 & 194.1 & 267.6 & 278.2 & 269.7 & 284.3 & 273.0 & 287.2 \\
\hline Time at $\rho_{c}=10^{12} \mathrm{~g} \mathrm{~cm}^{-3}(\mathrm{~ms})$ & 197.6 & 224.2 & 285.7 & 300.8 & 286.3 & 304.0 & 289.4 & 306.9 \\
\hline Time at $\rho_{c}=10^{13} \mathrm{~g} \mathrm{~cm}^{-3}(\mathrm{~ms})$ & 201.9 & 229.3 & 289.6 & 305.5 & 290.2 & 308.4 & 293.2 & 311.4 \\
\hline Time at $\rho_{c}=10^{14} \mathrm{~g} \mathrm{~cm}^{-3}(\mathrm{~ms})$ & 203.0 & 230.7 & 290.7 & 306.8 & 291.2 & 309.7 & 294.3 & 312.7 \\
\hline Time at bounce $(\mathrm{ms})$ & 203.5 & 231.3 & 291.2 & 307.3 & 291.7 & 310.2 & 294.7 & 313.2 \\
\hline Bounce, central $\rho\left(10^{14} \mathrm{~g} \mathrm{~cm}^{-3}\right)$ & 3.21 & 3.39 & 3.14 & 3.43 & 3.08 & 3.35 & 3.10 & 3.45 \\
\hline Bounce, central $Y_{e}$ & 0.287 & 0.316 & 0.282 & 0.312 & 0.279 & 0.310 & 0.279 & 0.311 \\
\hline Bounce, shock position $\left(M_{\odot}\right)$ & 0.54 & 0.77 & 0.56 & 0.76 & 0.56 & 0.75 & 0.56 & 0.75 \\
\hline
\end{tabular}

Note.

${ }^{a}$ Note that the compactness parameter of the s11.0 progenitor is about 10 times smaller than the others due to a smaller core mass and light envelope.

Table 4

Simulation Parameters and Results

\begin{tabular}{|c|c|c|c|c|c|c|c|c|c|}
\hline Progenitor & Abbreviation $^{\mathrm{a}}$ & $N T_{\text {collapse }}{ }^{\mathrm{b}}$ & EOS & $\begin{array}{c}t_{\text {bounce }}{ }^{c} \\
(\mathrm{~ms})\end{array}$ & $\begin{array}{l}t_{400}{ }^{\mathrm{d}} \\
(\mathrm{ms})\end{array}$ & $\begin{array}{l}t_{\text {end }}{ }^{\mathrm{e}} \\
(\mathrm{ms})\end{array}$ & $\begin{array}{c}E_{\mathrm{dia}}{ }^{\mathrm{f}} \\
\text { (B) }\end{array}$ & $\begin{array}{c}M_{\mathrm{PNS}}^{\mathrm{g}} \\
\left(M_{\odot}\right)\end{array}$ & $\begin{array}{c}R_{\mathrm{PNS}}{ }^{\mathrm{h}} \\
(\mathrm{km})\end{array}$ \\
\hline \multicolumn{10}{|c|}{$1 \mathrm{D}$} \\
\hline s15 (W07) & 1D-LA15-07 & IDSA & LS220 & 237 & $\ldots$ & 763 & $\ldots$ & 1.85 & 29.1 \\
\hline s15 (W07) & 1D-LP15-07 & $\mathrm{PD}$ & LS220 & 249 & $\ldots$ & 523 & $\ldots$ & 1.77 & 36.1 \\
\hline s20 (W07) & 1D-LP20-07 & PD & LS220 & 322 & $\ldots$ & 678 & $\cdots$ & 1.99 & 30.4 \\
\hline s11.0 (W02) & 1D-LA11 & IDSA & LS220 & 206 & $\cdots$ & 794 & $\cdots$ & 1.48 & 27.5 \\
\hline s15.0 (W02) & 1D-LA15 & IDSA & LS220 & 273 & $\cdots$ & 727 & $\cdots$ & 1.84 & 29.5 \\
\hline s21.0 (W02) & 1D-LA21 & IDSA & LS220 & 274 & $\cdots$ & 726 & $\cdots$ & 1.98 & 30.4 \\
\hline s27.0 (W02) & 1D-LA27 & IDSA & LS220 & 283 & $\cdots$ & 717 & $\cdots$ & 1.81 & 31.2 \\
\hline s11.0 (W02) & 1D-DA11 & IDSA & DD2 & 231 & $\ldots$ & 766 & $\ldots$ & 1.47 & 32.2 \\
\hline s15.0 (W02) & 1D-DA15 & IDSA & DD2 & 307 & $\cdots$ & 693 & $\ldots$ & 1.84 & 34.6 \\
\hline s21.0 (W02) & 1D-DA21 & IDSA & DD2 & 310 & $\cdots$ & 604 & $\cdots$ & 1.95 & 37.1 \\
\hline s27.0 (W02) & 1D-DA27 & IDSA & DD2 & 313 & $\cdots$ & 687 & $\cdots$ & 1.81 & 36.1 \\
\hline s11.0 (W02) & 1D-DP11 & PD & DD2 & 203 & $\ldots$ & 761 & $\cdots$ & 1.47 & 33.6 \\
\hline s15.0 (W02) & 1D-DP15 & PD & DD2 & 291 & $\ldots$ & 709 & $\ldots$ & 1.84 & 36.6 \\
\hline s21.0 (W02) & 1D-DP21 & PD & DD2 & 292 & $\ldots$ & 708 & $\ldots$ & 1.98 & 36.6 \\
\hline s27.0 (W02) & 1D-DP27 & PD & DD2 & 295 & $\cdots$ & 705 & $\cdots$ & 1.81 & 37.1 \\
\hline \multicolumn{10}{|c|}{$2 \mathrm{D}$} \\
\hline s15 (W07) & 2D-LP15-07 & PD & LS220 & 249 & 312 & 524 & 0.298 & 1.69 & 37.1 \\
\hline s20 (W07) & 2D-LP20-07 & PD & LS220 & 324 & 284 & 490 & 0.347 & 1.86 & 40.5 \\
\hline s15.0 (W02) & 2D-LA15 & IDSA & LS220 & 274 & 209 & 311 & 0.464 & 1.60 & 46.8 \\
\hline s15.0 (W02) & 2D-LA15low ${ }^{\mathrm{i}}$ & IDSA & LS220 & 274 & 210 & 369 & 0.523 & 1.62 & 43.5 \\
\hline s11.0 (W02) & 2D-DA11 & IDSA & DD2 & 232 & 86 & 374 & 0.821 & 1.31 & 42.3 \\
\hline s15.0 (W02) & 2D-DA15 & IDSA & DD2 & 308 & 186 & 417 & 0.506 & 1.65 & 43.5 \\
\hline s21.0 (W02) & 2D-DA21 & IDSA & DD2 & 311 & 189 & 411 & 0.635 & 1.75 & 44.8 \\
\hline s27.0 (W02) & 2D-DA27 & IDSA & DD2 & 314 & 162 & 429 & 0.248 & 1.66 & 42.3 \\
\hline s11.0 (W02) & 2D-DP11 & PD & DD2 & 204 & 170 & 376 & 0.267 & 1.37 & 47.4 \\
\hline s15.0 (W02) & 2D-DP15 & PD & DD2 & 292 & 275 & 456 & 0.255 & 1.71 & 45.4 \\
\hline s21.0 (W02) & 2D-DP21 & PD & DD2 & 292 & 282 & 484 & 0.417 & 1.82 & 44.8 \\
\hline s27.0 (W02) & 2D-DP27 & PD & DD2 & 295 & 199 & 484 & 0.157 & 1.70 & 43.5 \\
\hline
\end{tabular}

Notes.

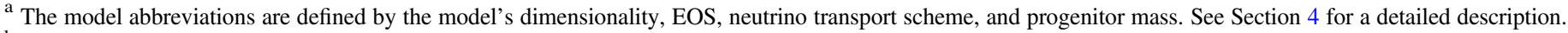

b Neutrino transport scheme during the collapse phase.

c Bounce time.

d Explosion time after bounce.

e Termination time after bounce.

${ }^{\mathrm{f}}$ Diagnostic explosion energy at the end of the simulation. $1 \mathrm{~B} \equiv 10^{51} \mathrm{erg}$.

g PNS mass at the end of the simulation.

${ }^{\mathrm{h}}$ PNS radius (determined as the average radius corresponding to $\rho=10^{11} \mathrm{~g} \mathrm{~cm}^{-3}$ ) at the end of the simulation.

${ }^{\mathrm{i}}$ The effective angular resolution in this model is a factor of two lower. 

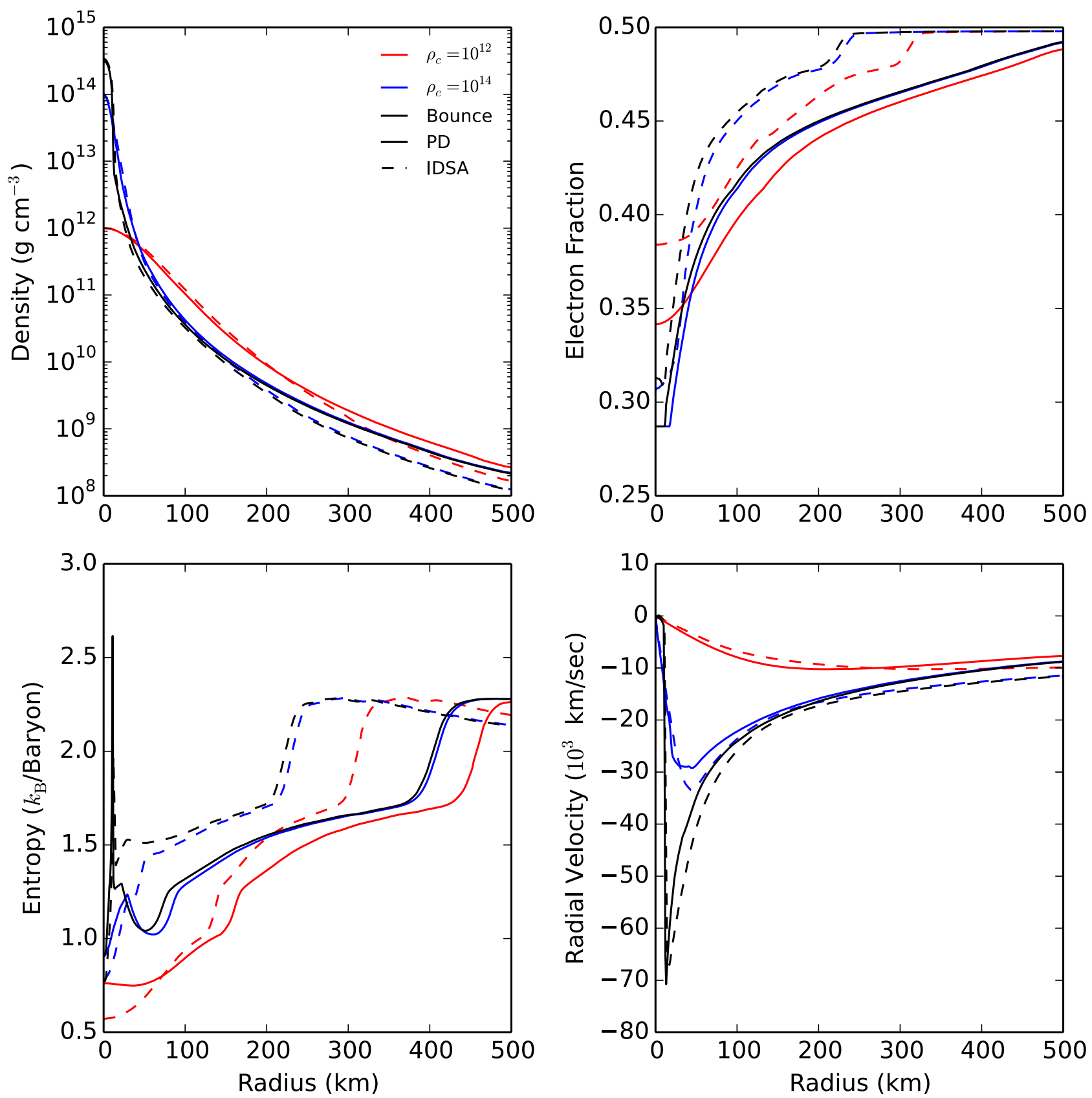

Figure 10. Angle-averaged radial profiles of progenitor s11.0 at different times during core collapse. All models use the DD2 EOS. Different colors indicate different averaged profiles at certain central densities and bounce. The solid lines show simulations with the PD and the dashed lines show simulations with the IDSA.

both codes at different postbounce times is shown in Figure 5. We note that although there are some differences in the radial shock location, the profiles and shock locations are consistent in the mass coordinate. The evolutions of the postbounce shock radius for the four progenitor models are shown in Figure 6. Overall, the evolutions of the shock radius are nearly the same but they show slight differences at $\sim 120 \mathrm{~ms}(\sim 220 \mathrm{~ms})$ in the model s11.0 (s27.0) when the shock reaches the $\mathrm{Si} / \mathrm{O}$ interface.

Figure 7 shows the time evolution of electron-type neutrino luminosity and mean neutrino energy for the four considered models. The values are sampled at a radius of $500 \mathrm{~km}$ in both codes. The electron neutrino luminosities and electron antineutrino luminosities are similar for the two codes around bounce and early postbounce. However, at $\sim 100 \mathrm{~ms}$ postbounce, the neutrino and antineutrino luminosities in FLASH become slightly higher than in AGILE-IDSA for the progenitors s15.0, s21.0, and s27.0. The maximum difference is an increase of $\lesssim 15 \%$ in FLASH at $\sim 150 \mathrm{~ms}$ postbounce. After $\sim 200 \mathrm{~ms}$ postbounce, the neutrino luminosities are back to the same value in both codes but the antineutrino luminosity remains slightly higher in FLASH simulations. For the progenitor s11.0, the electron neutrino luminosity is the same in both codes but the electron antineutrino luminosity is slightly higher in FLASH. However, we note that the mean neutrino and antineutrino energies in FLASH are about $10 \%$ $20 \%$ lower than in AGILE-IDSA. This difference could originate from the lower neutrino fraction in the low-density region in FLASH (see Figure 3), since we measure the neutrino mean energy at $r=500 \mathrm{~km}$. 

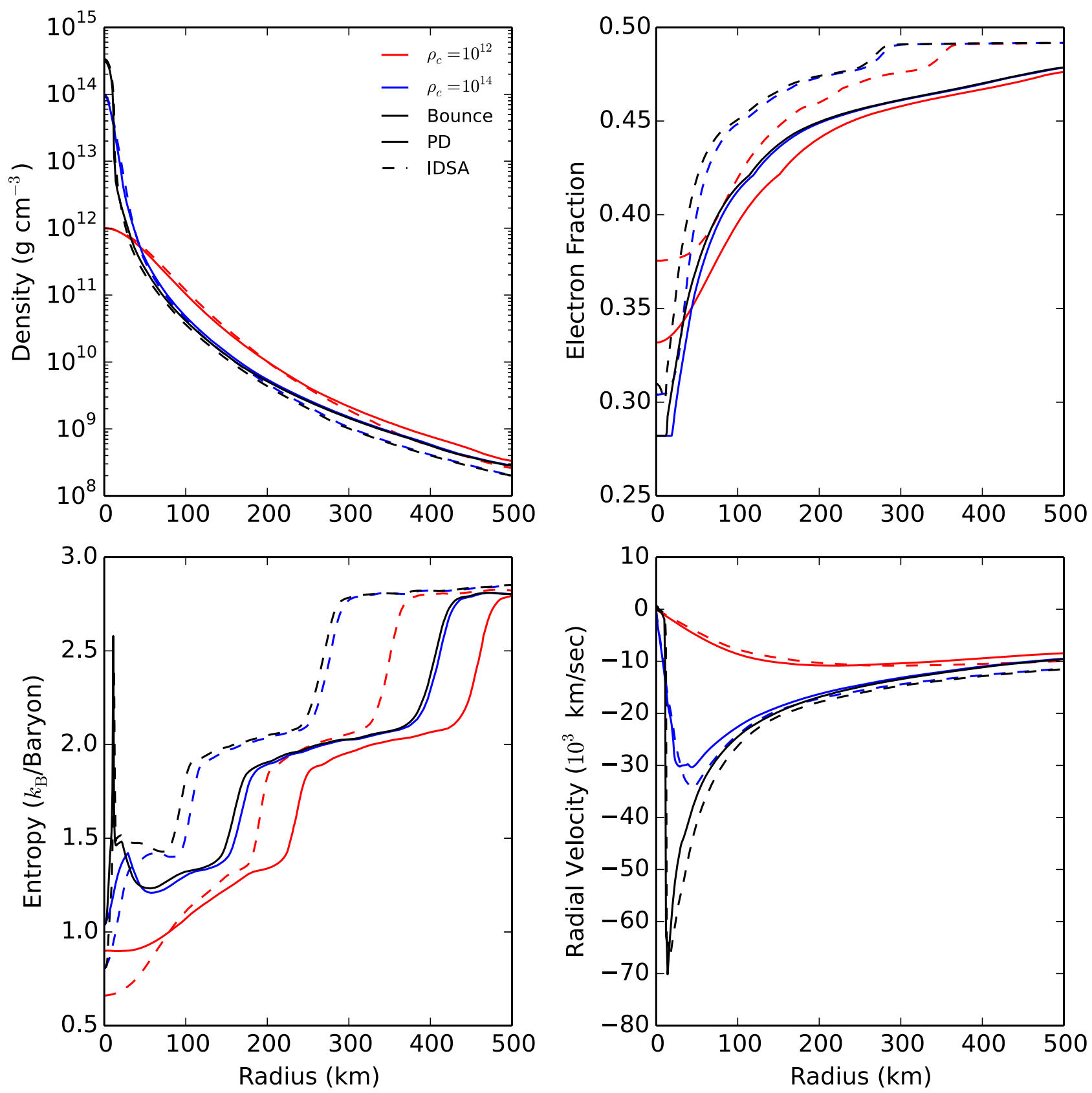

Figure 11. Similar to Figure 10, but for progenitor s15.0.

The kernel of our IDSA solver in FLASH is the same as the solver in AGILE-IDSA. The main differences are from the hydrodynamics, the explicit solver for the diffusion scalar $\alpha$ in Equation (10), the detailed treatment of the EOS, and potentially also the gravity solver. The low-density extension for the EOS in FLASH and differences in the internal energy shift may also provide slightly differences. In principle, we should expect identical results in both codes in 1D. As presented above, although some little differences have been observed, most features are consistent and in nice agreement. Liebendörfer et al. (2005) have performed a code comparison of the Boltzmann solver AGILE-BOLTZTRAN with the VERTEX code that has a variable Eddington factor. Both codes have sophisticated physics input in spherical symmetry but different implementations. As pointed out in Liebendörfer et al. (2005), the different grids in the Lagrangian or Eulerian coordinates produce late-time differences in the shock evolution when the shock runs through shell interfaces. Since FLASH is also an Eulerian code, similar differences between FLASH and AGILE-IDSA as we have found here can be expected.

\subsection{Code Comparisons for the $15 M_{\odot}$ and $20 M_{\odot}$ Progenitors}

The s15 and s20 progenitors from Woosley \& Heger (2007) have been widely studied in the literature, e.g., by Bruenn et al. (2013), Dolence et al. (2015), Suwa et al. (2014), Hanke (2014), Summa et al. (2015), and Melson et al. (2015a). In addition, Lentz et al. (2012b, 2012a) have shown that different neutrino opacities and approximations could lead to different postbounce evolutions by using spherically symmetric AGILE-BOLTZTRAN simulations with the s15 progenitor. Therefore these two progenitor models are very suitable for 

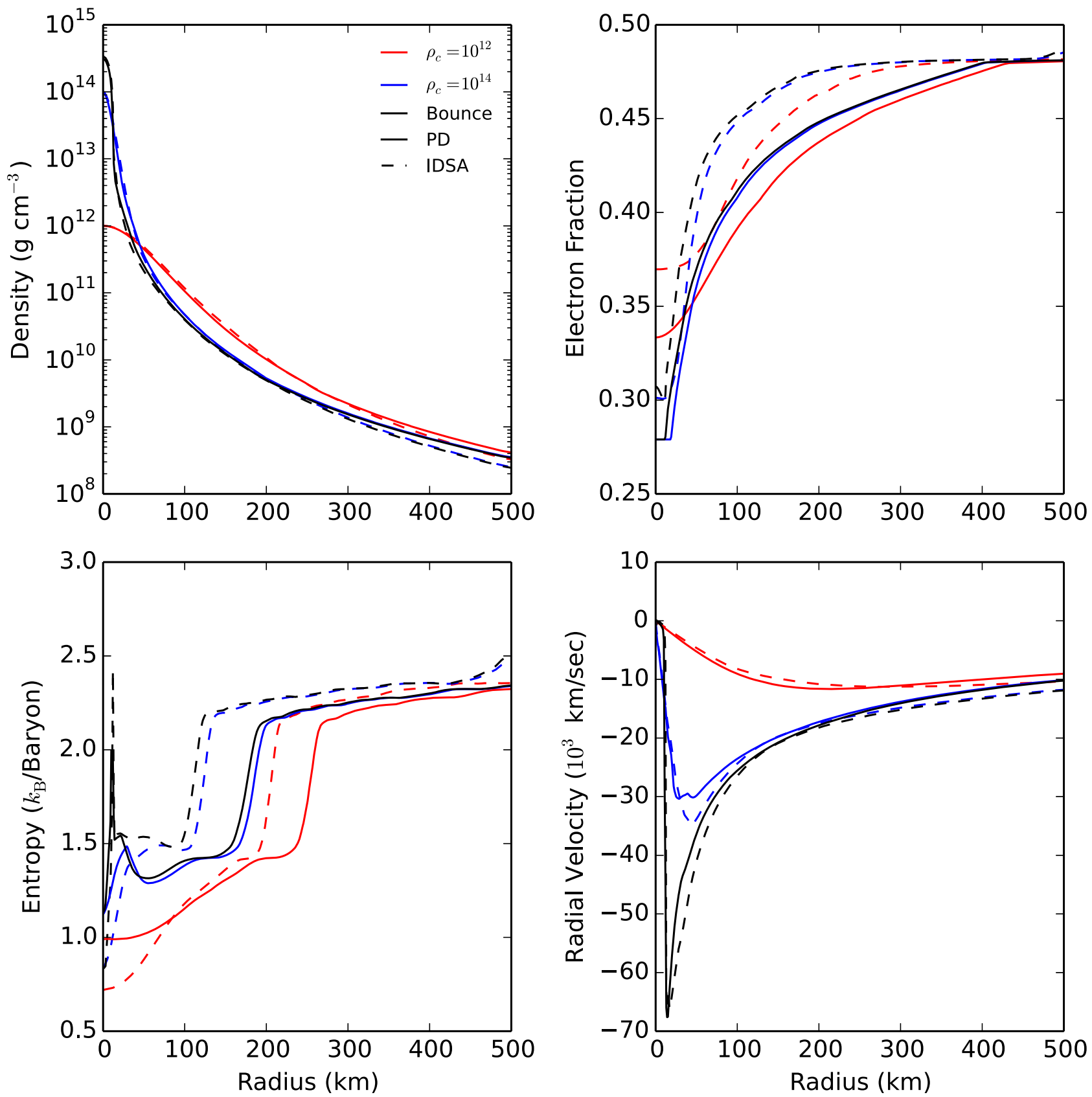

Figure 12. Similar to Figure 10, but for progenitor s21.0.

comparisons among different $\mathrm{SN}$ codes. It is generally agreed that the Vertex-Prometheus (Rampp \& Janka 2002) and CHIMERA (Bruenn et al. 2013) codes use state-of-the-art physics for neutrino transport. Therefore, it is worth performing simulations with these progenitor models to have a direct comparison. However, there is still different physics employed in the above-mentioned works. For instance, CASTRO simulations (Dolence et al. 2015), ZEUS simulations (Suwa et al. 2014), and our FLASH-IDSA simulations are Newtonian, but AGILE-BOLTZTRAN simulations (Lentz et al. 2012b, 2012a), CHIMERA simulations (Bruenn et al. 2013), and Vertex-Prometheus simulations (Hanke 2014; Melson et al. 2015a) are GR or post-Newtonian. Furthermore, Dolence et al. (2015) use Shen's EOS while other groups use the LS220 EOS. Therefore in this section we only give a qualitative discussion.

To evaluate whether the PD scheme could effectively represent NES, we perform three Newtonian AGILE-BOLTZTRAN simulations with the s15 progenitor and the LS220 EOS. The first simulation (model AB-NR) includes the NES, the second simulation (model AB-NR-NoNES) ignores the NES, and the third simulation (model AB-NR-Mix) includes NES only in the collapse phase. Figure 8 shows the evolutions of the shock radius and neutrino luminosity of these three simulations together with FLASH-IDSA simulations with and without PD. Ignoring NES (models FLASH-IDSA and AB-NoNES in Figure 8) gives a short period of shock expansion at $\sim 10 \mathrm{~ms}$ postbounce. A signature from this can also be seen in the 

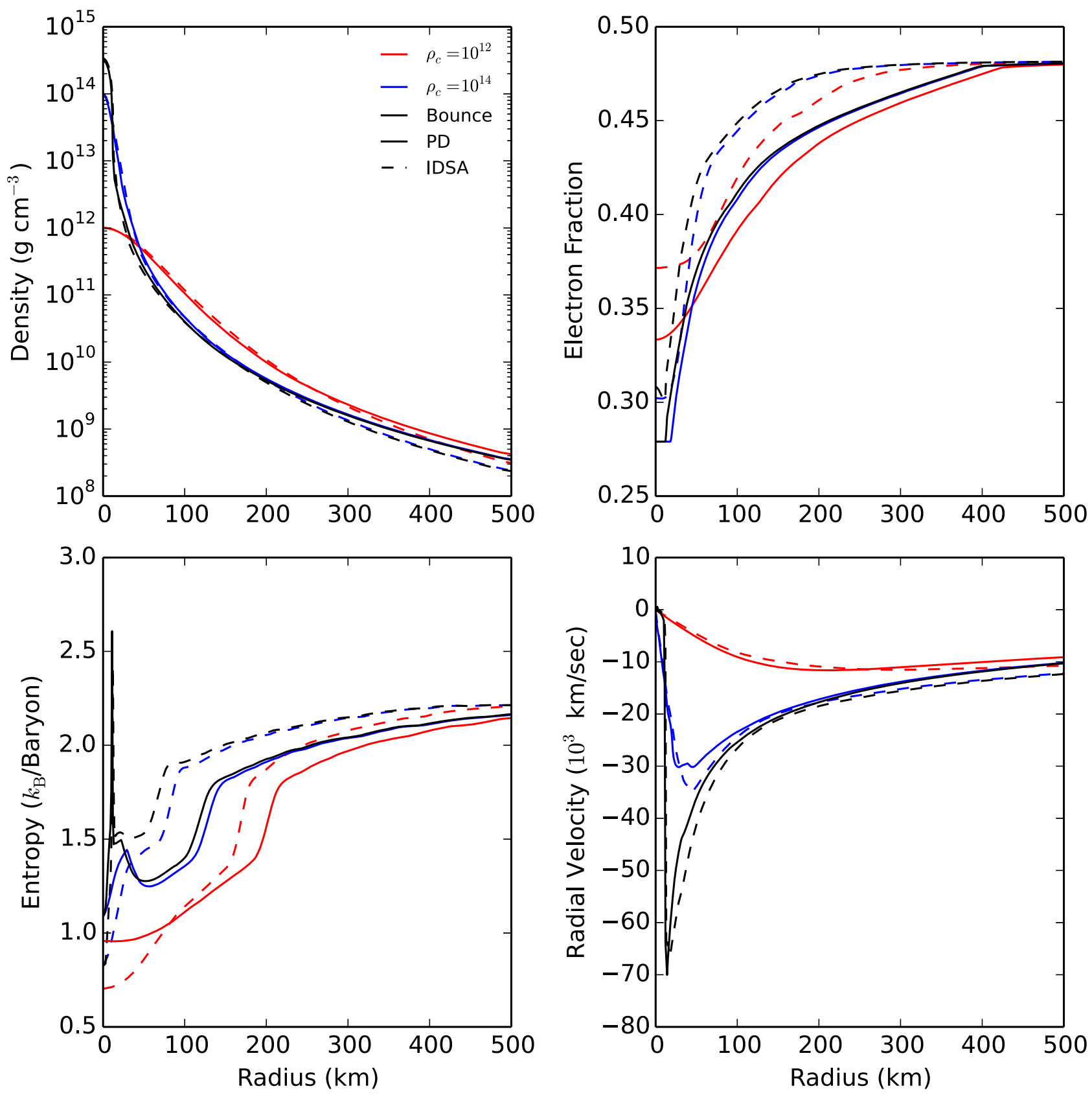

Figure 13. Similar to Figure 10, but for progenitor s27.0.

electron antineutrino luminosity. This behavior is consistent with the model "N-ReduceOp" in Lentz et al. (2012b), and the evolutions of the shock and electron-type neutrino luminosity of our FLASH-IDSA simulation without PD are also consistent with our AGILE-BOLTZTRAN simulation (AB-NR-NoNES).

The model AB-NR-Mix is nearly identical to the model AB$\mathrm{NR}$, demonstrating that NES is important mainly during the collapse phase (Figure 8). Fischer et al. (2012) showed that NES plays a role in the late-time PNS cooling and deleptonization $(>1 \mathrm{~s})$ after the explosion. However, our simulations focus only on the first few hundred milliseconds. The PD scheme in model FLASH-PD greatly improves the postbounce simulation, making our FLASH-IDSA simulations closer to the AGILE-BOLTZTRAN simulation with full neutrino reactions. Although there is no perfect match, we conclude that the use of the PD scheme in the collapse phase could effectively take into account the NES. It should be noted that heavy neutrinos are treated by a simple leakage scheme, which could lead to some difference in our IDSA simulations as well.

In Figure 9, we show the evolution of the shock radius and neutrino signatures of our 2D FLASH-IDSA simulations of the s15 and s20 progenitors from Woosley \& Heger (2007). To get a fair comparison, we enable the PD scheme during collapse and use the LS220 EOS. Both 2D models explode at $\sim 300 \mathrm{~ms}$ postbounce (see Table 4 ). The explosion time, $t_{400}$, is defined by the time when the averaged shock radius exceeds $400 \mathrm{~km}$ and never recedes at the end of the simulation. Overall, the evolutions of shock radius are similar to the models B15WH07 and B20-WH07 in Bruenn et al. (2013) except that the CHIMERA simulations show earlier explosions. It should be noted that we use the old neutrino interactions from Bruenn 

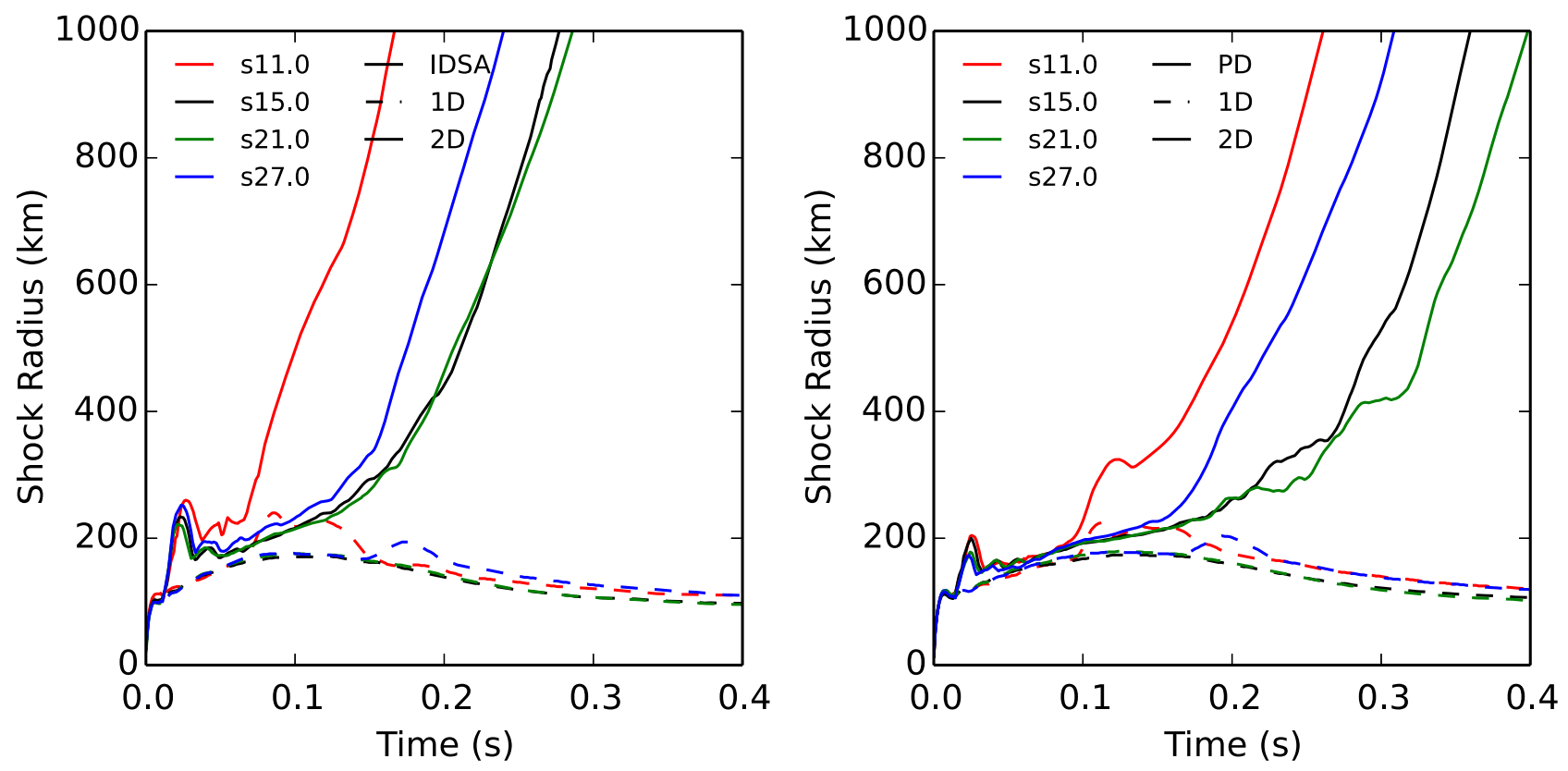

Figure 14. Average shock radius vs. postbounce time for different progenitors and neutrino transport approximations. All models use the DD2 EOS. Different colors indicate different progenitor models. The left panel represents simulations without the PD and the right panel represents simulation with an effective inclusion of NES by the PD. The solid lines show 2D simulations and the dashed lines show 1D simulations.

(1985) and that our simulations are Newtonian. Müller et al. (2012b) have shown that GR effects could enlarge the neutrino luminosities and therefore make it easier to explode. The model s20-2007 in Hanke (2014) and Melson et al. (2015a) shows a similar explosion time to our model s20, but the shock radius at $\sim 150 \mathrm{~ms}$ shrinks to $\sim 150 \mathrm{~km}$ due to GR effects. The reasons for the differences between Vertex-Prometheus and CHIMERA are still unclear, but the overall features for the progenitor s20 are still rather similar. However, the model s15-2007 in Hanke (2014) shows a very different result. The shock stalls for $\sim 500 \mathrm{~ms}$ and then explodes at around $\sim 600 \mathrm{~ms}$.

On the other hand, 2D CASTRO and ZEUS Newtonian simulations by Dolence et al. (2015) and Suwa et al. (2014) did not obtain an explosion with the s15 and s20 progenitors. Our 2D simulations show a fast shock expansion after the prompt convection ( $20 \mathrm{~ms}$, see Figure 9). This is similar to what was observed in Dolence et al. (2015) but somewhat less dramatic. The prompt convection and fast shock expansion coincide with an oscillation of the electron antineutrino luminosity at 10-20 ms (see Figure 9 and Figure 6 of Dolence et al. 2015). These could be caused by the reduced opacity or incomplete neutrino interactions as discussed before. Note that Dolence et al. (2015) use the Shen EOS, which is considered more difficult to lead to explosions than LS220 (Couch 2013; Suwa et al. 2013).

Suwa et al. (2014) also use the IDSA (without PD) but with spherical coordinates and the "RbR" approach. In principle, we should expect similar results, but the nonexplosion of s15 and s20 in Suwa et al. (2014) suggests that the different hydrodynamics code, geometry, resolutions, and multidimensional neutrino transport approximation may also cause significant differences. Suwa et al. (2014) use 300 logarithmically spaced radial zones (from 1 to $5000 \mathrm{~km}$ ) and 1.4 angular resolution. This is roughly three times lower than our simulations. A detailed code comparison is therefore necessary.

\section{MULTI-PROGENITOR STUDY}

We perform 1D and 2D simulations with s11.0, s15.0, s21.0, and s27.0 progenitor models from Woosley et al. (2002). Simulations run from the prebounce core collapse to several hundred milliseconds postbounce with and without the PD in the collapse phase. The former is important in order to effectively take NES into account. Table 3 shows the core properties of these four progenitors during collapse based on 1D simulations. A summary of all performed simulations is shown in Table 4. The model abbreviations in Tables 3 and 4 are defined by a set of letters and numbers: the first two characters define the dimension of the model (1D or 2D); the first letter after the hyphen denotes the EOS of the model (L for LS220 and D for DD2); the second letter shows the transport scheme during the collapse (A for IDSA and P for PD); and the last two numbers specify the mass of the investigated progenitor model. A " -07 " at the end shows progenitor models from Woosley \& Heger (2007), otherwise they are from Woosley et al. (2002). For instance, model 1D-DA15 means a 1D simulation of the s15.0 progenitor with DD2 EOS and using the IDSA in the collapse phase (i.e., effectively without NES). When we refer to models DA, we consider all models with "DA" in their abbreviations.

\subsection{Stellar Collapse and Core Bounce}

Simulations are started from the non-rotating, solarmetallicity pre-supernova progenitors from Woosley et al. (2002) without artificial perturbation. Couch \& Ott (2013) and Mueller \& Janka (2015) show that small perturbations on the $\mathrm{Si} / \mathrm{O}$ interface during collapse could amplify post-shock turbulence and turn a model that failed to explode toward a successful explosion. In addition, Couch et al. (2015) 

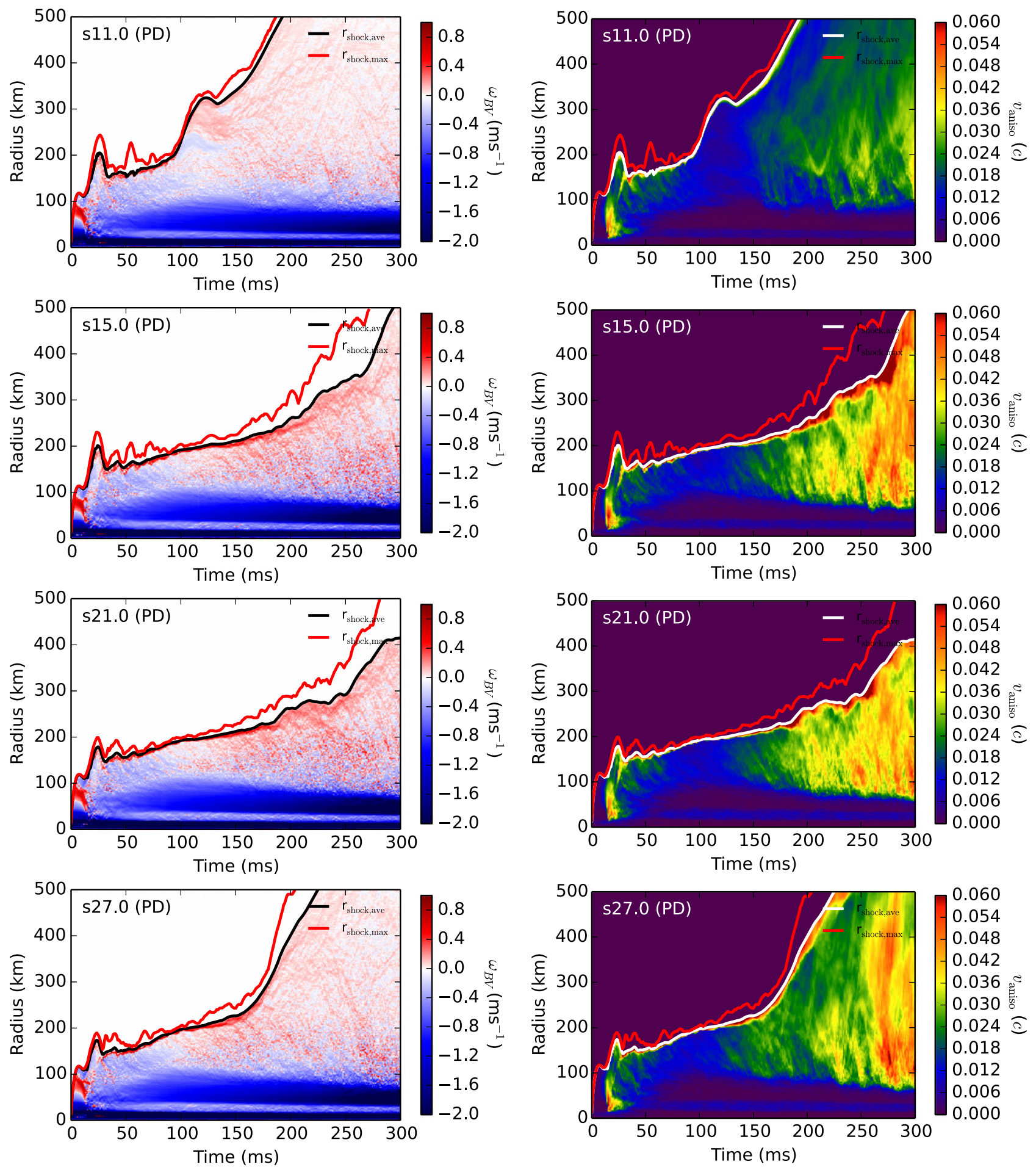

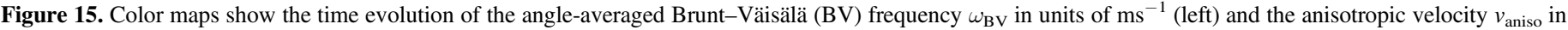

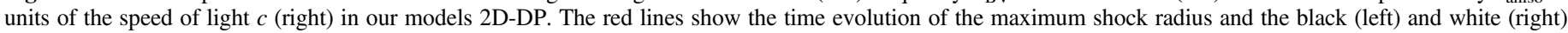
lines show the time evolution of the averaged shock radius.

performed 3D simulations of the final few minutes of iron core growth in a massive star that includes Si shell burning. The results suggest that the non-spherical progenitor structure may have a strong impact on neutrino-driven explosions. In our models, we do not include these non-spherical features, and therefore the 2D simulations during collapse show nice agreement with 1D simulations in all models. However, we will show that spherical variations due to different levels of electron deleptonization or neutrino reactions during collapse may also have a significant impact on neutrinodriven explosions.

Figures 10-13 show the radial density, electron fraction, entropy, and radial velocity evolutions of 2D models with an HS(DD2) EOS based on the IDSA or the PD at different times during the collapse phase. The 1D data are not shown because they are barely distinguishable from the 2D data before bounce. 

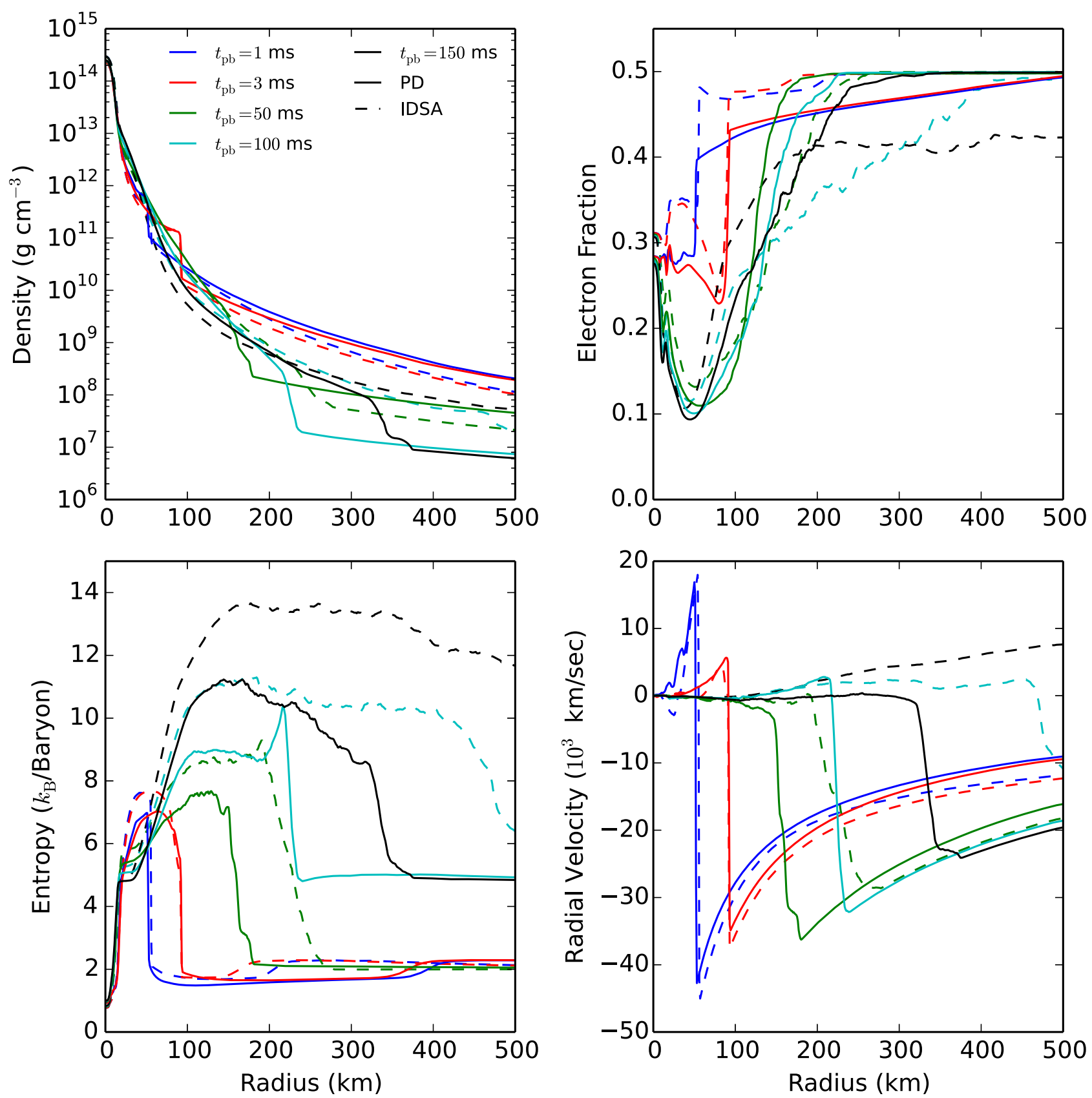

Figure 16. Angle-averaged radial profiles of progenitor s11.0 at different postbounce times. All models are evolved in 2D and use the DD2 EOS. Different colors indicate different postbounce times. The solid lines show simulations with the PD and the dashed lines show simulations without the PD.

In the bounce profiles, the models DP show a slightly lower central density but a higher density outside the iron core, due to an earlier bounce time.

The core evolutions of different progenitors behave qualitatively similarly during collapse, but show quantitive differences for the central electron fraction, central density, and bounce shock locations. Table 3 summarizes important quantities for the four different progenitors during the collapse phase. Due to a higher initial central density of the progenitor $\mathrm{s} 11.0$, the central density of $\mathrm{s} 11.0$ reaches $\rho_{c}=10^{11} \mathrm{~g} \mathrm{~cm}^{-3}$ $\sim 100 \mathrm{~ms}$ earlier than other progenitors. Once the core densities in different progenitors reach the same $\rho_{c}$, the more massive progenitors collapse faster than other progenitors.

In addition, since the electrons are highly degenerate and can only gain energy, this means that neutrinos lose their energy through NES and therefore escape more easily, accelerating the collapse process (Bethe 1990). Therefore models DP (with effective NES) collapse faster than models DA (without NES) and have a lower $Y_{e}$ (more $e$-captures), $Y_{l}$ ( $+\nu$-escape), and $\rho_{c}$ at core bounce.

Core bounce is defined here by the first time when the maximum density in the core exceeds $2 \times 10^{14} \mathrm{~g} \mathrm{~cm}^{-3}$ and the maximum peak entropy is above $3 k_{\mathrm{B}}$ baryon $^{-1}$. At bounce, the bounce shock emerges at $\sim 0.55 M_{\odot}$ (defined by the mass enclosed within the shock front at bounce) in models DP and at $\sim 0.75 M_{\odot}$ in models DA. Since the core mass is proportional to $Y_{e}^{2}$ (Yahil \& Lattimer 1982), models DA have higher core mass than models DP at bounce. The highest infall velocity at bounce is about $\sim 70,000 \mathrm{~km} \mathrm{~s}^{-1}$ in all progenitor models. It should be noted that the $\mathrm{Si} / \mathrm{O}$ interface, which corresponds to 

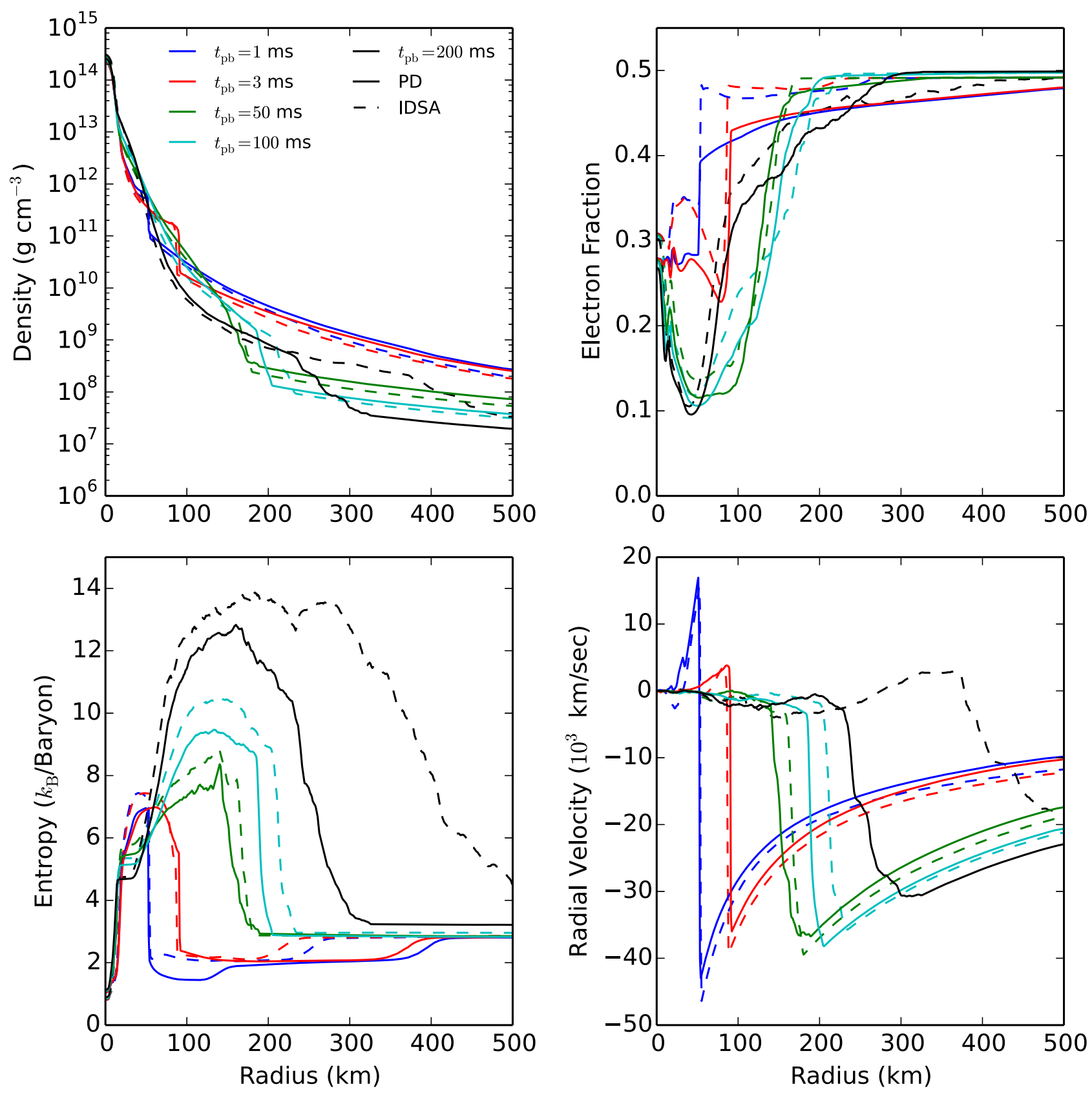

Figure 17. Similar to Figure 16, but for progenitor s15.0.

the region of high entropy gradient at about $100-400 \mathrm{~km}$ in Figures $10-13$, is at different radii for models DA and DP due to different bounce times, since the bounce time directly sets the location of the $\mathrm{Si} / \mathrm{O}$ interface. These differences will strongly influence the shock evolution after bounce.

\subsection{Shock Expansion and Instabilities}

Figure 14 shows the angle-averaged shock radius as a function of time in our 1D and 2D simulations with the DD2 EOS. The shock radius of a sample ray is defined here by the largest radius where the entropy is above $s_{\min }=$ $4.5 k_{\mathrm{B}}$ baryon $^{-1}\left(s_{\min }=6 k_{\mathrm{B}}\right.$ baryon $^{-1}$ for progenitor models s11.0 and s27.0) after the postbounce time was reached, $t_{\mathrm{pb}}=50 \mathrm{~ms}$. In $2 \mathrm{D}$ simulations, we sample 180 radial rays.
Before $50 \mathrm{~ms}$ postbounce, the shock front is defined by the minimum radial infall velocity.

1D and 2D models behave very similarly in the first few milliseconds until the bounce shock passes through the neutrino sphere at $\sim 10 \mathrm{~ms}$. At that time, a prompt entropy- and electrondriven convection occurs and makes the $2 \mathrm{D}$ simulations different from 1D. Later on, this prompt convection causes a fast shock expansion and changes the shock radius from $r_{\mathrm{sh}} \sim 100 \mathrm{~km}$ to $r_{\mathrm{sh}} \sim 200 \mathrm{~km}$ at $t_{\mathrm{pb}} \sim 20 \mathrm{~ms}$ (see Figure 14). While the prompt convection is caused by the negative entropy and $Y_{e}$ gradient, it should be noted that this early fast shock expansion during $t_{\mathrm{pb}} \sim 10-20 \mathrm{~ms}$ may be amplified by our incomplete neutrino interactions and the old neutrino opacities, and by the grid-effect in cylindrical coordinates in consideration of the multidimensional treatment of neutrino diffusion, since a Rayleigh-Taylor 

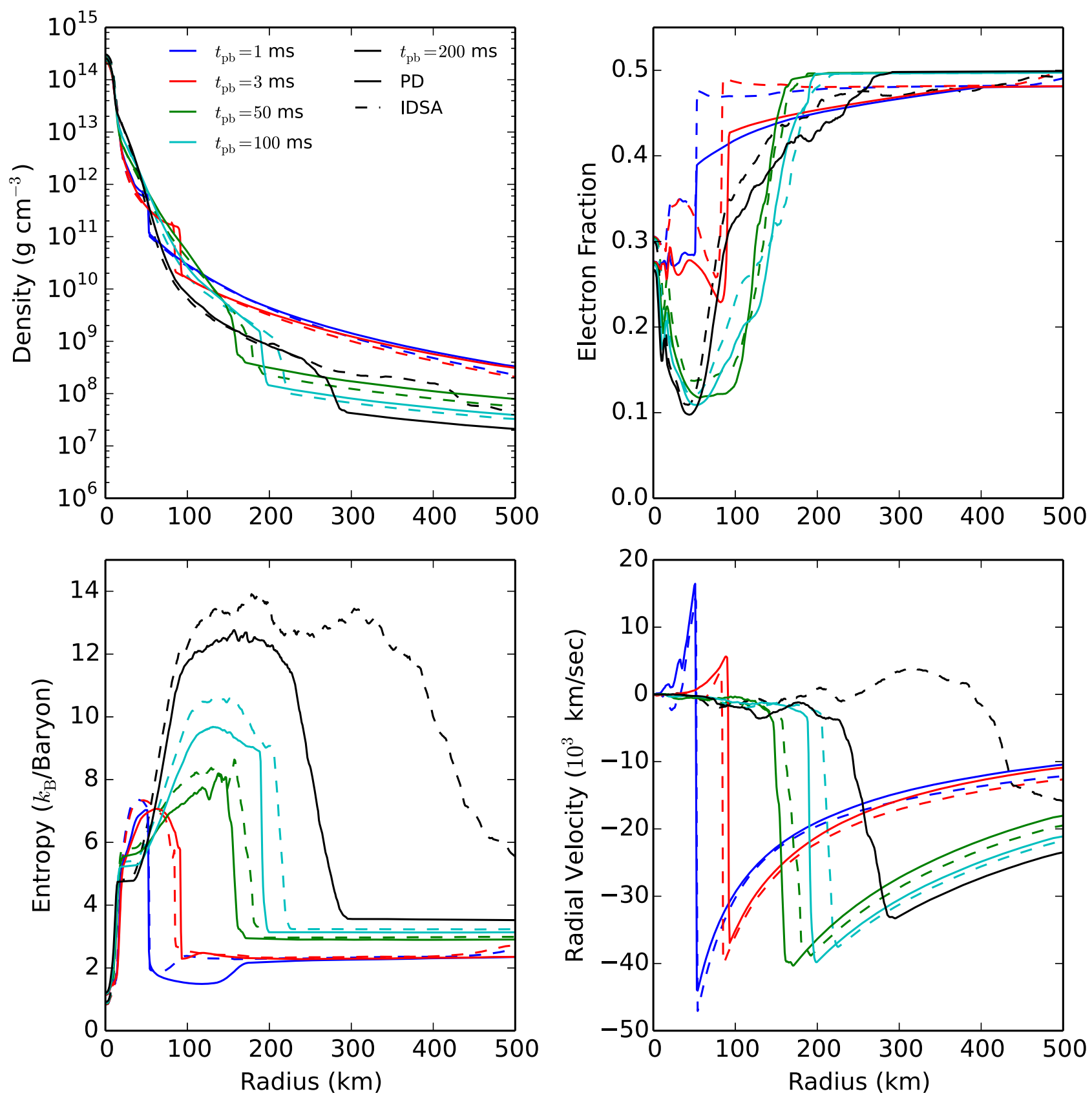

Figure 18. Similar to Figure 16, but for progenitor s21.0.

bubble along the diagonal axis is observed at that time. A similar fast shock expansion is observed in Dolence et al. (2015) as well, but is not obvious in any other 2D simulation with spherical coordinates. However, unlike the results in Dolence et al. (2015), the shock radius in our simulations shrinks back to $r_{\mathrm{sh}} \sim 150 \mathrm{~km}$ after this prompt convection at $t_{\mathrm{pb}} \sim 50 \mathrm{~ms}$.

The prompt and late convection can be understood from the local stability analysis via the Ledoux criterion (Ledoux 1947),

$$
C_{L}=-\left(\frac{\partial \rho}{\partial P}\right)_{s, Y_{l}}\left[\left(\frac{\partial P}{\partial s}\right)_{\rho, Y_{l}}\left(\frac{d s}{d r}\right)+\left(\frac{\partial P}{\partial Y_{l}}\right)_{\rho, s}\left(\frac{d Y_{l}}{d r}\right)\right],
$$

where we assume $Y_{l} \sim Y_{e}$ for simplicity. The Brunt-Väisälä (BV) frequency, $\omega_{\mathrm{BV}}$, describes the linear growth frequency for convection. Following the definition of Buras et al. (2006) and
Ott et al. (2013), one obtains

$$
\omega_{\mathrm{BV}}=\operatorname{sign}\left(C_{L}\right) \sqrt{\left|\frac{C_{L}}{\rho} \frac{d \Phi}{d r}\right|},
$$

where $\Phi$ is the local gravitational potential and the approximation $d \Phi / d r \sim-G M(r) / r^{-2}$ was used. Once convection is active, another useful quantity to describe its strength is the anisotropic velocity. We follow the definition from Takiwaki et al. (2012) and define the anisotropic velocity as

$$
v_{\text {aniso }}=\sqrt{\frac{\left\langle\rho\left[\left(v_{r}-\left\langle v_{r}\right\rangle_{4 \pi}\right)^{2}+v_{\phi}^{2}\right]\right\rangle_{4 \pi}}{\langle\rho\rangle_{4 \pi}}} .
$$



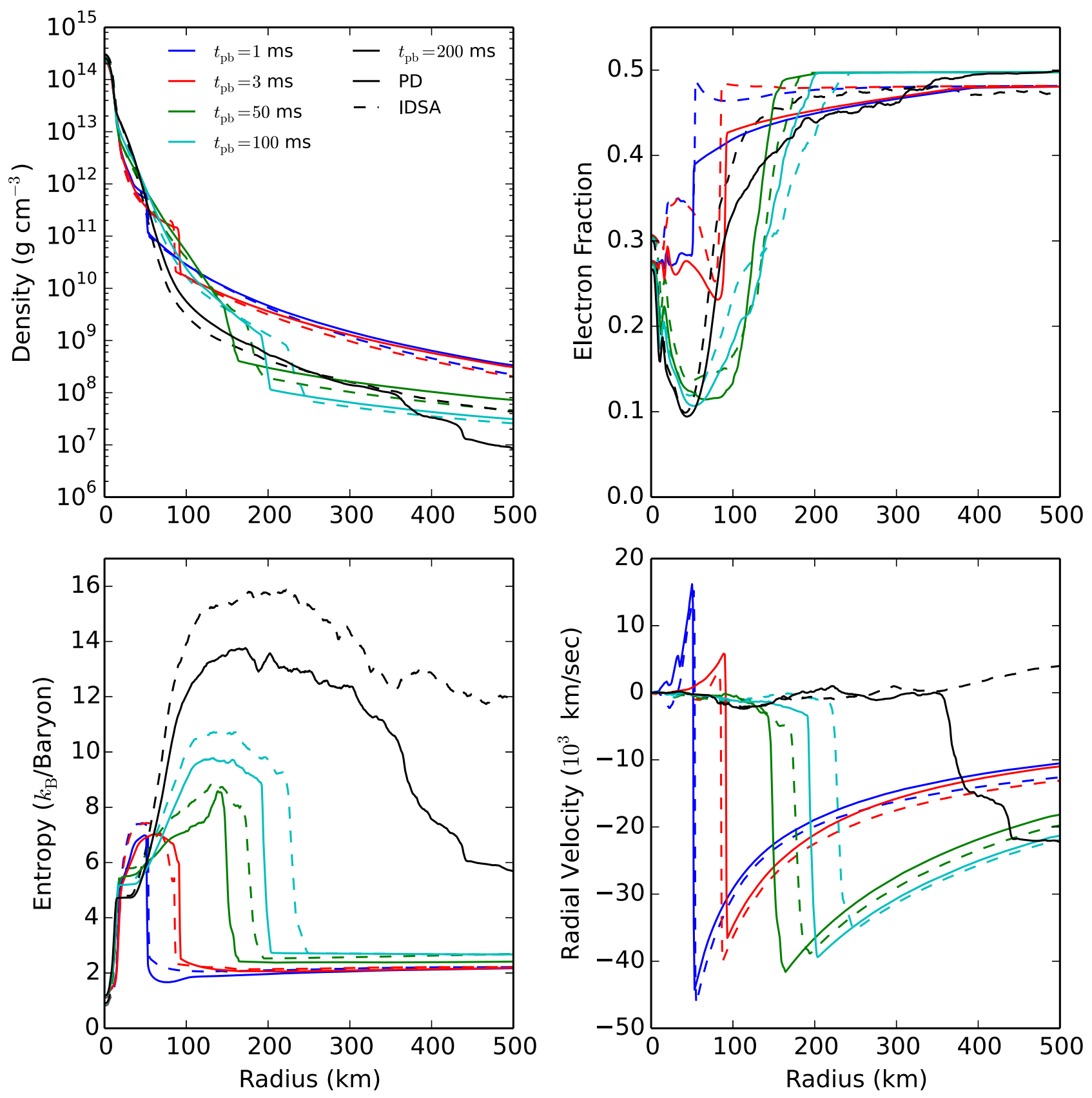

Figure 19. Similar to Figure 16, but for progenitor s27.0.

In Figure 15, we show the evolution of the angle-averaged BV frequency and anisotropic velocity of our models DP. The $\mathrm{BV}$ frequencies are positive and high when the shock breaks through the neutrino sphere and the prompt convection happens at $\sim 20 \mathrm{~ms}$. Starting from the core regions, the anisotropic velocities become very strong after the prompt convection has developed, and therefore drive the fast shock expansion at $\sim 20 \mathrm{~ms}$. However, the convection stops and the anisotropic velocity returns to small values at $\sim 50 \mathrm{~ms}$. Models DA behave similarly but on a different timescale due to different shock evolutions. We note that our estimate of $C_{L}$ may be incorrect at small radii, where neutrinos are trapped, due to the neglect of the contribution from neutrinos in $Y_{l}$, but the overall features should be the same.
For a given progenitor model, the bounce shocks have a similar shock strength in both models DA and DP. The difference in shock radius in models DA and DP during the prompt convection is mainly due to the difference in the bounce time, since a different bounce time leads to a different shock strength when the bounce shock reaches the location of the negative entropy gradient. Since convection is suppressed in 1D, we do not observe this prompt convection in 1D models. However, the prompt convection in 2D models leads to a larger shock radius than in 1D models at $t_{\mathrm{pb}} \sim 50 \mathrm{~ms}$.

After $t_{\mathrm{pb}} \sim 50 \mathrm{~ms}$, the shock stalls at $r_{\mathrm{sh}} \sim 150-200 \mathrm{~km}$ for another $\sim 50-100 \mathrm{~ms}$ (except for model 2D-DA11). It is no surprise that all 1D models fail to explode due to the lack of convection. However, at first it seems surprising that all our 2D 


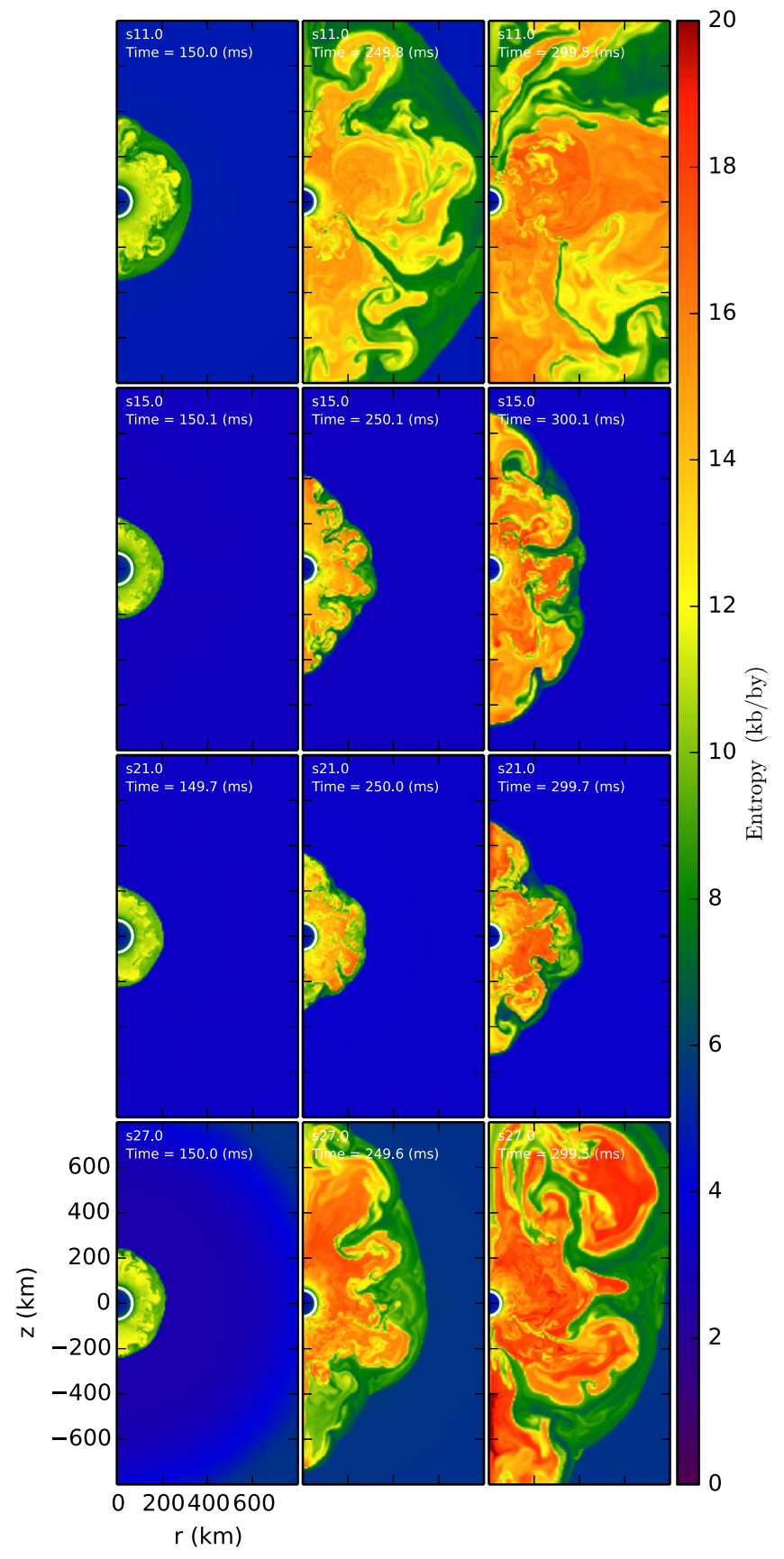

Figure 20. Entropy distributions of our 2D models with the PD and the DD2 EOS (see Table 4). Each frame shows a section of the domain spanning $800 \mathrm{~km}$. The color scale indicates the entropy in $k_{\mathrm{B}} /$ baryon.

models explode within $\sim 100-300$ ms postbounce regardless of the progenitor mass and the inclusion of NES during collapse. But on second thoughts, one has to consider that our simulations are Newtonian (comparing with Bruenn et al. 2001; Liebendörfer et al. 2001b; Müller et al. 2012b) and do not include the most recent electron capture rates (Hix et al. 2003; Langanke et al. 2003). In general, Newtonian simulations disfavor explosions compared to general-relativistic simulations (Müller et al. 2012b), and also the old neutrino interactions and opacities could make our simulations too optimistic with respect to explosions. Furthermore, we are using the DD2 EOS, which has more realistic nuclear matter properties than the LS220 EOS that was used in the abovementioned simulations. In the Appendix we show that DD2 leads in our simulations to more favorable conditions for explosions than LS220. Table 4 summarizes the essential parameters of our $1 \mathrm{D}$ and $2 \mathrm{D}$ simulations at the end of the simulations. Model 2D-DA11 is the fastest explosion model, exploding already at $t_{400}=86 \mathrm{~ms}$ by neutrino-driven convection. For the other DA models, the explosion time is $t_{400} \sim 160-190 \mathrm{~ms}$. In addition, because of the effective NES, models DP evolve more slowly and explode at a late $t_{400} \sim 200-280 \mathrm{~ms}\left(t_{400}=170 \mathrm{~ms}\right.$ for model 2D-DP11, see Table 4 for detailed information).

In model 2D-DP11, the shock radius first expands to $r_{\mathrm{sh}} \gtrsim 300 \mathrm{~km}$ at $\sim 100 \mathrm{~ms}$ postbounce when it reaches the $\mathrm{Si} / \mathrm{O}$ interface, but temporarily drops back to $r_{\mathrm{sh}} \lesssim 300 \mathrm{~km}$ at $\sim 130 \mathrm{~ms}$ postbounce before it explodes. Progenitors s15.0 and s21.0 explode later than progenitors s11.0 and s27.0 but at a similar time in both models 2D-DA and 2D-DP. In Figures 16-19, we compare the average radial profiles of density, entropy, electron fraction, and radial velocity at 1, 3, 50,100 , and $200 \mathrm{~ms}$ postbounce for models DA and DP. We terminate the simulations at $\sim 700-800 \mathrm{~ms}$ postbounce in $1 \mathrm{D}$ and at $\sim 300-500 \mathrm{~ms}$ postbounce in $2 \mathrm{D}$.

The 2D entropy distribution of models 2D-DP is shown in Figure 20 at 150, 250, and $300 \mathrm{~ms}$ postbounce. At $\sim 150 \mathrm{~ms}$, the convection is getting stronger (see Figure 15) but the distribution of shock radius is still spherically symmetric. Later on, at $\sim 200 \mathrm{~ms}$, the SASI start in models 2D-DP15, 21, and 27 but the models of progenitor s11.0 show mainly convection without SASI. The SASI activities can be seen in Figure 21, where we show the normalized coefficients $a_{l}$ of the decomposition of the shock radius $r_{\mathrm{sh}}(\theta)$ into Legendre polynomials $P_{l}$ (Müller et al. 2012a). $a_{l}$ can be calculated from

$$
a_{l}=\frac{2 l+1}{2} \int_{0}^{\pi} r_{\mathrm{sh}}(\theta) P_{l} d \cos \theta
$$

and $a_{0}$ corresponds to the averaged shock radius. For the progenitor s11.0, there is no obvious evidence of SASI activities in either model 2D-DA11 or 2D-DP11. The amplitudes of the normalized coefficients for $l=1,2$, and 3 modes are small and within the same order of magnitude. For progenitors s15.0, s21.0, and s27.0, the SASI activities can be seen and start to grow at $\sim 200 \mathrm{~ms}$ postbounce. After $\sim 200 \mathrm{~ms}$ postbounce, the dipole $(l=1)$ and quadrupole $(l=2)$ modes grow to $a_{l} / a_{0} \sim 0.2$ in the progenitors s15.0 and s21.0, and to $a_{l} / a_{0} \sim 0.1$ in progenitor s27.0. The amplitudes do not show significant differences between models DA and DP, but the starting time of the high growth rates of the amplitudes corresponds to the time of fast shock expansion. SASI activities could also be seen in Figure 22 for the entropy distribution where the $y$ axis is a line starting from the north pole and going to the south pole.

To verify the code convergence, we have also performed a low-resolution run by reducing the angular resolution by a factor of 2 (model 2D-LA15low). We find that the explosion time $t_{400}$ is delayed by $1 \mathrm{~ms}$ and the shock expansion evolves slightly more slowly than for the run with standard resolution (model 2D-LA15). Overall, we find no significant differences between the low-resolution run and the standard run. 

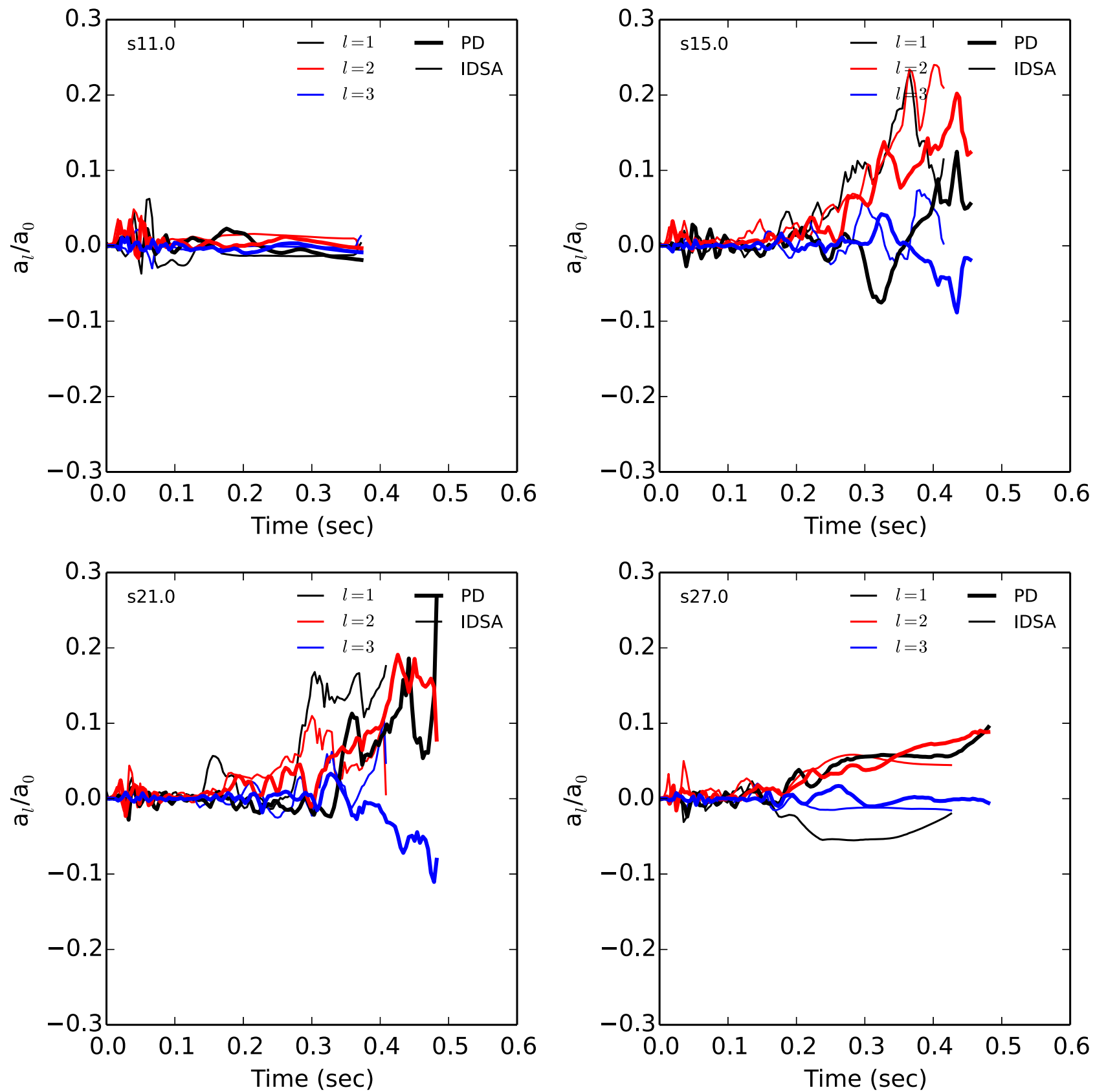

Figure 21. First, second, and third coefficients $a_{1}, a_{2}$, and $a_{3}$ of the spherical decomposition of the shock radius into Legendre polynomials, normalized to the average shock radius $\left(a_{0}\right)$ for different progenitors.

\subsection{Neutrino Heating and Explosion Energy}

The energy released in neutrinos is about $10^{53}$ erg in a typical CCSN (Colgate \& White 1966). To power the observed kinetic energy of a CCSN $\left(\sim 10^{51} \mathrm{erg} \equiv 1 \mathrm{~B}\right)$, the baryonic matter has to absorb $\sim 1 \%$ of the neutrino energy (Bethe \& Pizzochero 1990). However, the explosion energies in most published 2D models are still lower than the standard $1 \mathrm{~B}$ (except for those of Bruenn et al. 2013, 2014). The real explosion energy is not straightforward to calculate and the definition may differ from group to group. Figure 23 shows the "diagnostic energy" of our models 2D-DA and 2D-DP. The diagnostic energy, $E_{\mathrm{dia}}$, is defined by

$$
E_{\mathrm{dia}}=\int_{e_{\mathrm{tot}}>0} \rho e_{\mathrm{tot}} d V
$$

where the volume integration is performed over the region where the total specific energy,

$$
e_{\mathrm{tot}} \equiv\left(e-e_{0}\right)+\frac{1}{2} v^{2}+\Phi,
$$

is positive. $e$ represents the specific internal energy (thermal energy plus binding energy), and $\frac{1}{2} v^{2}$ and $\Phi$ are the specific kinetic and gravitational energies, respectively. $e_{0}$ is the reference energy value that is defined by the minimum specific internal energy at the beginning of the simulation, which leads to a negligible diagnostic explosion energy at the beginning. We have checked that alternative values for the reference energy, which are suggested in the literature, do not have a significant effect on our final results. Depending on progenitor 

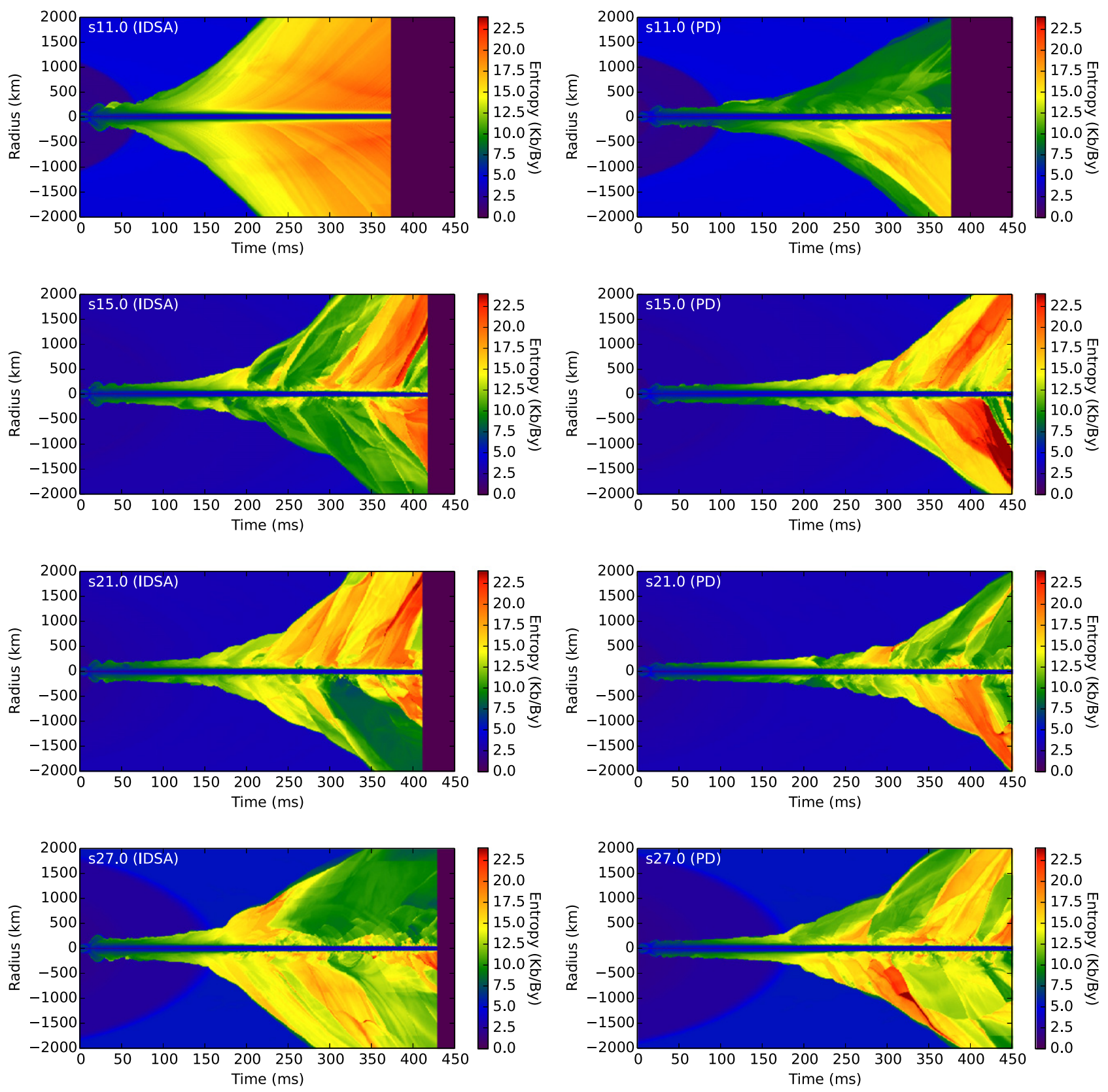

Figure 22. Entropy at the north and the south poles as a function of time for different progenitor masses and for models DA (left) and DP (right) in Table 4.

and simulation domain, $e_{0}$ is about $\sim 10^{17} \mathrm{erg} \mathrm{g}^{-1}$. We find that the diagnostic energy is insensitive to $e_{0}$ if we make it a few times larger or smaller. Note that this expression does not take into account properly the different binding energy contributions from different nuclei to the thermal energy. Therefore the socalled recombination energy is not included. $e$ corresponds only to the thermal energy that actually should be used in $E_{\mathrm{dia}}$, if the composition is dominated by ${ }^{12} \mathrm{C}$ or if it has a similar average binding energy to ${ }^{12} \mathrm{C}$. The diagnostic explosion energies (Figure 23) of our models 2D-DP are between 0.1 B and $0.4 \mathrm{~B}$ at $\sim 400 \mathrm{~ms}$ postbounce and are still increasing at the end of our simulations. The models 2D-DA, which ignored the NES in the collapse phase, have a much stronger explosion energy, $E_{\text {exp }} \sim 0.2-0.8 \mathrm{~B}$ at $\sim 400 \mathrm{~ms}$ postbounce. This result is consistent with the 1D results in Bruenn (1989b). It should be noted that the only difference between models 2D-DA and 2D-DP is the deleptonization method during collapse. The postbounce physics employed is identical in both models 2DDA and 2D-DP, suggesting that the initial conditions at bounce may dramatically affect the postbounce evolutions. Furthermore, note that it is difficult to compare the results of models DP with other groups because there are differences in the input physics and methods. Additionally, we show the diagnostic explosion energies of models 2D-LA15 and 2D-LA15low for a comparison with LS220 EOS and with low resolution. We find that the model with LS220 EOS leads to a slightly lower diagnostic explosion energy due to a delay of the explosion. The low-resolution model also gives a similar result, suggesting 


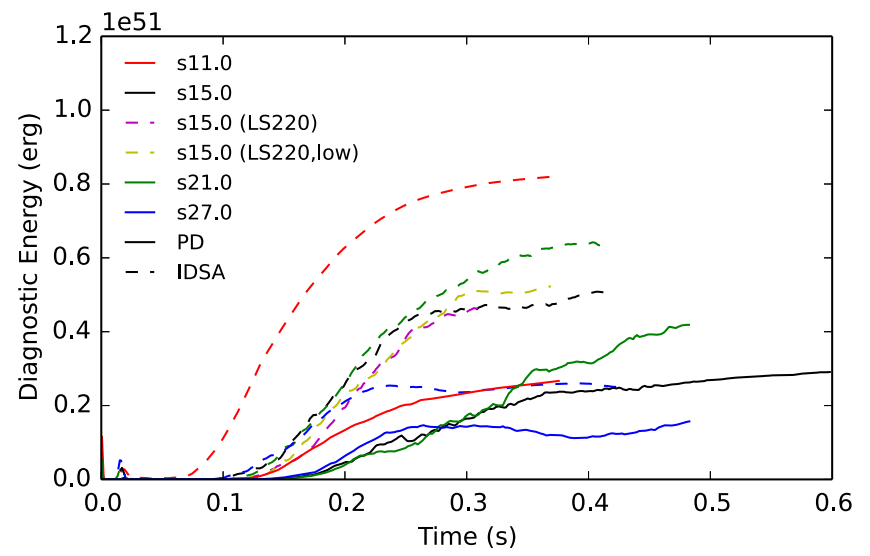

Figure 23. Diagnostic explosion energies defined in Section 4 as a function of time. Different colors represent the different progenitor models. Solid lines represent the models 2D-DP and dashed lines indicate the models 2D-DA in Table 4. The magenta and yellow lines use LS220 EOS, while other lines use DD2 EOS.
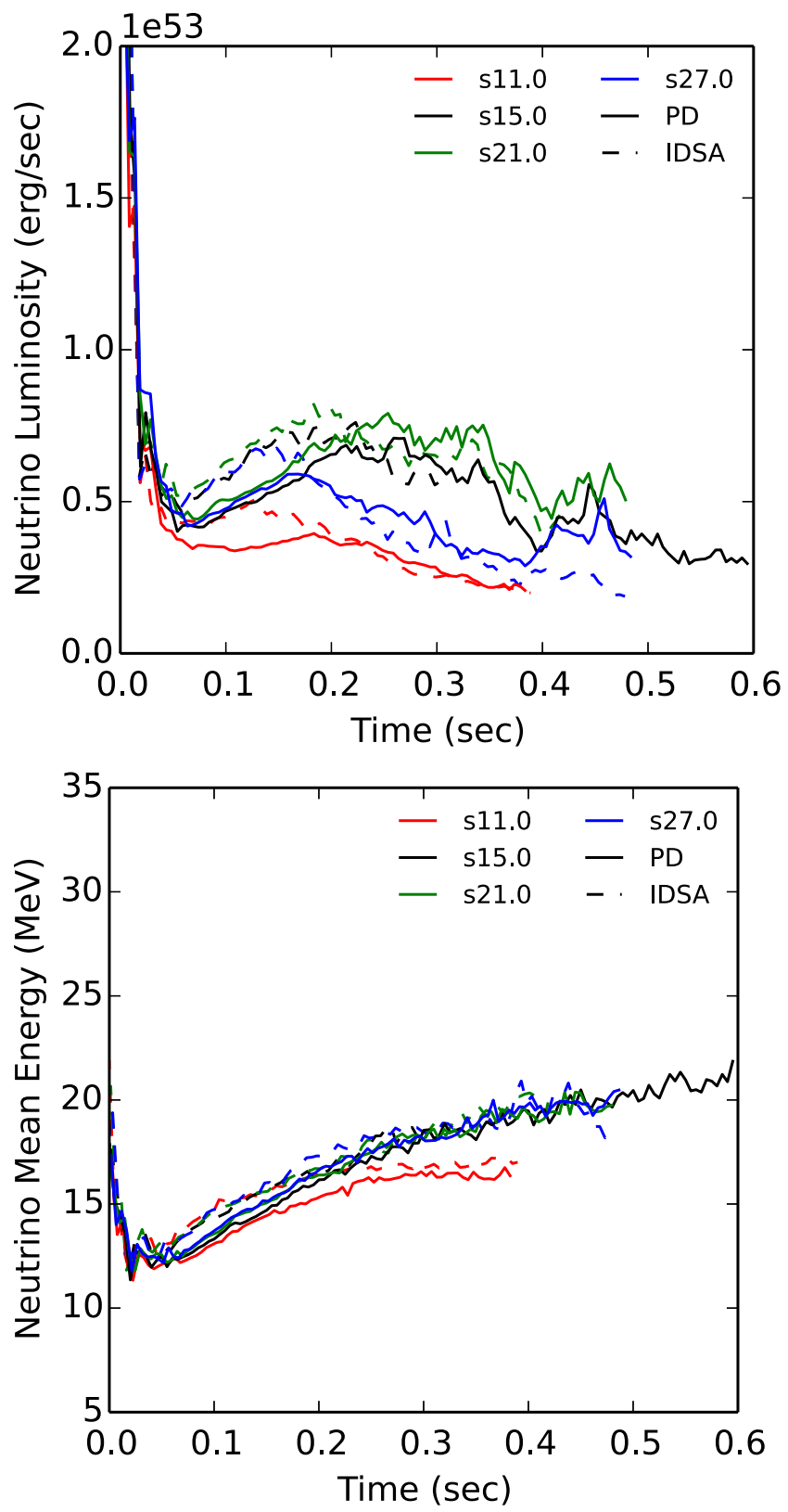

that the fast explosion in our models is not due to insufficient resolution (Figure 23).

Ugliano et al. (2012), Nakamura et al. (2014), and Perego et al. (2015) suggest that there is a correlation of the compactness parameter with the explosion energy. We do see this trend in our simulations except for the progenitor s11.0, if we define the compactness parameter at an enclosed mass of $1.75 M_{\odot}$, i.e., $\xi_{1.75} \equiv \frac{1.75}{R\left(M=1.75 M_{\odot}\right) / 1000 \mathrm{~km}}$ (O'Connor \& Ott 2011). In our simulations, the progenitor s11.0, which has the lowest compactness parameter, has the second highest diagnostic explosion energy (see Tables 3 and 4). However, this trend in our simulations will disappear if we use $\xi_{2.5}$. It should be noted that we only have four progenitor models in our simulations, and the correlations found in Ugliano et al. (2012) and Perego et al. (2015) show a huge scatter between different models, indicating that the compactness parameter oversimplifies the complexity of the explosion mechanism.
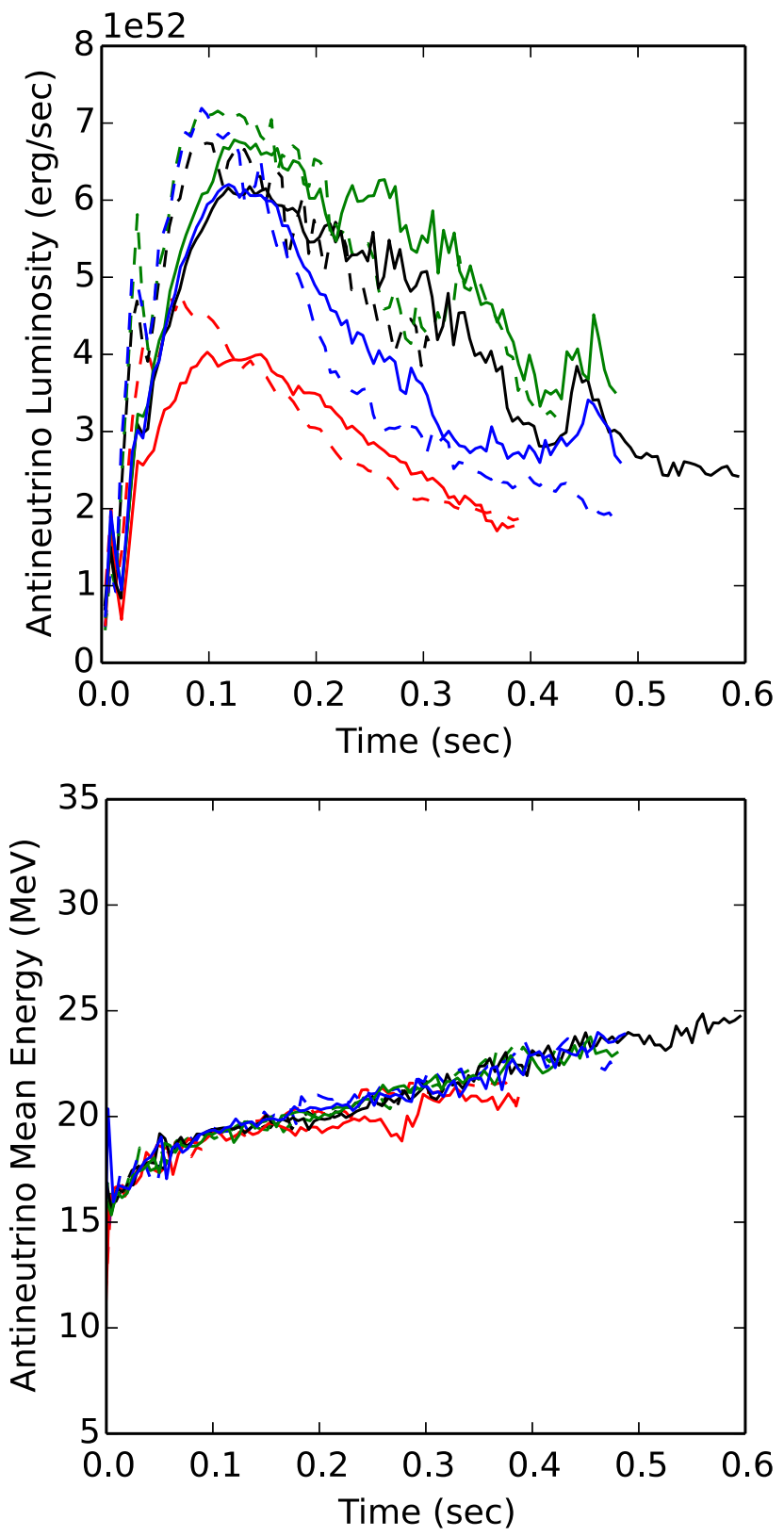

Figure 24. Similar to Figure 7, but for the models DP (solid lines) and the models DA (dashed lines) in Table 4. 


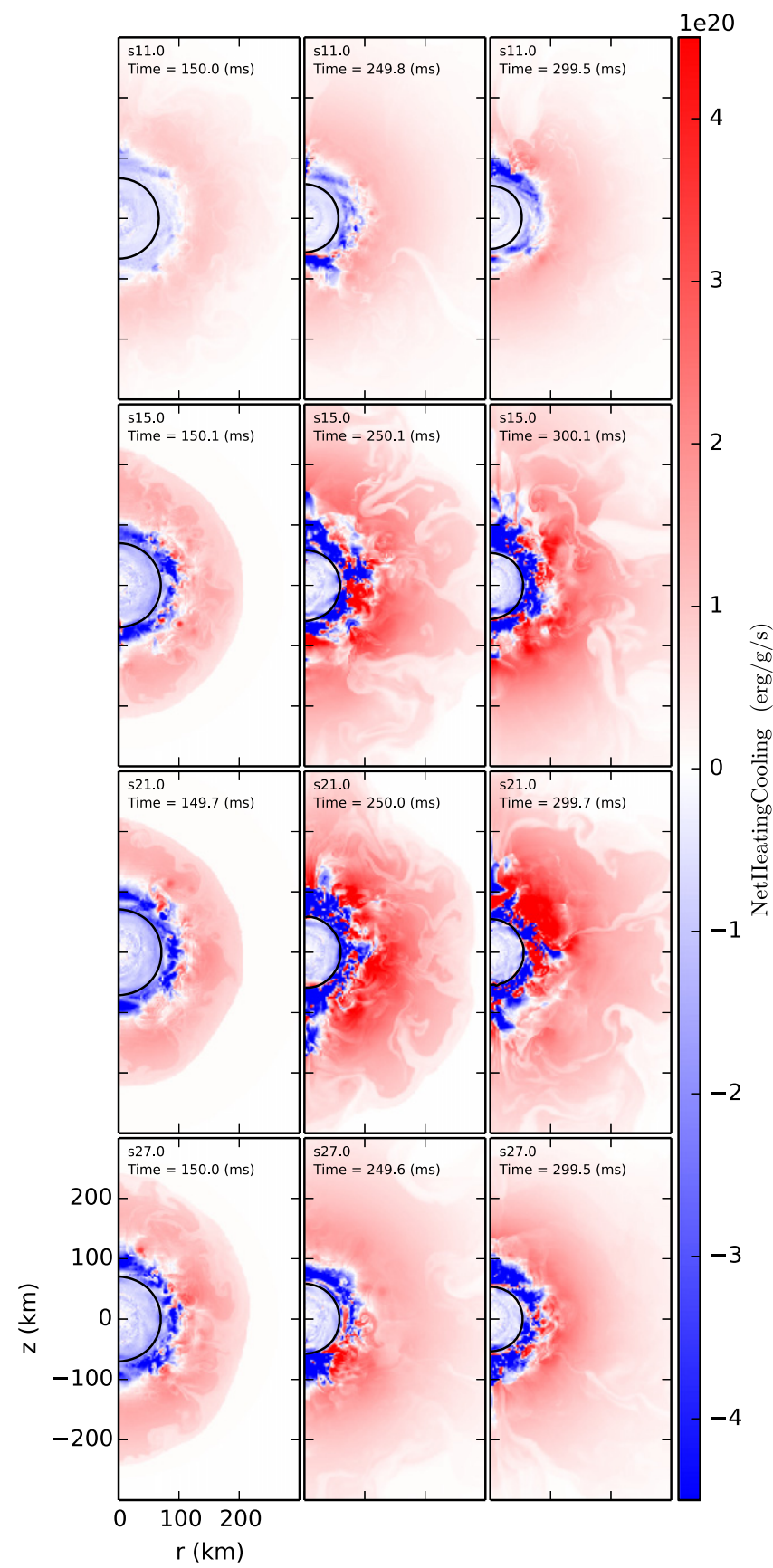

Figure 25. Net heating (red) and cooling (blue) distributions of our models 2DDP (see Table 4). The solid black lines show the radius of the PNS.

Figure 24 shows the neutrino luminosity and mean energy of electron-type neutrinos and antineutrinos as functions of time. The neutrino mean energy, $E_{\nu}$, is defined by $E_{\nu}=\frac{\int \epsilon^{3} f(\nu, \epsilon) d \epsilon}{\int \epsilon^{2} f(\nu, \epsilon) d \epsilon}$, where $f$ is the distribution function. The neutrino luminosities in models DA after $\sim 50 \mathrm{~ms}$ grow faster than in models DP but the peak luminosities after $\sim 100 \mathrm{~ms}$ are similar in both models DA and DP. The neutrino mean energies show a similar trend but the differences are less pronounced. We note that the neutrino luminosities and mean energies in our simulations are of a similar order of magnitude to the typical results in the literature (Janka 2012). Therefore, the high explosion energies in our simulations require a larger mass in the gain region or a higher heating efficiency than other investigators.
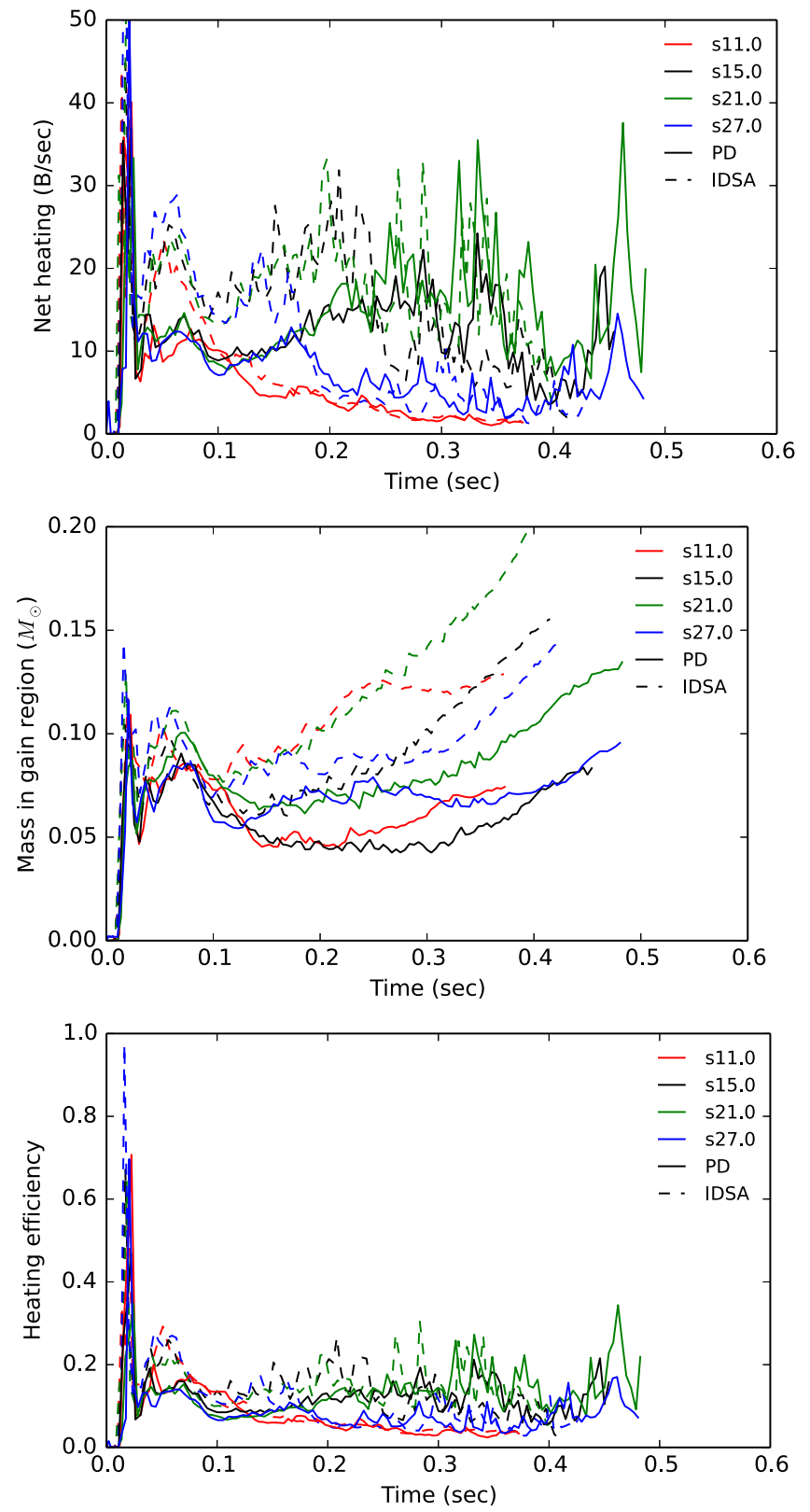

Figure 26. Integral quantities of net heating (top), mass in the gain region (middle), and heating efficiency (bottom) as functions of time. See Section 4 for the definition of these integral quantities. Different colors represent different progenitor models. Solid lines represent the models 2D-DP and dashed lines indicate the models 2D-DA in Table 4.

Figure 25 presents the $2 \mathrm{D}$ net heating and cooling rates in models 2D-DP. The red color shows the heating region and the blue color indicates the cooling region. The PNS radius (defined by the average radius of density $\rho=10^{11} \mathrm{~g} \mathrm{~cm}^{-3}$ ) is plotted as well in Figure 25. The gain region is defined as the post-shock region with positive net heating. In Figure 26, we show the integrated quantities of net heating $\left(Q_{\text {net }}\right)$, total mass in the gain region, and the heating efficiency as functions of time. The heating efficiency is defined by $\eta_{\text {heat }}=Q_{\text {net }} /\left(L_{\nu_{e} \text {,gain }}+L_{\bar{\nu}_{e} \text {,gain }}\right)$, since the heating originates mainly from the electron-type neutrinos.

The early prompt convection shows a very high peak in heating efficiency at $\sim 20 \mathrm{~ms}$, which could be associated with a grid-effect in cylindrical coordinates. However, this prompt 

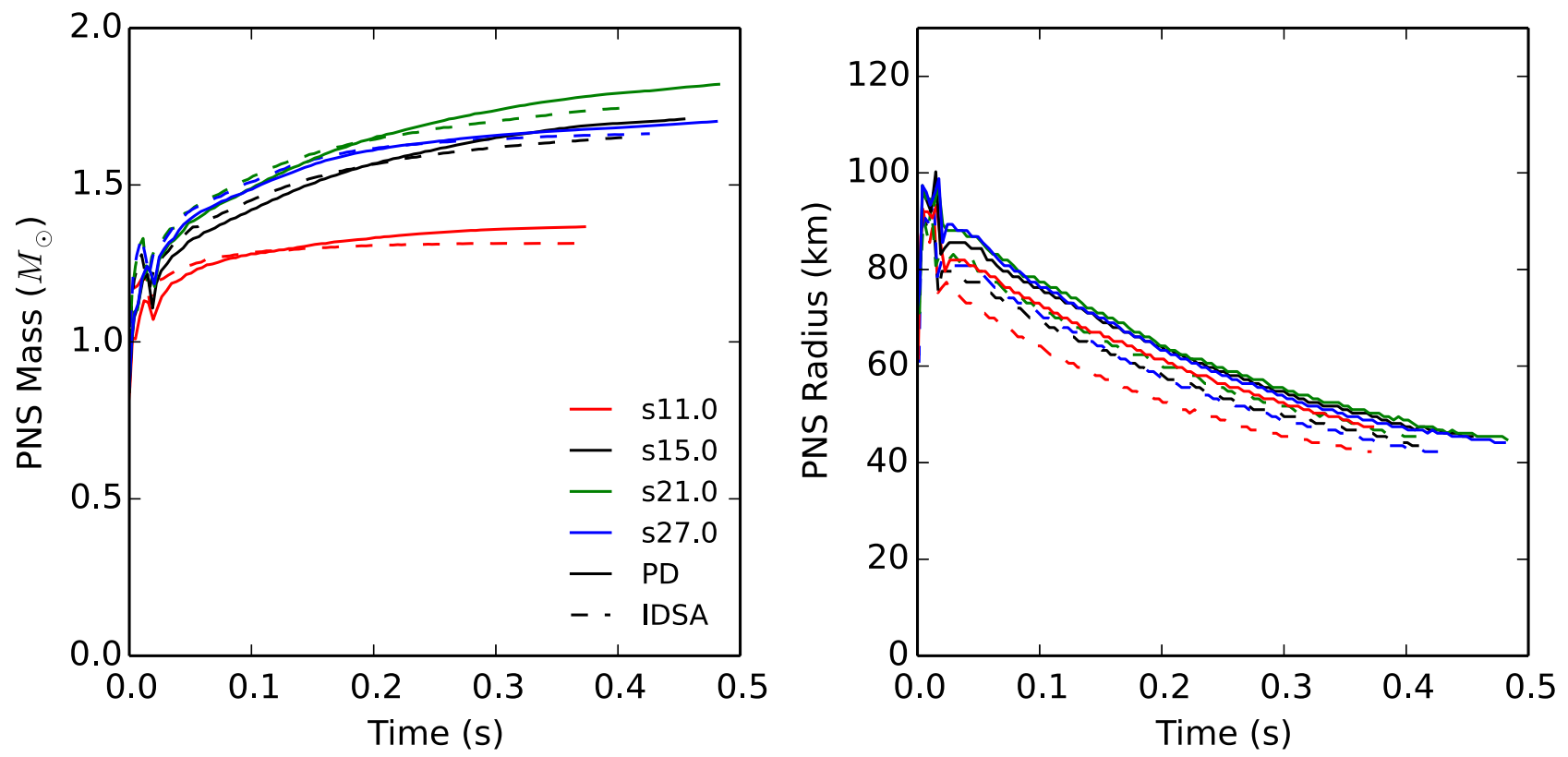

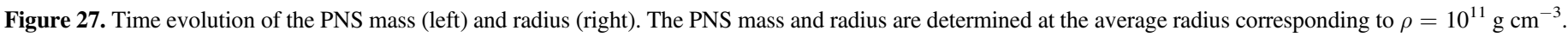
Different colors represent different progenitor stars. Solid lines show the models 2D-DP and dashed lines show the models $2 \mathrm{D}-\mathrm{DA}$ in Table 4.

heating does not affect the explosion energy directly since matter is still bound at that time. The shock expansion and the early convection at $t_{\mathrm{pb}} \sim 50 \mathrm{~ms}$ enlarge the gain radius and the mass in the gain region. After $t_{\mathrm{pb}} \sim 100-200 \mathrm{~ms}$, the mass in the gain region continues to increase with time. We noted that the masses in the gain region $\left(\sim 0.1 M_{\odot}\right)$ in our simulations are higher than the values that are reported by other groups, explaining the high diagnostic explosion energy in our simulations.

The models 2D-DA11 and 2D-DP11 have the lowest net heating and heating efficiency among the models (except during the prompt convection period), but have the second highest mass in the gain region and diagnostic energy. This is probably due to the fast shock expansion (see Figure 14) and low mass-accretion in the progenitor s11.0.

\subsection{Proto-neutron Stars}

During its evolution, the PNS shrinks due to the neutrino cooling behind the gain radius. In Figure 27, we show the evolutions of mass and radius of the PNSs after bounce. An initial oscillation of the PNS takes place at $\sim 10 \mathrm{~ms}$, when the shock breaks through the neutrino sphere. Later on, mass continuously accretes onto the PNS for several hundred milliseconds so that it reaches $\sim 1.3-2.0 M_{\odot}$ at the end of the simulations. At that time, the PNS radii have shrunken to $\sim 40 \mathrm{~km}$. The mass and radii of the PNSs at the end of the simulations are summarized in Table 4.

The convection and SASI activities help the PNS to accrete mass during the setup of an explosion. Accretion funnels are created along the high-entropy convective plumes. This can be seen in Figure 20. Therefore, the progenitor s11.0, which has the least SASI activities and accretion funnels, shows the lowest PNS mass increment, and the growth rate of the PNS mass is the lowest as well. Furthermore, the progenitor s11.0 has the lowest density distribution outside the iron core, which gives the lowest mass-accretion rate. After $\sim 400 \mathrm{~ms}$, the mass increment of the PNS becomes small but the mass of the PNS is still increasing at the end of the simulations. We find that although the growth rates of the PNS mass in models 2D-DA and 2D-DP are different, the final PNS masses in models 2DDP are only slightly larger $(<5 \%)$, and the radii of PNSs in models 2D-DA are slightly lower than those in models 2D-DP, giving a more compact PNS core in models 2D-DA. This can also be related to the earlier explosion times of models DA.

Figure 28 shows the central density, central electron fraction, lepton fraction, central entropy, and central temperature as functions of time. Models 2D-DA show higher central densities and higher central $Y_{e}$ (higher electron pressures), but lower central entropies and central temperatures than the models 2DDP, giving a more compact PNS core. However, the density in the outer layer of the PNS is higher and more convective in models 2D-DP than that in models 2D-DA. Therefore, models 2D-DP have a higher PNS mass and radius (see Figure 27).

\section{CONCLUSIONS}

We have performed self-consistent CCSN simulations for the four non-rotating, solar-metallicity progenitors, s11.0, s15.0, s21.0, and s27.0 from Woosley et al. (2002), and s15 and s20 from Woosley \& Heger (2007), using the AMR code FLASH with an IDSA for neutrino transport. A very good agreement of FLASH-IDSA with AGILE-IDSA is shown in Section 3.1, though some small differences still exist. In addition, we have provided a comparison of our multidimensional IDSA results for the s15 and s20 progenitors from Woosley \& Heger (2007) with results in the existing literature. However, a detailed comparison with an exchange of data and collaboration among groups would be necessary to understand remaining different results with regard to the underlying physics. 

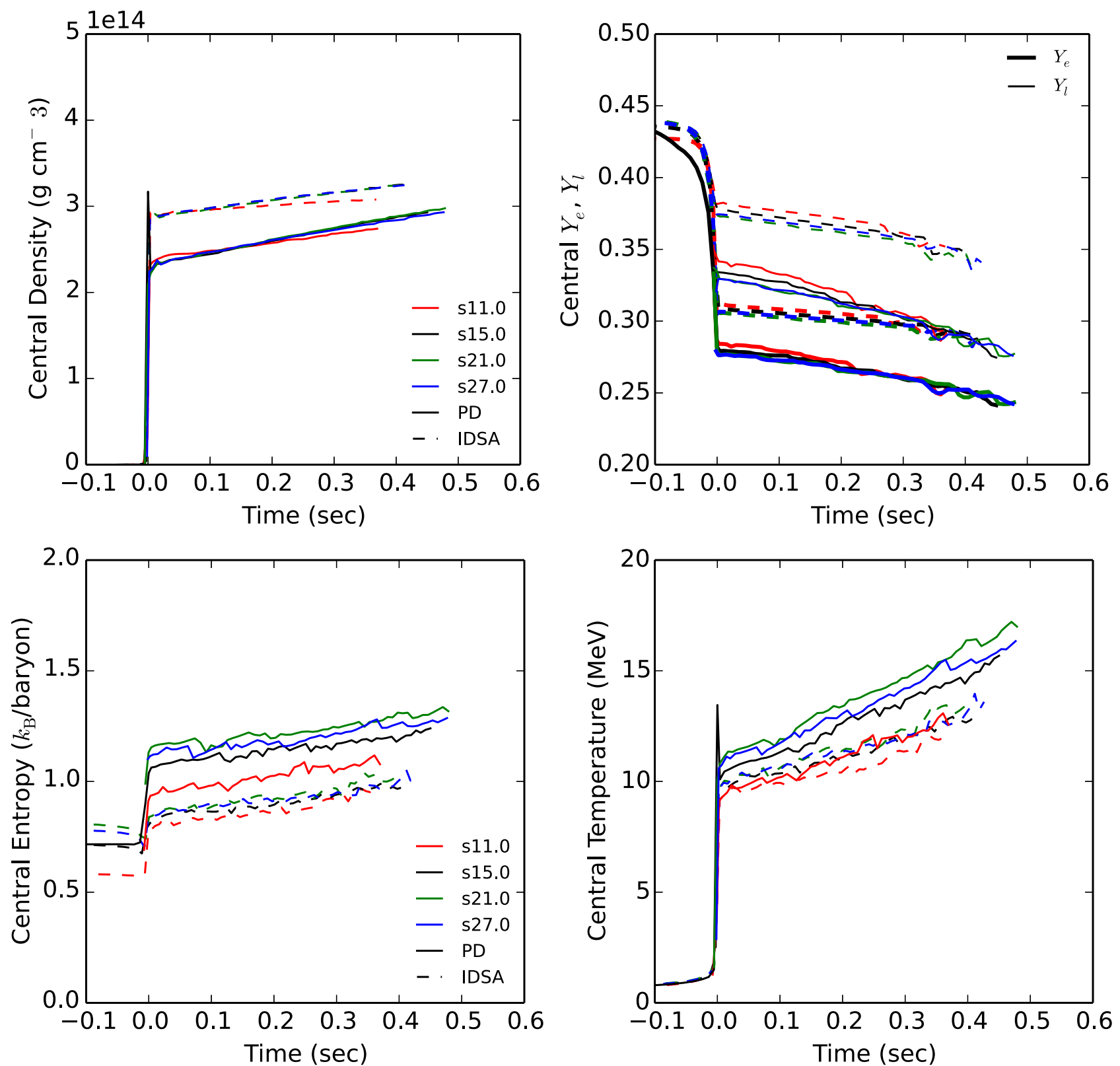

Figure 28. Time evolution of central density ( $\rho_{c}$, upper left), central electron fraction $\left(Y_{e}\right)$ and lepton fraction $\left(Y_{l}\right.$, upper right), central entropy (lower left), and central temperature (lower right). Different colors represent different progenitor stars. Solid lines show the models 2D-DP and dashed lines show the models 2D-DA in Table 4. In the upper right panel, the thin lines represent the central lepton fraction and the thick lines represent the central electron fraction.

The new SN EOS HS(DD2) is employed in this study and a comparison with the standard LS220 EOS is discussed in the Appendix. It is found that the DD2 EOS shows a faster shock expansion, and a higher mass in the gain region, while the neutrino luminosities are similar, causing the DD2 EOS to lead to slightly earlier and stronger explosions than the LS220.

We have presented two sets of simulations which compared the neutrino transport with the IDSA (but ignoring NES; abbreviated DA) with the PD scheme (which includes NES effectively; abbreviated DP) during collapse. The results show clearly that the treatment of neutrino weak interactions and the level of deleptonziation during the collapse phase have a significant impact on the neutrino-driven explosions. All our 2D models explode within $\sim 100-300 \mathrm{~ms}$. Models without NES explode much more easily, more strongly and faster than models with NES. The diagnostic explosion energy in our simulations is around $0.1-0.5 \mathrm{~B}$ in models 2D-DP (see Table 4) and around $0.2-0.8 \mathrm{~B}$ in models 2D-DA. Our explosion energies are likely to decrease when we include generalrelativistic effects and better electron-capture rates in future models.

In our simulations, we do not use the typical RbR approach for the neutrino transport. Instead, we solve the diffusion part in multiple dimensions, improving the neutrino transport in angular and temporal directions. With this approach, the prompt convection after the core bounce causes a fast shock expansion $\left(t_{\mathrm{pb}} \sim 50 \mathrm{~ms}\right)$, enlarging the gain region at late time, and therefore helps to increase the explosion energy at 

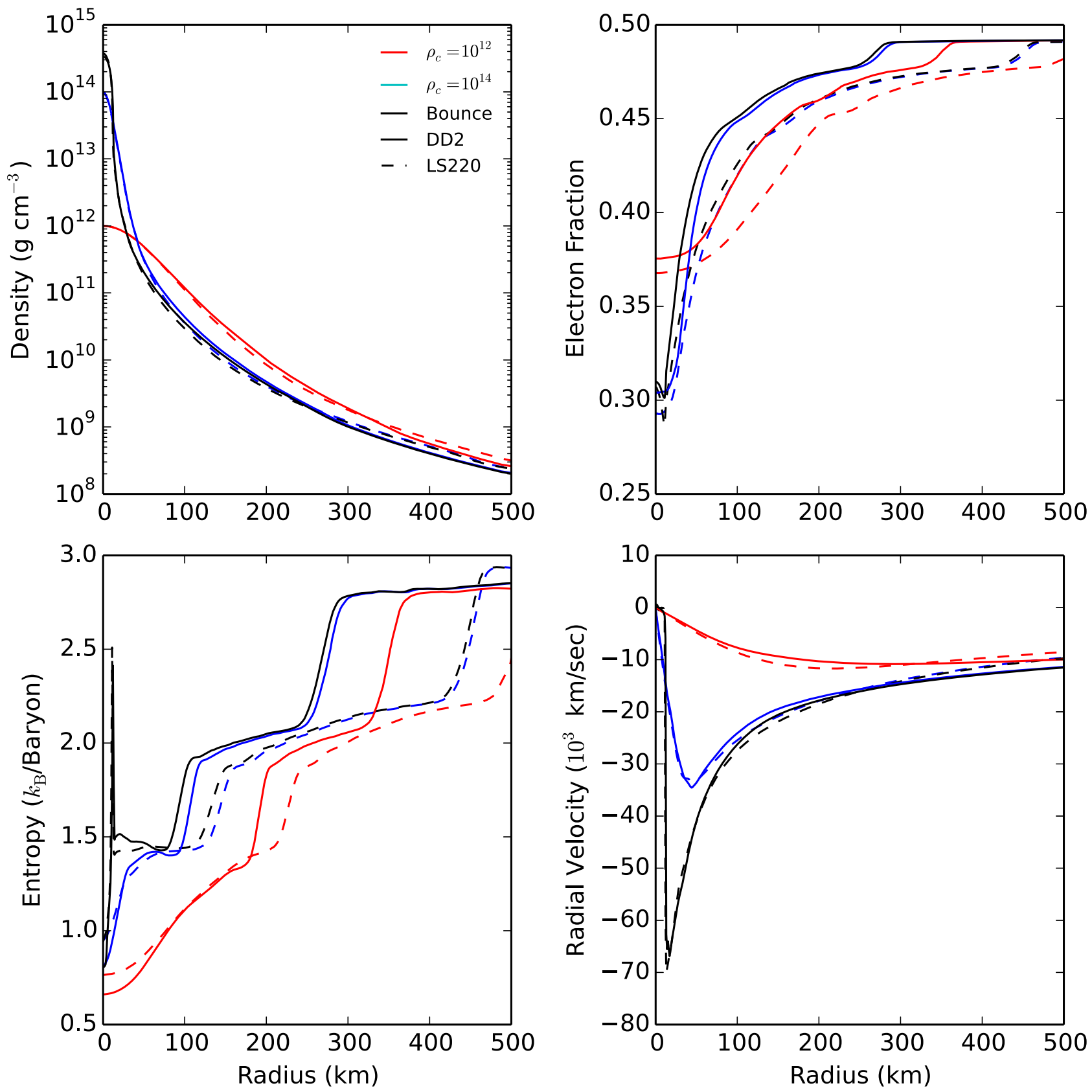

Figure 29. Similar to Figure 10, but for models 2D-DA15 and 2D-LA15 in Table 4.

late time. However, this prompt convection may be amplified by grid-effects in cylindrical coordinates. To address this issue, a full 3D simulation will be necessary or a detailed comparison with simulations in spherical coordinates. Furthermore, we have found SASI activities at late time in progenitors s15.0, s21.0, and s27.0, but in most cases the explosions are mainly due to neutrino-driven convection. Future work will relax the constraints imposed by axisymmetry. Models in 3D will permit the study of a more realistic turbulence cascade and rotation.

We thank the anonymous referee for valuable comments and suggestions. This work was supported by the European Research Council (ERC; FP7) under ERC Advanced grant agreement No. 321263-FISH, by the PASC High Performance Computing Grant DIAPHANE, and by the Swiss National Science Foundation (SNF). The Basel group is a member of the COST Action New Compstar. Part of this work was inspired during the MICRA2013 meeting in ECT*. We thank Albino Perego for the development of the leakage scheme for heavy neutrinos. We thank Takami Kuroda, Rubén Cabezón, Kei Kotake, Tomoya Takiwaki, Ko Nakamura, and Yudai Suwa for useful discussions. K.C.P. acknowledges Sean Couch for releasing his supernova setup in the public version of FLASH. FLASH was developed largely by the DOE-supported ASC/Alliances Center for Astrophysical Thermonuclear Flashes at the University of Chicago. The simulations have been carried out at the CSCS Monte-Rosa under grant No. 412. 

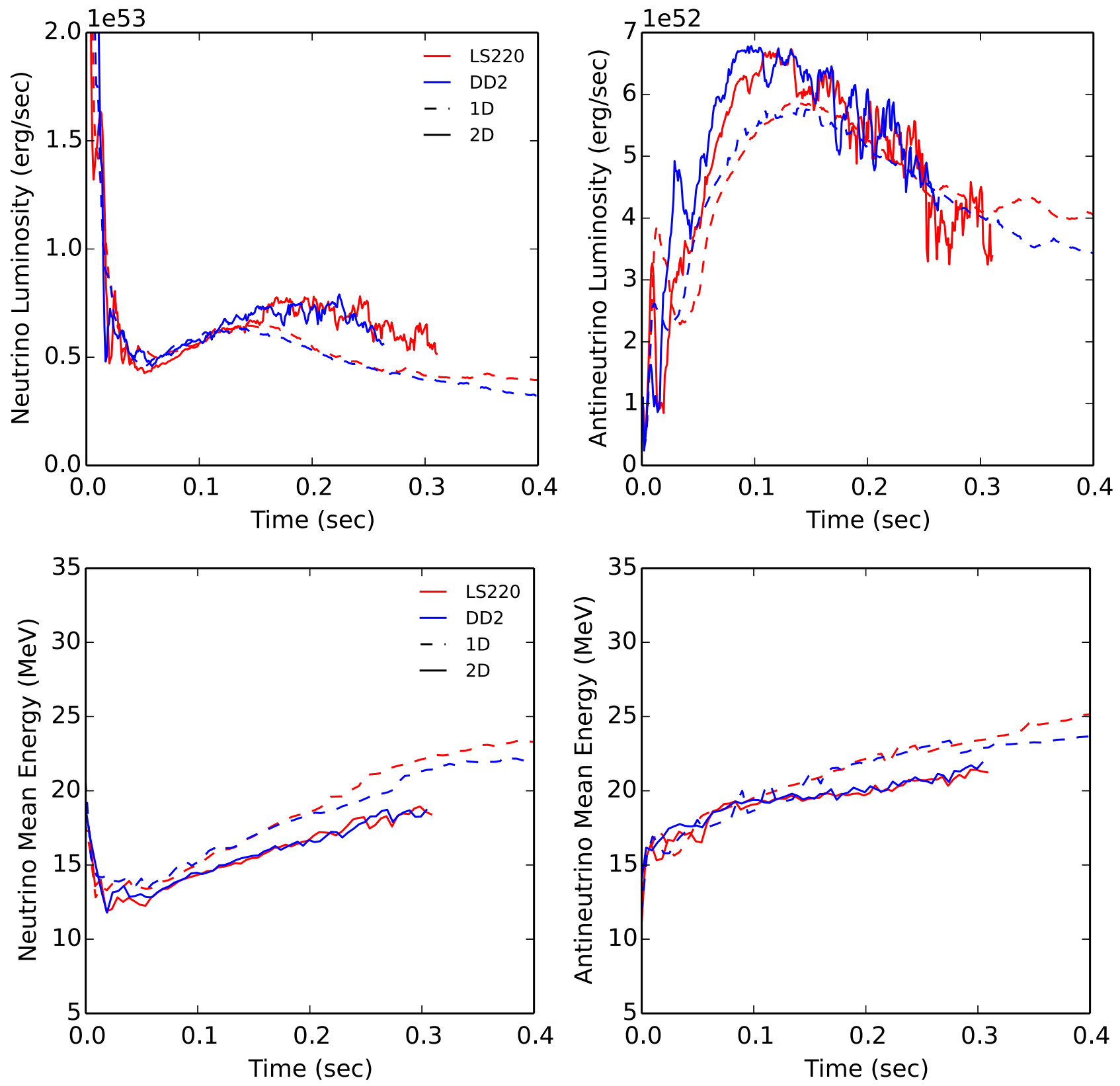

Figure 30. Similar to Figure 24, but for models 1D-DA15, 1D-LA15, 2D-DA15, and 2D-LA15 in Table 4.

Analysis and visualization of simulation data were completed using the analysis toolkit yt (Turk et al. 2011).

\section{APPENDIX \\ A COMPARSION BETWEEN DD2 AND LS220}

Several simulations in this paper use the new DD2 EOS, while most multidimensional simulations found in the literatures use LS220. We therefore briefly describe the qualitative differences between the simulations with the DD2 and the LS220 EOSs in this section. We perform 1D and 2D simulations of the progenitor s15.0 with DD2 and LS220 EOSs (models 1D-DA15, 2D-DA15, 1D-LA15, and 2D-LA15 in Table 4). Hereafter, we use DA15 to refer to both models 1DDA15 and 2D-DA15, and similarly we use LA15 to refer to the models 1D-LA15 and 2D-LA15. Their main features are summarized in Table 4. The PD scheme is turned off in order to study the impact of the EOS on the neutrino transport.

The angle-averaged radial profiles of density, electron fraction, entropy, and radial velocity are shown in Figure 29 for the prebounce phase. As discussed in Section 4, the radial profiles in the collapse phase are nearly identical in both 1D and 2D. In addition, the density and radial velocity evolutions in models DA15 and LA15 are also similar during the collapse but models DA15 collapse more slowly than models LA15. Therefore, models DA15 reach core bounce about $\sim 30 \mathrm{~ms}$ later than models LA15.

While there are no significant differences in the neutrino luminosity and mean energy between models DA15 and LA15 (Figure 30), models DA15 show a lower central density and higher PNS radius than LA15. The time evolutions of central density and electron fraction of models DA15 and LA15 are 

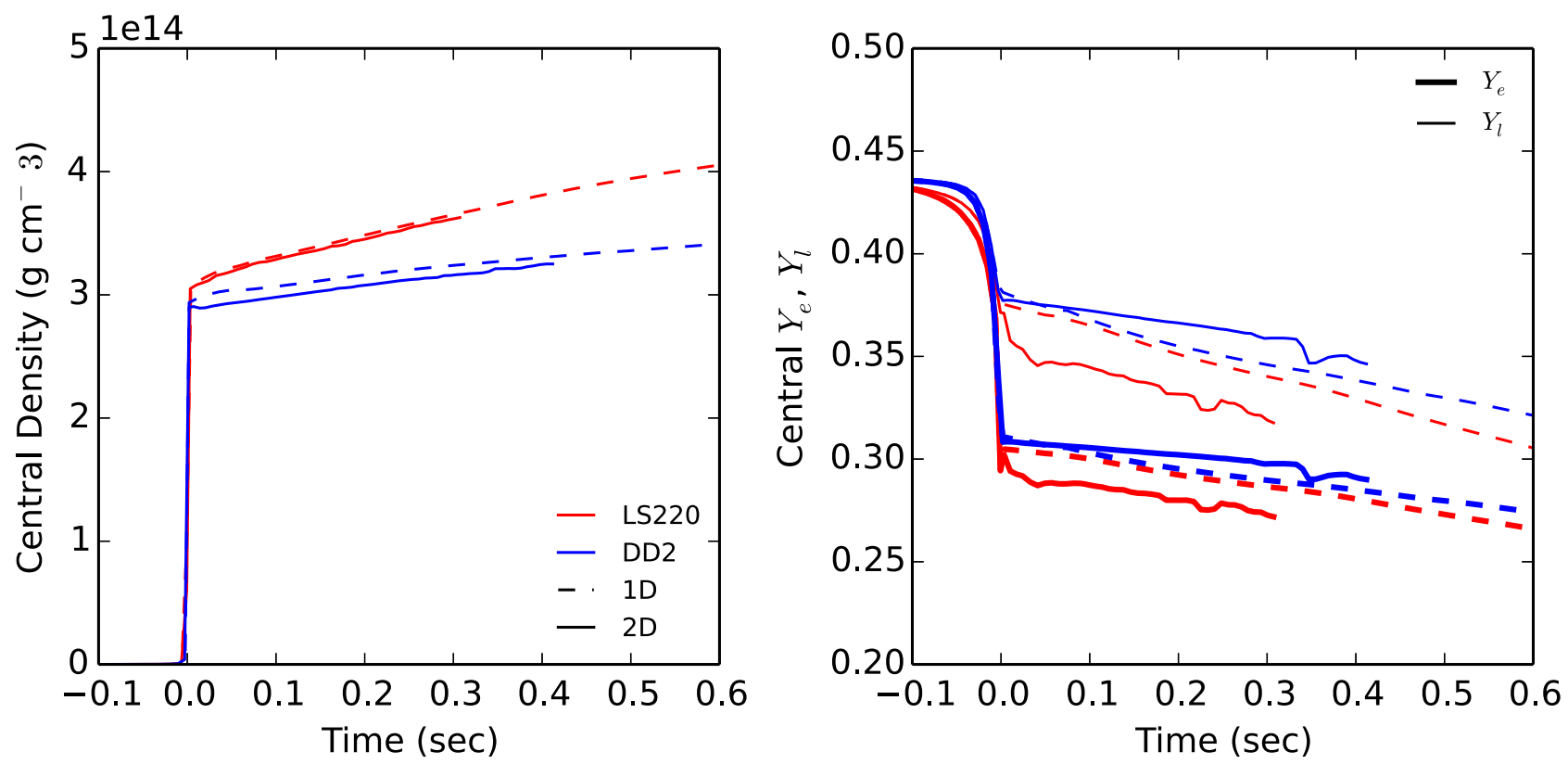

Figure 31. Similar to Figure 28, but for models 1D-DA15, 1D-LA15, 2D-DA15, and 2D-LA15 in Table 4.
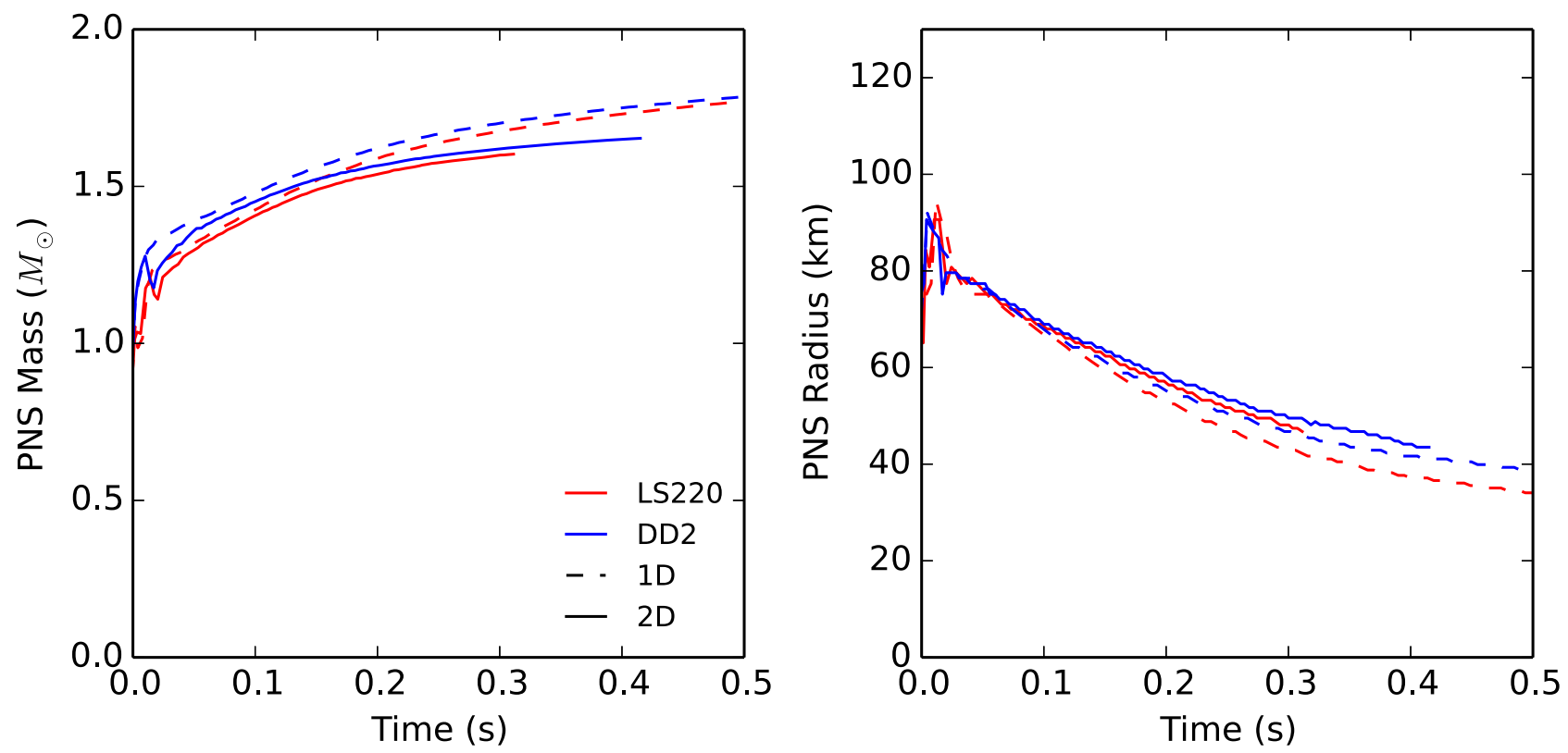

Figure 32. Similar to Figure 27, but for models 2D-DA15 and 2D-LA15 in Table 4.

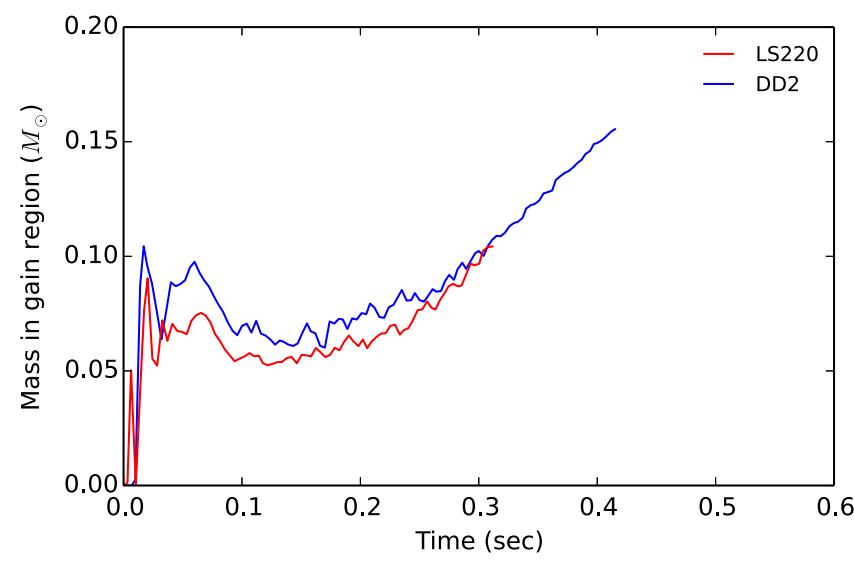

Figure 33. Similar to Figure 26, but for models 2D-DA15 and 2D-LA15 in Table 4.

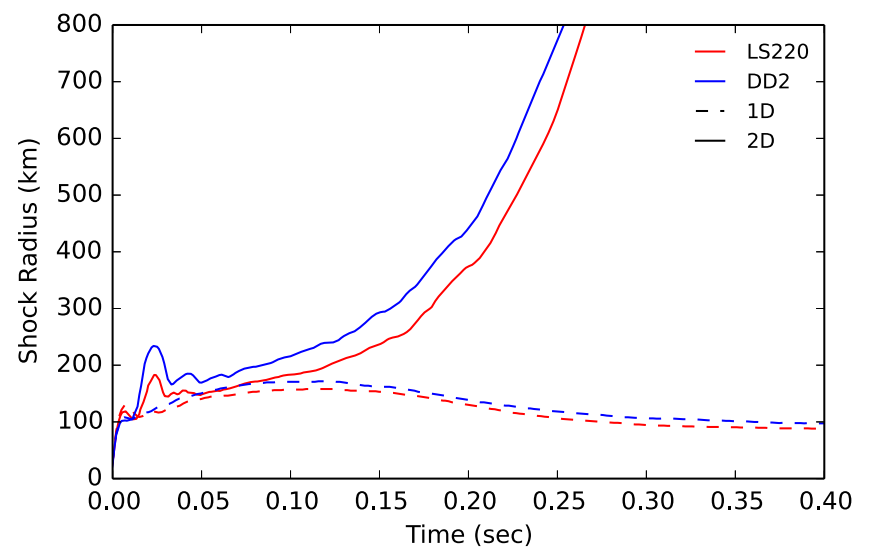

Figure 34. Similar to Figure 14, but for models 1D-DA15, 1D-LA15, 2DDA15, and 2D-LA15 in Table 4. 

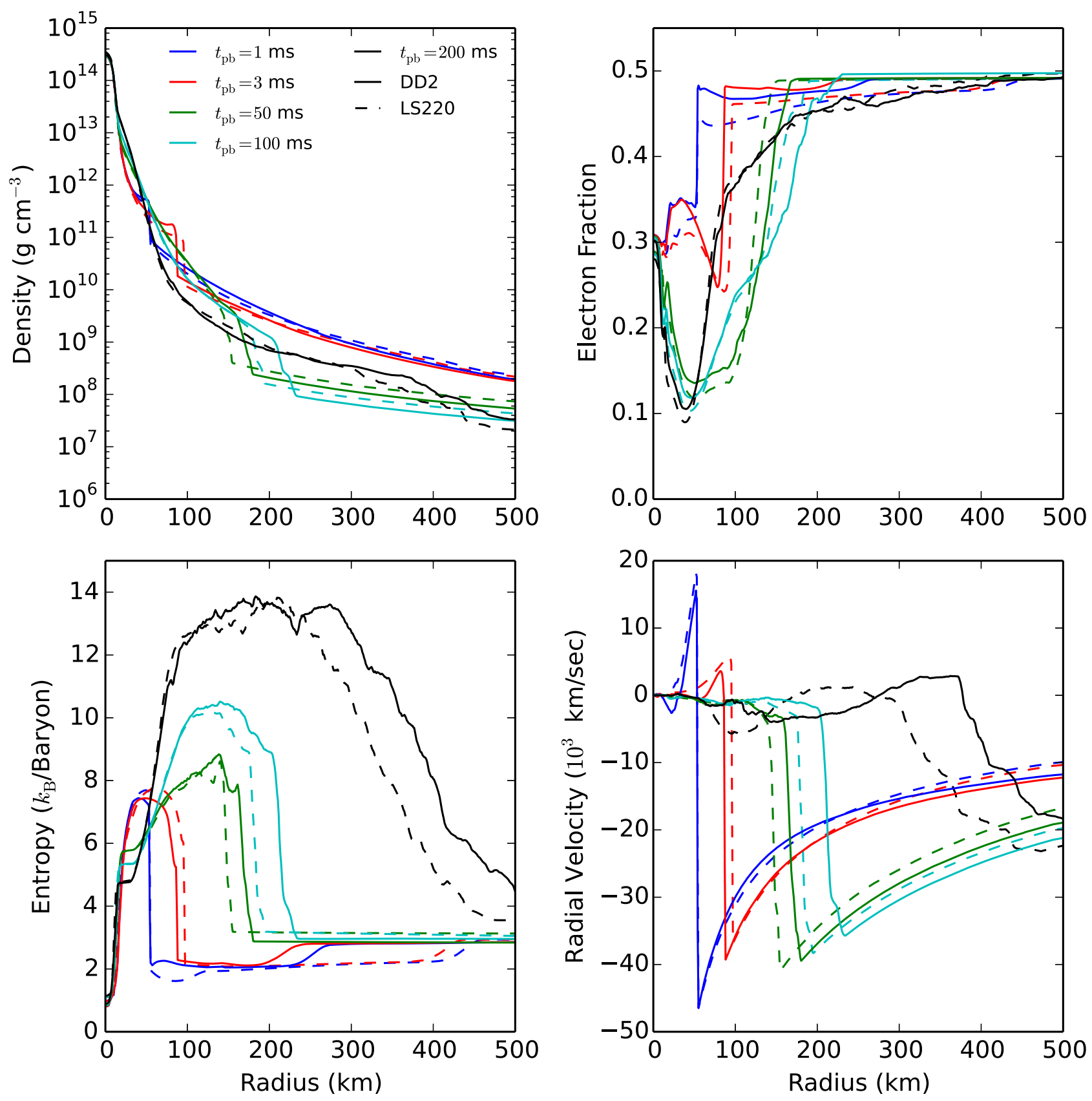

Figure 35. Similar to Figure 16, but for models 1D-DA15, 1D-LA15, 2D-DA15, and 2D-LA15 in Table 4.

shown in Figure 31. Furthermore, models DA15 have a higher central electron fraction and lepton fraction than models DL15. 2D models also show a lower PNS mass and a higher PNS radius than 1D (Figure 32), but the PNS masses are similar in both DD2 and LS220 (see Table 4).

Since the bounce time in models DA15 is longer than in models LA15, the $\mathrm{Si} / \mathrm{O}$ interface reaches a smaller radius in models DA15 $(\sim 90 \mathrm{~km})$ than in LA15 $(\sim 120 \mathrm{~km}$, see Figure 29). The bounce shock hits the $\mathrm{Si} / \mathrm{O}$ interface earlier in models DA15 than in LA15, generating a prompt convection and fast shock expansion at $\sim 20 \mathrm{~ms}$ postbounce. The earlier interaction between the bounce shock and the $\mathrm{Si} / \mathrm{O}$ interface in model 2D-DA15 makes the shock radius in model 2D-DA15 larger than in simulation 2D-LA15. Furthermore, the larger shock radius in model 2 D-DA15 produces a higher mass in the gain region (see Figure 33), suggesting that the models with the DD2 EOS explode more easily than the models with the LS220 EOS. The evolution of averaged shock radius of models DA15 and LA15 can be seen in Figure 34. The explosion time $\left(t_{400}\right)$ in 2D-DA15 is about $23 \mathrm{~ms}$ earlier than in 2D-LA15. In Figure 35, we compare the radial density, electron fraction, entropy, and velocity profiles in 1D and 2D for DD2 and LS220 at 1, 3, 50, 100, and $200 \mathrm{~ms}$ postbounce. Before $\sim 20 \mathrm{~ms}$ postbounce, models LS15 show a faster shock expansion than models DA15 but the shock radius in DA15 become larger after the $\mathrm{Si} / \mathrm{O}$ interface has reached the shock at $\sim 20 \mathrm{~ms}$.

Figure 36 shows the normalized decomposition into Legendre polynomials (Equation (15)) of the shock radius of models 2D-DA15 and 2D-LA15. The corresponding entropy 

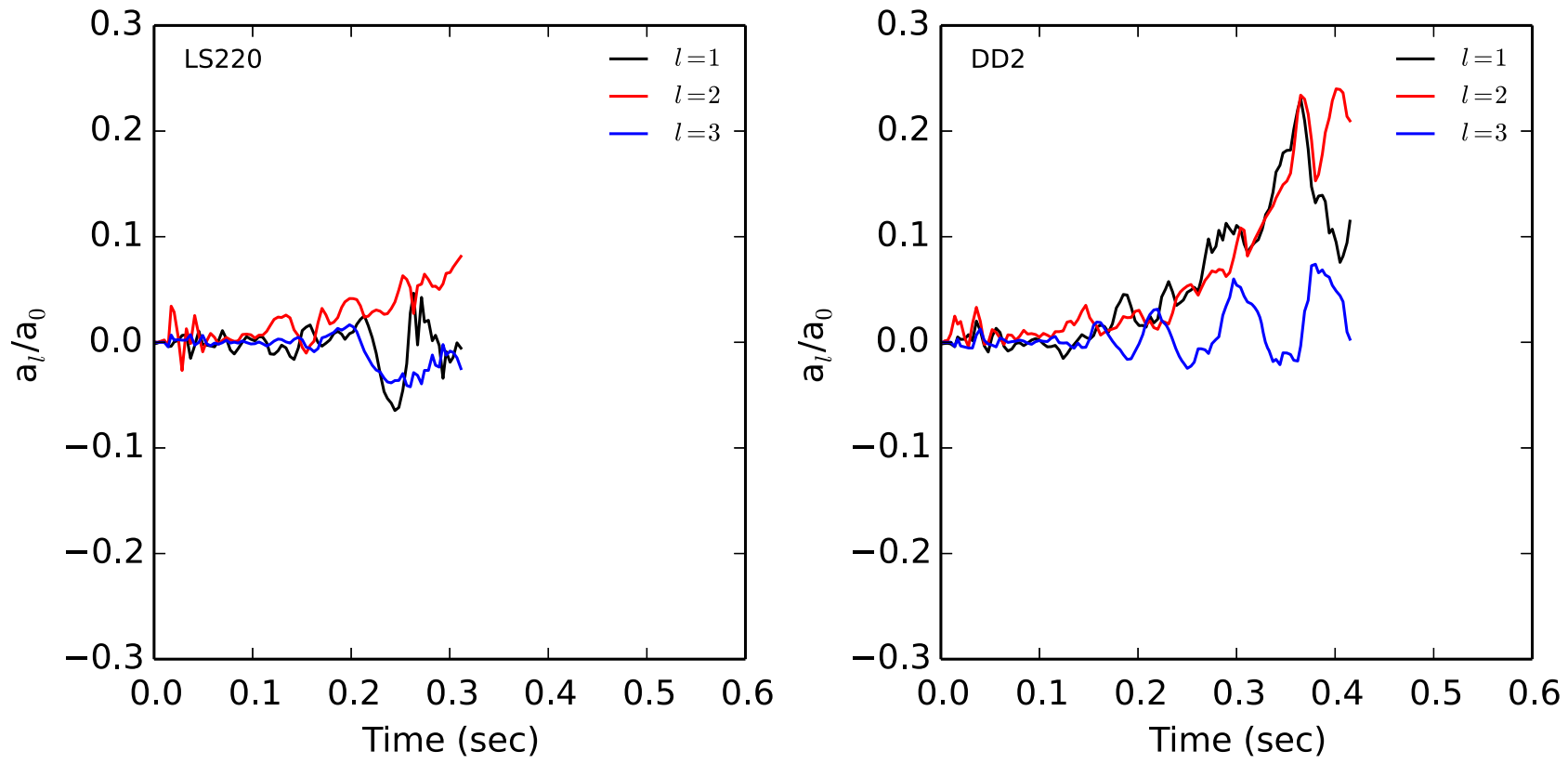

Figure 36. Similar to Figure 21, but for models 2D-LA15 (left) and 2D-LD15 (right) in Table 4.
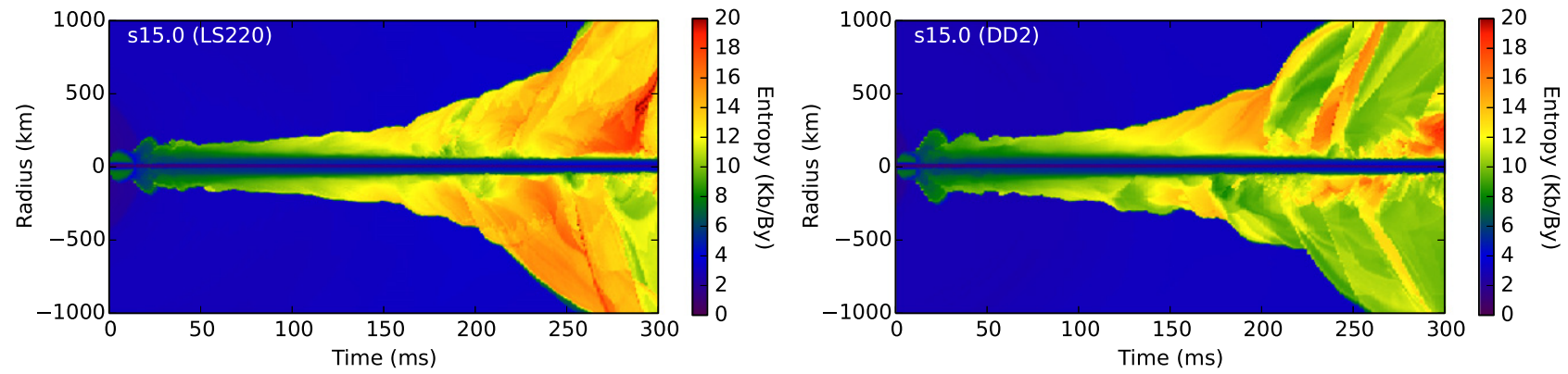

Figure 37. Similar to Figure 22, but for models 2D-LA15 (left) and 2D-DA15 (right) in Table 4.

distribution at the north and south poles is shown in Figure 37. The SASI activities start at $\sim 0.2 \mathrm{~s}$ in both 2D-DA15 and 2DLA15 after the $\mathrm{Si} / \mathrm{O}$ interface reaches the shock, and the amplitudes of the normalized coefficients $a_{l} / a_{0}$ do not show significant difference. We remark that a more through investigation of the EOS effects will be the subject of a future study.

\section{REFERENCES}

Berninger, H., Frenod, E., Gander, M., Liebendorfer, M., \& Michaud, J. 2013, SJMA, 45, 3229

Bethe, H. A. 1990, RvMP, 62, 801

Bethe, H. A., \& Pizzochero, P. 1990, ApJL, 350, L33

Blondin, J. M., Mezzacappa, A., \& DeMarino, C. 2003, ApJ, 584, 971

Brandt, T. D., Burrows, A., Ott, C. D., \& Livne, E. 2011, ApJ, 728, 8

Bruenn, S. W. 1985, ApJS, 58, 771

Bruenn, S. W. 1989a, ApJ, 340, 955

Bruenn, S. W. 1989b, ApJ, 341, 385

Bruenn, S. W., De Nisco, K. R., \& Mezzacappa, A. 2001, ApJ, 560, 326

Bruenn, S. W., Lentz, E. J., Hix, W. R., et al. 2014, arXiv:1409.5779

Bruenn, S. W., Mezzacappa, A., Hix, W. R., et al. 2013, ApJL, 767, L6

Buras, R., Rampp, M., Janka, H.-T., \& Kifonidis, K. 2006, A\&A, 447, 1049

Burrows, A. 2013, RvMP, 85, 245

Burrows, A., Young, T., Pinto, P., Eastman, R., \& Thompson, T. A. 2000, ApJ, 539,865

Colella, P., \& Woodward, P. R. 1984, JCoPh, 54, 174
Colgate, S. A., \& White, R. H. 1966, ApJ, 143, 626

Couch, S. M. 2013, ApJ, 765, 29

Couch, S. M., Chatzopoulos, E., Arnett, W. D., \& Timmes, F. X. 2015, ApJL, 808, L21

Couch, S. M., Graziani, C., \& Flocke, N. 2013, ApJ, 778, 181

Couch, S. M., \& O'Connor, E. P. 2014, ApJ, 785, 123

Couch, S. M., \& Ott, C. D. 2013, ApJL, 778, L7

Dolence, J. C., Burrows, A., \& Zhang, W. 2015, ApJ, 800, 10

Dubey, A., Reid, L. B., \& Fisher, R. 2008, PhST, 132, 014046

Fischer, T., Hempel, M., Sagert, I., Suwa, Y., \& Schaffner-Bielich, J. 2014, EPJA, 50, 46

Fischer, T., Martínez-Pinedo, G., Hempel, M., \& Liebendörfer, M. 2012, PhRvD, 85, 083003

Foglizzo, T., Kazeroni, R., Guilet, J., et al. 2015, PASA, 32, 009

Fryer, C., Benz, W., Herant, M., \& Colgate, S. A. 1999, ApJ, 516, 892

Fryxell, B., Olson, K., Ricker, P., et al. 2000, ApJS, 131, 273

Hanke, F. 2014, PhD thesis, Technische Universität München

Hanke, F., Marek, A., Müller, B., \& Janka, H.-T. 2012, ApJ, 755, 138

Hannestad, S., \& Raffelt, G. 1998, ApJ, 507, 339

Hempel, M. 2014, arXiv:1410.6337

Hempel, M. 2015, PhRvC, 91, 055807

Hempel, M., Hagel, K., Natowitz, J., Röpke, G., \& Typel, S. 2015, PhRvC, 91, 045805

Hempel, M., \& Schaffner-Bielich, J. 2010, NuPhA, 837, 210

Hix, W. R., Messer, O. E., Mezzacappa, A., et al. 2003, PhRvL, 91, 201102 Janka, H.-T. 2012, ARNPS, 62, 407

Janka, H.-T., \& Mueller, E. 1996, A\&A, 306, 167

Krüger, T., Tews, I., Hebeler, K., \& Schwenk, A. 2013, PhRvC, 88, 025802

Kuroda, T., Kotake, K., \& Takiwaki, T. 2012, ApJ, 755, 11 
Kuroda, T., Takiwaki, T., \& Kotake, K. 2015, arXiv:1501.06330

Langanke, K., Martínez-Pinedo, G., Sampaio, J. M., et al. 2003, PhRvL, 90, 241102

Lattimer, J. M., \& Swesty, F. D. 1991, NuPhA, 535, 331

Ledoux, P. 1947, ApJ, 105, 305

Lee, D. 2013, JCoPh, 243, 269

Lentz, E. J., Mezzacappa, A., Messer, O. E. B., Hix, W. R., \& Bruenn, S. W. 2012a, ApJ, 760, 94

Lentz, E. J., Mezzacappa, A., Messer, O. E. B., et al. 2012b, ApJ, 747, 73

Liebendörfer, M. 2005, ApJ, 633, 1042

Liebendörfer, M., Messer, O. E. B., Mezzacappa, A., et al. 2004, ApJS, 150,263

Liebendörfer, M., Rampp, M., Janka, H.-T., \& Mezzacappa, A. 2005, ApJ, 620,840

Liebendörfer, M., Whitehouse, S. C., \& Fischer, T. 2009, ApJ, 698, 1174

Livne, E., Burrows, A., Walder, R., Lichtenstadt, I., \& Thompson, T. A. 2004, ApJ, 609, 277

Melson, T., Janka, H.-T., Bollig, R., et al. 2015a, ApJL, 808, L42

Melson, T., Janka, H.-T., \& Marek, A. 2015b, ApJL, 801, L24

Mezzacappa, A., \& Bruenn, S. W. 1993a, ApJ, 405, 669

Mezzacappa, A., \& Bruenn, S. W. 1993b, ApJ, 410, 740

Mueller, B., \& Janka, H.-T. 2014, arXiv:1409.4783

Müller, B., \& Janka, H.-T. 2015, MNRAS, 448, 2141

Müller, B., Janka, H.-T., \& Heger, A. 2012a, ApJ, 761, 72

Müller, B., Janka, H.-T., \& Marek, A. 2012b, ApJ, 756, 84

Murphy, J. W., \& Burrows, A. 2008, ApJ, 688, 1159

Nakamura, K., Takiwaki, T., Kuroda, T., \& Kotake, K. 2014, arXiv:1406.2415

Nomoto, K., \& Hashimoto, M. 1988, PhR, 163, 13

Obergaulinger, M., Janka, H.-T., \& Aloy, M. A. 2014, MNRAS, 445, 3169

O'Connor, E. 2014, arXiv:1411.7058

O’Connor, E. 2015, ApJS, 219, 24

O’Connor, E., \& Ott, C. D. 2010, CQGra, 27, 114103
O'Connor, E., \& Ott, C. D. 2011, ApJ, 730, 70

O'Connor, E., \& Ott, C. D. 2013, ApJ, 762, 126

Ott, C. D., Abdikamalov, E., Mösta, P., et al. 2013, ApJ, 768, 115

Ott, C. D., Burrows, A., Dessart, L., \& Livne, E. 2008, ApJ, 685, 1069

Perego, A., Hempel, M., Fröhlich, C., et al. 2015, ApJ, 806, 275

Perego, A., Rosswog, S., Cabezón, R. M., et al. 2014, MNRAS, 443, 3134

Pons, J. A., Ibáñez, J. M., \& Miralles, J. A. 2000, MNRAS, 317, 550

Quirk, J. J. 1991, PhD thesis

Radice, D., Couch, S. M., \& Ott, C. D. 2015, ComAC, 2, 7

Rampp, M., \& Janka, H.-T. 2002, A\&A, 396, 361

Rosswog, S., \& Liebendörfer, M. 2003, MNRAS, 342, 673

Scheck, L., Kifonidis, K., Janka, H.-T., \& Müller, E. 2006, A\&A, 457, 963

Sumiyoshi, K., Takiwaki, T., Matsufuru, H., \& Yamada, S. 2015, ApJS, 216,5

Sumiyoshi, K., Yamada, S., Suzuki, H., et al. 2005, ApJ, 629, 922

Summa, A., Hanke, F., Janka, H.-T., et al. 2015, arXiv:1511.07871

Suwa, Y., Kotake, K., Takiwaki, T., Liebendörfer, M., \& Sato, K. 2011, ApJ, 738,165

Suwa, Y., Takiwaki, T., Kotake, K., et al. 2013, ApJ, 764, 99

Suwa, Y., Yamada, S., Takiwaki, T., \& Kotake, K. 2014, arXiv:1406.6414

Takiwaki, T., Kotake, K., \& Suwa, Y. 2012, ApJ, 749, 98

Takiwaki, T., Kotake, K., \& Suwa, Y. 2014, ApJ, 786, 83

Thompson, T. A., Burrows, A., \& Pinto, P. A. 2003, ApJ, 592, 434

Turk, M. J., Smith, B. D., Oishi, J. S., et al. 2011, ApJS, 192, 9

Typel, S., Röpke, G., Klähn, T., Blaschke, D., \& Wolter, H. H. 2010, PhRvC, 81,015803

Ugliano, M., Janka, H.-T., Marek, A., \& Arcones, A. 2012, ApJ, 757, 69

Woosley, S. E., \& Heger, A. 2007, PhR, 442, 269

Woosley, S. E., Heger, A., \& Weaver, T. A. 2002, RvMP, 74, 1015

Woosley, S. E., \& Weaver, T. A. 1995, ApJS, 101, 181

Yahil, A., \& Lattimer, J. M. 1982, in NATO Advanced Science Institutes (ASI) Series C, ed. M. J. Rees \& R. J. Stoneham (Berlin: Springer), 53 